\title{
Bat parasites (Acari, Anoplura, Cestoda, Diptera, Hemiptera, Nematoda, Siphonaptera, Trematoda) in France (1762-2018): a literature review and contribution to a checklist
}

\author{
Clément Léger*
}

Muséum national d'Histoire naturelle, Direction Générale Déléguée aux Collections (DGD-C) - Service Recherche, Enseignement, Expertise, CP 20, 38 rue Geoffroy Saint-Hilaire, 75005 Paris, France

Received 27 February 2020, Accepted 7 October 2020, Published online 18 November 2020

\begin{abstract}
This paper is a bibliographical survey of records of bat parasites in France (including Corsica) between 1762 and 2018. In total, 237 scientific publications were analysed. They show that bats are infected with a large diversity of endoparasites and ectoparasites. A total of 113 parasite taxa were identified from 27 host species; in addition, six bats were not identified to the species-level. The helminth fauna of bats comprises three species of Cestoda, 15 of Trematoda, and 13 of Nematoda. Acari parasites include 53 species (in addition to 22 invalid species). Finally, insect parasites comprise 13 species of Diptera (bat flies), 12 of Siphonaptera (fleas), 3 of Hemiptera (bugs), and 1 Anoplura species. Bat taxa reported with parasites were Barbastella barbastellus, Eptesicus serotinus, Hypsugo savii, Miniopterus schreibersii, Myotis bechsteinii, M. blythii, M. capaccinii, M. dasycneme, M. daubentonii, M. emarginatus, M. myotis, M. mystacinus, M. nattereri, M. punicus, Nyctalus lasiopterus, N. leisleri, N. noctula, Pipistrellus kuhlii, P. nathusii, P. pipistrellus, Plecotus auritus, P. austriacus, Rhinolophus euryale, R. ferrumequinum, R. hipposideros, R. mehelyi, Tadarida teniotis, Eptesicus sp., Myotis sp., Pipistrellus sp., Plecotus sp., Rhinolophus sp. and the species complex Pipistrellus pipistrellus/kuhlii/nathusii. As regards E. nilssonii, Vespertilio murinus (Particoloured Bat), M. alcathoe, M. escalerai, P. macrobullaris and P. pygmaeus, no records were found. These published field data originated from 72 of the 96 departments in metropolitan France. The most commonly cited were Ardèche, Ariège, Bouches-du-Rhône, Haute-Savoie, Maine-et-Loire, Moselle, Meurthe-et-Moselle, PyrénéesOrientales, Sarthe, Haute-Corse and Corse-du-Sud.
\end{abstract}

Key words: Bibliometry, Chiroptera, Host-parasite associations, Parasite biodiversity.

Résumé - Les parasites des Chiroptères (Acari, Anoplura, Cestoda, Diptera, Hemiptera, Nematoda, Siphonaptera, Trematoda) en France (1762-2018) - état des lieux bibliographique et contribution à une liste des espèces. Cet article est une étude bibliographique des signalements de parasites de chauves-souris en France (y compris la Corse) entre 1762 et 2018. Au total, 237 publications scientifiques ont été analysées. Elles montrent que les chauves-souris sont infectées par une grande diversité d'endoparasites et d'ectoparasites. Un total de 113 taxons parasites ont été identifiés parmi 27 espèces hôtes (en outre, six chauves-souris n'ont pas été identifiées au niveau de l'espèce). La faune helminthique des chauves-souris comprend trois espèces de Cestodes, 15 de Trématodes et 13 de Nématodes. Les Acariens parasites comprennent 53 espèces (en plus de 22 espèces invalides). Enfin, les insectes parasites comprennent 13 espèces de Diptères, 12 de Siphonaptères (puces), 3 d'Hémiptères (punaises) et 1 espèce d'Anoploure. Les taxons de chauves-souris signalés avec des parasites étaient Barbastella barbastellus, Eptesicus serotinus, Hypsugo savii, Miniopterus schreibersii, Myotis bechsteinii, M. blythii, M. capaccinii, M. dasycneme, M. daubentonii, M. emarginatus, M. myotis, M. mystacinus, M. nattereri, M. punicus, Nyctalus lasiopterus, N. leisleri, N. noctula, Pipistrellus kuhlii, P. nathusii, P. pipistrellus, Plecotus auritus, P. austriacus, Rhinolophus euryale, $R$. ferrumequinum, R. hipposideros, $R$. mehelyi, Tadarida teniotis, Eptesicus sp., Myotis sp., Pipistrellus sp., Plecotus sp., Rhinolophus sp. et le complexe d'espèces cryptiques Pipistrellus pipistrellus/ kuhlii/nathusii. Aucun signalement n'a été trouvé pour E. nilssonii, Vespertilio murinus, M. alcathoe, M. escalerai, $P$. macrobullaris et $P$. pygmaeus. Ces données de terrain publiées proviennent de 72 des 96 départements de France métropolitaine. Les plus cités sont l'Ardèche, l'Ariège, les Bouches-du-Rhône, la Haute-Savoie, le Maineet-Loire, la Moselle, la Meurthe-et-Moselle, les Pyrénées-Orientales, la Sarthe, la Haute-Corse et la Corse-du-Sud.

\footnotetext{
*Corresponding author: clement. leger@mnhn. fr
} 


\section{Introduction}

Bats (Mammalia: Chiroptera) represent the second-most diverse order of mammals, after rodents. As of 2007, 42 bat species have been reported from Europe (Dietz et al. [84]). According to Arthur \& Lemaire [16], 35 species have been unambiguously identified in France. Many aspects of the ecology of bats are under study (e.g. swarming, hunting sites, flight routes, habitat studies, acoustic ecology). One of these aspects is the study of bat parasites, which has a long history in Europe, for instance in French-speaking areas (France, Belgium). Bats are infected with a large diversity of parasites. Around the year 1999, c. 756 taxa were known to be associated with bats worldwide [167]. In Lanza's book, a wide range of parasitic organisms were presented, belonging to 13 groups: Myconta (two taxa), Acanthocephala (three taxa), Mallophaga (one taxon, accidental exposure), Anoplura (two taxa), Heteroptera (11 taxa), Neobacteria (c. 31 taxa), Protozoa (25 taxa), Cestoda (55 taxa), Digenea (105 taxa), Nematoda (62 taxa), Acari (324 taxa), Diptera (65 taxa) and Siphonaptera (64 taxa). This includes at least ten phyla: Acanthocephala (Spiny-headed worms), Apicomplexa, Arthropoda, Ascomycota (Ascomycete fungi), Euglenozoa, Firmicutes, Nematoda, Platyhelminthes, Protobacteria and Spirochaetes. Similar findings were noted by Stiles \& Nolan [235] in their "key catalogue" of bat parasites. In addition to the high diversity of bat parasites, these findings point out the predominant share, in the published records, of metazoan parasites. They also point out the issue of diseases in bats and the issue of bat parasites as disease vectors for their hosts. Indeed, we know that bats are hosts to a large range of infections (transmission linked with their ecology) and they seemingly are able to control these infections so that they are mostly asymptomatic. Some bat parasites (e.g. bat flies) are known to be disease vectors for their hosts [83, 129, 181, 184, 192, 261].

Among the earliest works on bat parasites in France is Étienne-Louis Geoffroy's Histoire abrégée des insectes [123], published in 1762 (Fig. 1). This book marks the starting point for research on bat parasites in France. The present paper reviews metazoan parasites reported on bats in France between 1762 and 2018, with the exception of acanthocephalans. According to the Host-Parasite Database of the Natural History Museum, London [125], no bat parasites belonging to the Acanthocephala phylum are currently known in France. In addition, hyperparasites are excluded from this paper. Nevertheless, it should be noted that bat parasites have their own parasites, such as Laboulbeniales fungi associated with bat flies or viruses of haemosporidian parasites. Some of these hyperparasites have reports from France, specifically in the department of Gard (Arthrorhynchus eucampsipodae Thaxt., 1901 and A. nycteribiae (Peyr.) Thaxt., 1931) [55, 129, 238]. The purpose of the present paper is twofold: the primary aim is to summarize the large body of published field data; and secondly to inform the reader about the geographical origin of the data and to contribute to a general overview and checklist of bat-parasite associations in France.

\section{Methods}

Initially, I used the works of nine authors: Anciaux de Faveaux [5-7], Beaucournu [28, 29], Beaucournu and Launay
[37], Hůrka [149], Lanza [167], Maa [185] and Szentiványi et al. [237]. The list of all the sources used in these papers offers an essential bibliographical guide. The online catalog of the Library of the Muséum National d'Histoire Naturelle (MNHN) was also used. I checked all available publications on each of the searched terms including a combination of France or the name of administrative departments $(n=111)$ or the names of former administrative regions of France $(n=22)$ with one of the generic names of the bat parasites, as mentioned in I parassiti dei pipistrelli (Mammalia, Chiroptera) della fauna italiana [167], Parasite diversity of European Myotis species with special emphasis on Myotis myotis (Microchiroptera, Vespertilionidae) from a typical nursery roost [121], Les puces de France et du basin méditerranéen occidental [37], and Checklist of host associations of European bat flies (Diptera: Nycteribiidae, Streblidae) [237]. For the study area, see Figure 5. I searched Google Scholar, ISI Web of Science, Hyper-Article en Ligne (HAL), Biodiversity Heritage Library (BHL), Gallica, and Archives. The collated sources $(n=237)$ were then analysed. I then proceeded to index them in terms of their chronology, taxonomy, and geography. The validity of all the taxa found was checked using the comprehensive synonymies provided by the Host-Parasite Database of the Natural History Museum, London [125] and the Inventaire National du Patrimoine Naturel of the Muséum National d'Histoire Naturelle (MNHN) [153]. The taxonomic works of Fain [106-108], Lanza [167], Neumann [197], Da Fonseca [72], Radovsky [212], Roy and Chauve [221, 222], Rudnick [223], Stiles and Nolan [235] and Theodor \& Moscona [241] were also used. The bat classification and taxonomy, in this paper, is based on Dietz et al. (2009) and Arthur and Lemaire (2015). Authorities for the host taxa and parasites species are given in Tables 1 and 2. The map in Figure 5 was created using Carto-SI (https://www.carto-si.com/).

\section{Results and discussion}

Based on published data, eight groups of bat parasites reported from France have been identified (Fig. 2). The majority of the analysed papers (94\%) were published between 1762 and 1999 (Fig. 1). All host-parasite associations are listed in Tables 1 and 2. What follows is an overview of all bat parasites, arranged by higher taxonomic group.

\section{Phylum Arthropoda Latreille, 1829}

\subsection{Subphylum Chelicerata Heymons, 1901}

\subsubsection{Subclass Acari Leach, 1817}

Most of the studied papers $(n=112)$ deal with 75 species (53 generally recognised species and 22 invalid species) of Acari of four groups: Ixodida, Mesostigmata, Trombidiformes (suborder Prostigmata), and Sarcoptiformes (Fig. 3). A total of 53 recognised species (including two subspecies) of mites and ticks were reported to be parasites of bats in France prior to 2018. Of these, two species have only been collected from the border between Switzerland and the French department of Haute-Savoie (Col de Bretolet): Spinturnix helvetiae and S. acuminatus. 


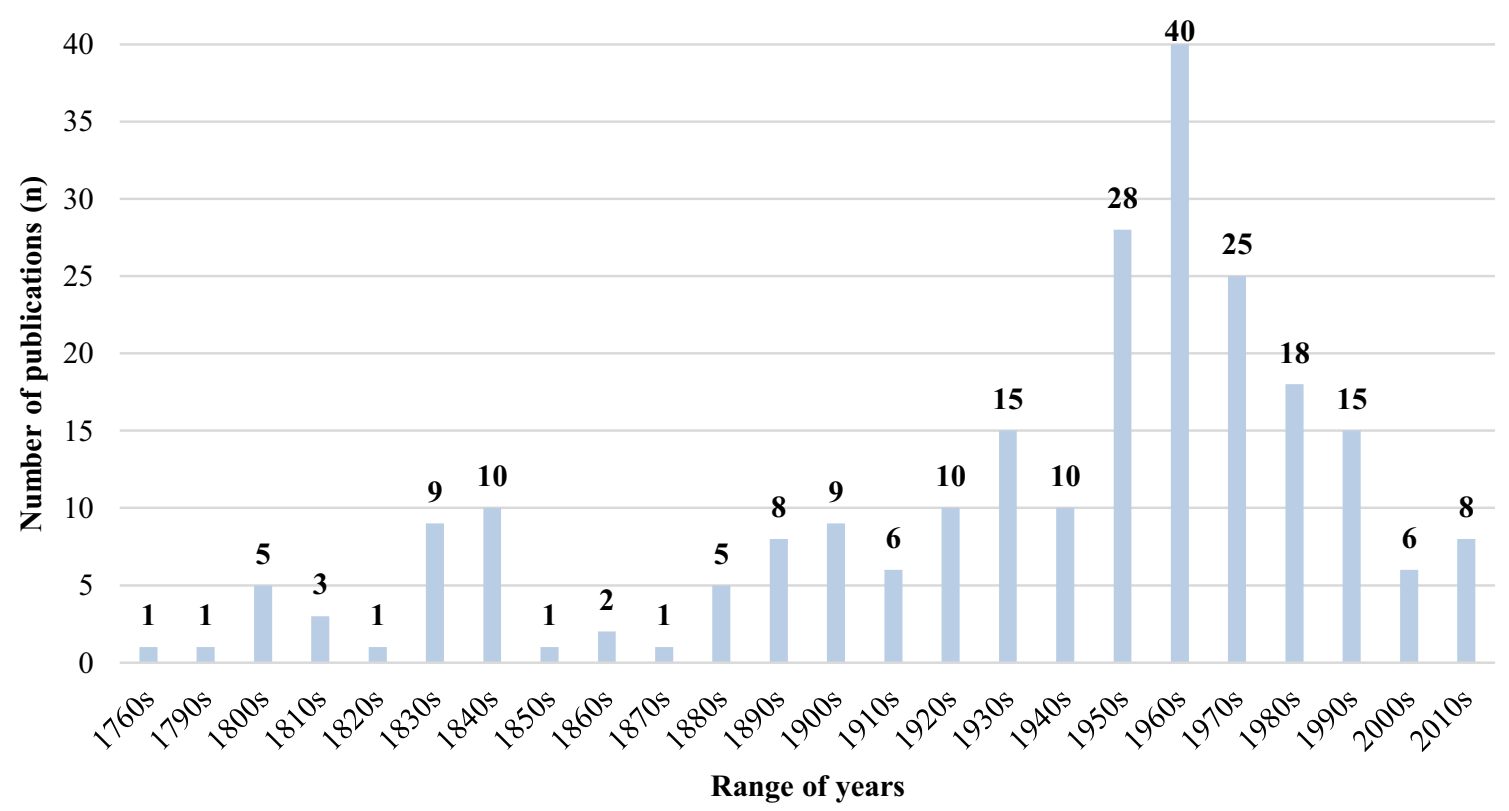

Figure 1. Number of studies $(n=237)$ that include bat parasites observed in France since 1760, by decade.

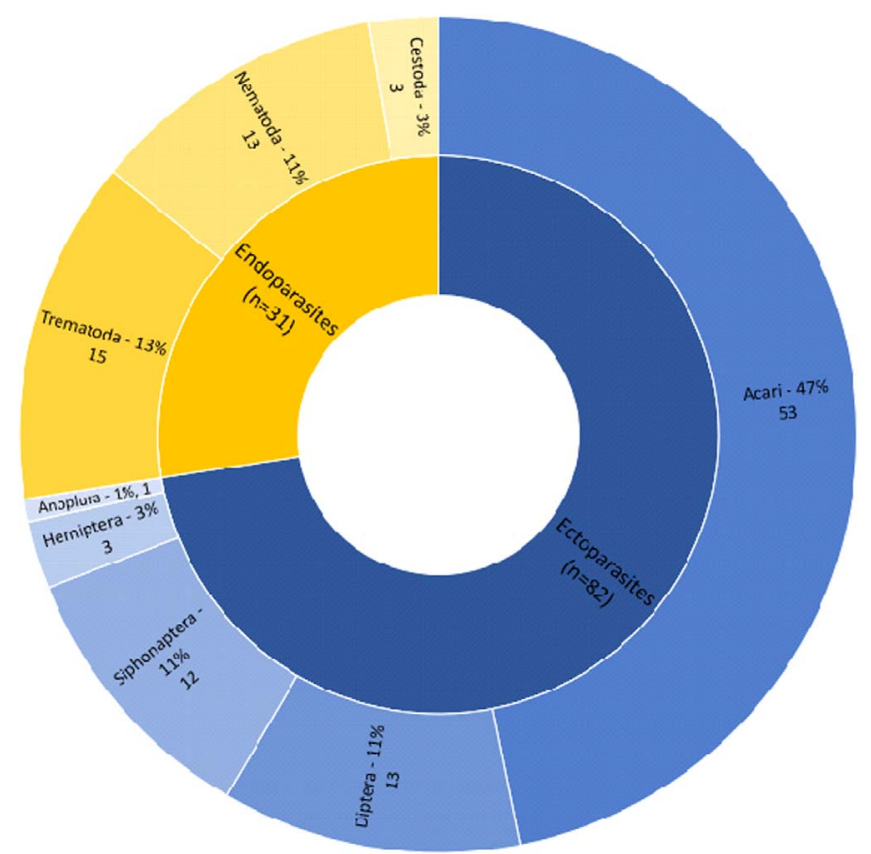

Figure 2. Overview of the 113 generally recognised parasite taxa that are mentioned in the analysed papers $(n=237)$ per host taxonomic group. Invalid species ( $n=22$ Acari and 3 Diptera) recorded in the literature, records reported from France without identification to species level ( $n=6$ Acari; 1 Cestoda; 2 Diptera; 1 Hemiptera; 2 Nematoda and 2 Trematoda) and species only noted as absent $(n=3$ Acari and 1 Diptera) are not included here.

These recognised species found in the literature $(n=53)$ reported from France belong to 23 genera: Acanthophthirius, Alabidocarpus, Argas, Calcarmyobia, Eyndhovenia, Hirstionyssus, Ichoronyssus, Ixodes, Labidocarpus, Leptotrombidium, Macronyssus, Neomyobia, Neotrombicula, Notoedres, Nycteridocoptes, Oudemansidium, Paraperiglischrus, Pteracarus, Psorergates, Riedlinia, Sasatrombicula, Steatonyssus and Spinturnix. Among this group, the most diverse genera reported in France are Spinturnix and Macronyssus (Fig. 3): these genera account for $30 \%$ of all documented Acari infections involving valid species.

Six more records reported from France without identification to species level were found. These comprise Dermanyssus sp. [86], Ichoronyssus sp. [25], Ixodes sp. [178], Neomyobia sp. [126], Steatonyssus sp. [152, 212] and Spinturnix sp. [133]. Three other species are noted as absent from bats in western 
Table 1. List of bat species and their associated metazoan parasites in France (including Corsica), based on the published literature. Authors are listed in the bibliography. See also the work titled Les parasites métazoaires des Chiroptères de France (Acari, Anoplura, Cestoda, Diptera, Hemiptera, Nematoda, Siphonaptera, Trematoda) : contribution à un état des lieux bibliographique (1762-2018) et à l'établissement d'une liste nationale (2019). Invalid species are listed in brackets. Records marked with an exclamation mark (!) are invalid. Records marked with a question mark (?) are dubious. They may require further clarification.

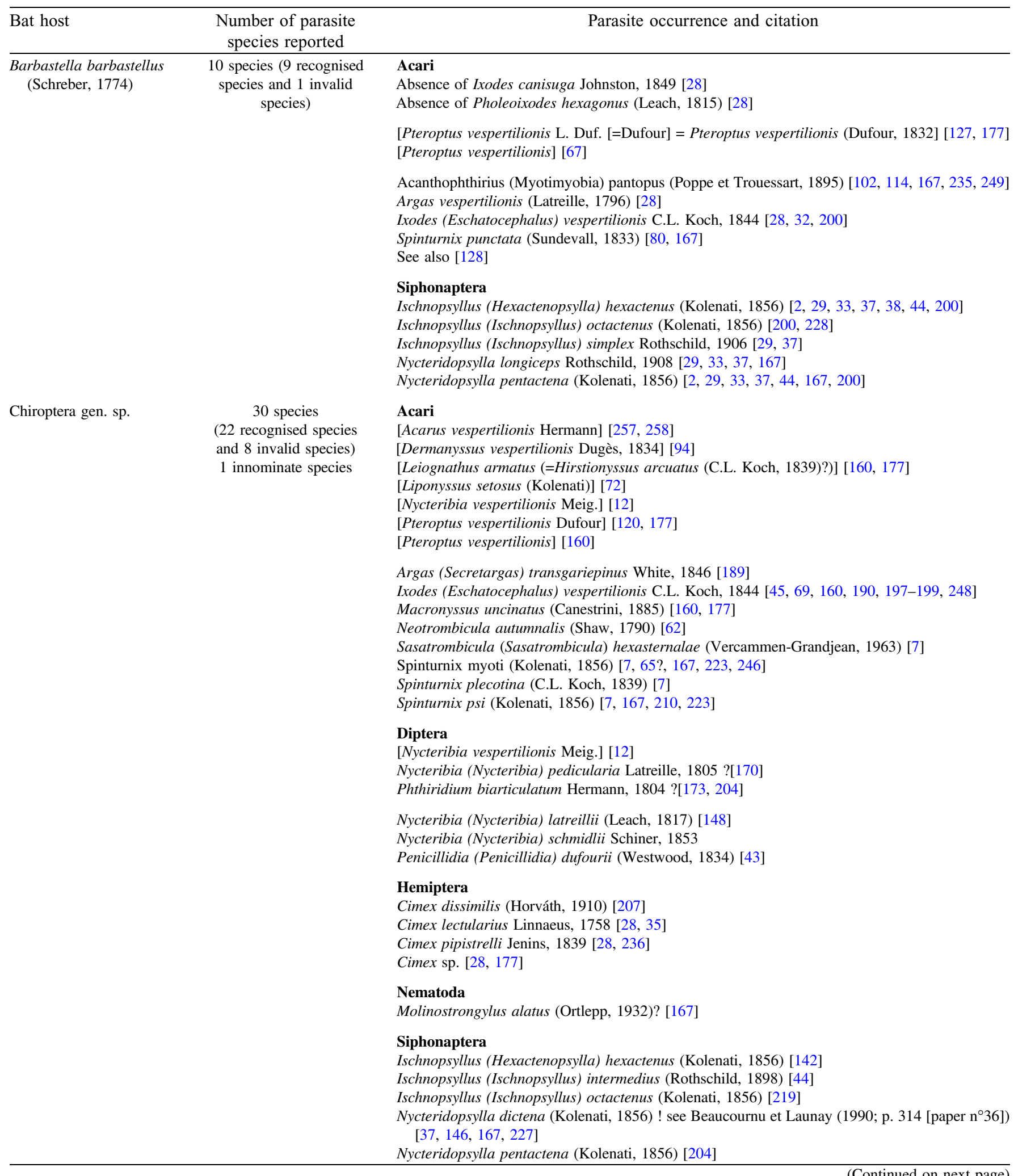


Table 1. (Continued)

\begin{tabular}{|c|c|c|}
\hline Bat host & $\begin{array}{l}\text { Number of parasite } \\
\text { species reported }\end{array}$ & Parasite occurrence and citation \\
\hline $\begin{array}{r}\text { Eptesicus serotinus } \\
\text { (Schreber, 1774) }\end{array}$ & $\begin{array}{c}17 \text { species } \\
\text { (16 recognised species } \\
\text { and } 1 \text { invalid species) } \\
1 \text { innominate species }\end{array}$ & $\begin{array}{l}\text { Acari } \\
\text { Absence of Ixodes canisuga Johnston, } 1849[28] \\
\text { Absence of Pholeoixodes hexagonus (Leach, 1815) [28] } \\
\text { [Pteroptus vespertilionis (Dufour, 1832)] [190] } \\
\text { Argas (Carios) vespertilionis (Latreille, 1802) [31, 45, 86] } \\
\text { Argas (Secretargas) transgariepinus White, 1846 [45, 189] } \\
\text { Argas vespertilionis (Latreille, 1796) [28, 38] } \\
\text { Ixodes (Eschatocephalus) vespertilionis C.L. Koch, 1844 [28, 32, 166] } \\
\text { Notoedres (Notoedres) chiropteralis (Trouessart, 1896) [48, 106, 167, 250] } \\
\text { Spinturnix psi (Kolenati, 1856) [48] } \\
\text { Cestoda } \\
\text { Vampirolepis acuta (Rudolphi, 1819) [158, 159, 177, 196, 255] } \\
\text { Vampirolepis balsaci (Joyeux \& Baer, 1934) [158, 159, 167, 177, 180, 196, 255] } \\
\text { Vampirolepis sp./Milina sp. [86] } \\
\text { Diptera } \\
\text { Basilia (Basilia) mediterranea Hůrka, 1970 [43, 167, 237] } \\
\text { Basilia (Basilia) nattereri (Kolenati, 1857) [241] } \\
\text { Nycteribia (Nycteribia) kolenatii Theodor \& Moscona, 1954 [147, 241] } \\
\text { Siphonaptera } \\
\text { Ischnopsyllus (Ischnopsyllus) intermedius (Rothschild, 1898) [29, 33, 37, 38, 142, 167, 227] } \\
\text { Nycteridopsylla pentactena (Kolenati, 1856) [29, 33, 37, 164, 167] } \\
\text { Trematoda } \\
\text { Lecithodendrium linstowi Dollfus, 1931 [68, 88, 167] } \\
\text { Plagiorchis vespertilionis (O.F. Müller, 1784) [68, 167] } \\
\text { Prosthodendrium (Prosthodendrium) longiforme (Bhalerao, 1926) [88, 167] }\end{array}$ \\
\hline Eptesicus sp. & 1 recognised species & $\begin{array}{l}\text { Acari } \\
\text { Neotrombicula racovitzai Feider, } 1970[6,118,167]\end{array}$ \\
\hline $\begin{array}{l}\text { Hypsugo savii (Bonaparte, } \\
\text { 1837) }\end{array}$ & 4 recognised species & $\begin{array}{l}\text { Acari } \\
\text { Argas (Carios) vespertilionis (Latreille, 1802) [45] } \\
\text { Argas (Secretargas) transgariepinus White, } 1846[45,189] \\
\text { Spinturnix nobleti Deunff, Volleth, Keller et Aellen, } 1990 \text { [81, 167] } \\
\text { Diptera } \\
\text { Basilia (Basilia) mediterranea Hůrka, } 1970[41,167,237]\end{array}$ \\
\hline $\begin{array}{l}\text { Miniopterus schreibersii } \\
\text { (Natterer in Kuhl, } \\
\text { 1817) }\end{array}$ & $\begin{array}{c}38 \text { species } \\
\text { (33 recognised species } \\
\text { and } 5 \text { invalid species) }\end{array}$ & $\begin{array}{l}\text { Acari } \\
\text { [Pteroptus vespertilionis (Dufour, 1832)] [203] } \\
\text { [Spinturnix vespertilionis] [24] } \\
\text { [Spinturnix vespertilionis (C.L. Koch)] [70] } \\
\text { Argas vespertilionis (Latreille, 1796) ? [85] } \\
\text { Calcarmyobia rhinolophia (Radford, 1940)? There is only one case. According to Lanza (1999), } \\
\quad \text { this field data published by Beron (1971) is doubtful: it could be Calcarmyobia dusbabeki } \\
\quad \text { Uchikawa, } 1985 \text { [50, 51, 167] } \\
\text { Eyndhovenia euryalis (G. Canestrini, 1885) [76] } \\
\text { Ichoronyssus diversipilis Vitzthum, 1920 [24] } \\
\text { Ichoronyssus spinosus (Oudemans) 1902) [24, 70, 72] } \\
\text { Ixodes (Eschatocephalus) simplex simplex Neumann, } 1906 \text { [15, 31, 32, 45, 51, 143, 166, 167, } \\
\quad \text { 176] see Beaucournu (1966; p. 498) paper n⿳30 } 30 \\
\text { Ixodes (Eschatocephalus) vespertilionis C.L. Koch, 1844 [6, 28, 176]! } \\
\quad \text { see Beaucournu (1966; p. 498) paper n }{ }^{\circ} 30 \text { [200, 242] } \\
\text { Macronyssus granulosus (Kolenati, 1856) [50, 167] } \\
\text { Spinturnix myoti (Kolenati, 1856) [76] } \\
\text { Spinturnix psi (Kolenati, 1856) [50, 63, 76, 139] } \\
\text { Cestoda } \\
\text { Milina grisea van Beneden, 1873 [68, 86, 167, 200?] } \\
\text { Diptera } \\
\text { [Nycteribia vespertilionis Meig.] [203] } \\
\text { Nycteribia (Nycteribia) pedicularia Latreille, 1805 ! [85] } \\
\text { Phthiridium biarticulatum Hermann, 1804 ? [85, 126, 237] }\end{array}$ \\
\hline
\end{tabular}


Table 1. (Continued)

\begin{tabular}{|c|c|c|}
\hline Bat host & $\begin{array}{c}\text { Number of parasite } \\
\text { species reported }\end{array}$ & Parasite occurrence and citation \\
\hline & & $\begin{array}{l}\text { Nycteribia (Nycteribia) kolenatii Theodor \& Moscona, } 1954 \text { [200] } \\
\text { Nycteribia (Nycteribia) latreillii (Leach, 1817) [1, 167] } \\
\text { Nycteribia (Nycteribia) schmidlii Schiner, 1853 [1, 24, 28, 30, 43, 115, 149, 154, 167, 242]; see } \\
\quad \text { also [117, 150] } \\
\text { Nycteribia (Achrocholidia) vexata Westwood, } 1835[1,43,85,167] \\
\text { Penicillidia (Penicillidia) dufourii (Westwood, 1834) [1, 24, 30, 237] } \\
\text { Penicillidia (Neopenicillidia) conspicua Speiser, 1901 ([1, 28, 30, 115, 154, 167, 242], see also } \\
\quad[117,150,237]) \\
\text { Nematoda } \\
\text { Litomosa ottavianii Lagrange \& Bettini, 1948 [19, 167] } \\
\text { Molinostrongylus panousei Dollfus, 1954 [99, 167] } \\
\text { Molinostrongylus tipula (Beneden, 1873) [200] } \\
\text { Riouxgolvania rhinolophi (Bain \& Chabaud, 1968) [21, 167] } \\
\text { Strongylacantha glycirrhyza van Beneden, 1873 [99, 167, 200] } \\
\text { Trichosomum speciosum van Beneden, 1873 [200] } \\
\text { See also [174] } \\
\text { Siphonaptera } \\
\text { Nycteridopsylla pentactena (Kolenati, 1856) [37, 164, 167] } \\
\text { Trematoda } \\
\text { [Paralecithodendrium chilostomum (Mehlis) (=Prosthodendrium (Prosthodendrium) chilostomum } \\
\quad \text { (Mehlis, 1831) ?)] [200] } \\
\text { Lecithodendrium granulosum Looss, 1907 ? [200] } \\
\text { Lecithodendrium linstowi Dollfus, 1931 [68, 167] } \\
\text { Mesotretes peregrinus (Braun, 1900) [68, 167, 188] } \\
\text { Parabascus lepidotus Looss, 1907 [68, 167] } \\
\text { Plagiorchis vespertilionis (O.F. Müller, 1784) [68, 167, 200, 235] } \\
\text { Prosthodendrium (Prosthodendrium) chilostomum (Mehlis, 1831) [68, 167, 200, 235] } \\
\text { Prosthodendrium parvouterus (Bhalerao, 1926) [68, 167] } \\
\text { See also [174] }\end{array}$ \\
\hline $\begin{array}{l}\text { Myotis blythii (Tomes, } \\
\text { 1857) and M. blythii } \\
\text { oxygnathus Monticelli, } \\
1885\end{array}$ & $\begin{array}{l}9 \text { recognised species } \\
1 \text { innominate species }\end{array}$ & $\begin{array}{l}\text { Acari } \\
\text { Absence of Ixodes (Eschatocephalus) simplex simplex Neumann, } 1906 \text { [32] } \\
\text { Nycteridocoptes poppei Oudemans, } 1898[113] \\
\text { Spinturnix myoti (Kolenati, 1856) [14, 76] } \\
\text { Diptera } \\
\text { Nycteribia (Achrocholidia) vexata Westwood, } 1835[30] \\
\text { Nycteribia (Nycteribia) latreillii (Leach, 1817) [30, 167] } \\
\text { Nycteribia (Nycteribia) pedicularia Latreille, } 1805[115,154,167] \\
\text { Nycteribia (Nycteribia) schmidlii Schiner, 1853 [30] } \\
\text { Penicillidia (Penicillidia) dufourii (Westwood, 1834) [14, 43, 115, 154, 237] } \\
\text { Nematoda } \\
\text { Riouxgolvania nyctali (Bain \& Chabaud, 1979) [21, 167] } \\
\text { Riouxgolvania sp. [86] } \\
\text { Siphonaptera } \\
\text { Ischnopsyllus (Hexactenopsylla) hexactenus (Kolenati, 1856) ? [14] }\end{array}$ \\
\hline $\begin{array}{l}\text { Myotis capaccinii } \\
\quad \text { (Bonaparte, } 1837)\end{array}$ & 9 recognised species & $\begin{array}{l}\text { Acari } \\
\quad \text { Spinturnix myoti (Kolenati, 1856) [73] } \\
\text { Spinturnix psi (Kolenati, 1856) }[63,76,121,139]\end{array}$ \\
\hline
\end{tabular}


Table 1. (Continued)

\begin{tabular}{|c|c|c|}
\hline Bat host & $\begin{array}{l}\text { Number of parasite } \\
\text { species reported }\end{array}$ & Parasite occurrence and citation \\
\hline & & $\begin{array}{l}\text { Diptera } \\
\text { Nycteribia (Nycteribia) pedicularia Latreille, } 1805[1,30,85] \\
\text { Penicillidia (Penicillidia) dufourii (Westwood, 1834) [1,237] } \\
\text { Siphonaptera } \\
\text { Nycteridopsylla longiceps } \text { Rothschild, } 1908[37,164,167] \\
\text { Nycteridopsylla pentactena (Kolenati, 1856) [37, 164, 167] } \\
\text { Trematoda } \\
\text { Lecithodendrium granulosum Looss, } 1907[68] \\
\text { Lecithodendrium linstowi } \text { Dollfus, } 1931[68,167] \\
\text { Plagiorchis vespertilionis } \text { (O.F. Müller, 1784) [68, 167] }\end{array}$ \\
\hline $\begin{array}{l}\text { Myotis dasycneme (Boie, } \\
\text { 1825) }\end{array}$ & 1 recognised species & $\begin{array}{l}\text { Acari } \\
\text { Argas (Carios) vespertilionis (Latreille, 1802) [6, 31, 85] }\end{array}$ \\
\hline $\begin{array}{l}\text { Myotis daubentonii } \\
\quad \text { (Leisler in Kuhl, 1817) } \\
\text { and Myotis daubentonii } \\
\text { nathalinae }\end{array}$ & $\begin{array}{c}19 \text { species (17 recognised } \\
\text { species and } 2 \text { invalid } \\
\text { species) } \\
1 \text { innominate species }\end{array}$ & $\begin{array}{l}\text { Acari } \\
\text { Absence of Ixodes canisuga Johnston, } 1849 \text { [28] } \\
\text { Absence of Pholeoixodes hexagonus (Leach, 1815) [28] } \\
\text { [Pteroptus trouessarti Mégnin (=Haemomyson trouessarti (Mégnin)?)] [248] } \\
\text { Ixodes (Eschatocephalus) vespertilionis C.L. Koch, 1844 [28, 32] } \\
\text { Leptotrombidium russicum (Oudemans, 1902) [122, 167, 177, 256] see also [10] } \\
\text { Neotrombicula autumnalis (Shaw, 1790) [8-10,122, 167, 177, 256] } \\
\text { Nycteridocoptes poppei Oudemans, 1898 [113] } \\
\text { Oudemansidium musca (Oudemans, 1906) [122, 167, 177] } \\
\text { Spinturnix andegavinus (Deunff, 1977) [76] } \\
\text { Diptera } \\
\text { [Nycteribia eparticulata] [248] } \\
\text { Nycteribia (Nycteribia) pedicularia Latreille, 1805 ! [85] } \\
\text { Basilia (Basilia) nattereri (Kolenati, 1857) [187] } \\
\text { Nycteribia (Nycteribia) kolenatii Theodor \& Moscona, 1954 [1, 28, 38, 43, 167, 241] } \\
\text { Nycteribia (Nycteribia) schmidlii Schiner, 1853 [147] } \\
\text { Nycteribia sp. [247] } \\
\text { Siphonaptera } \\
\text { Ischnopsyllus (Ischnopsyllus) simplex Rothschild, 1906 [44] } \\
\text { Ischnopsyllus (Ischnopsyllus) variabilis (Wagner, 1898) [44] } \\
\text { Nycteridopsylla longiceps Rothschild, 1908 [29, 37, 167] } \\
\text { Trematoda } \\
\text { Allassogonoporus amphoraeformis (Mödlinger, 1930) [89, 167] } \\
\text { Parabascus duboisi (Hurkova, 1961) [89, 167] } \\
\text { Plagiorchis vespertilionis (O.F. Müller, 1784) [89, 167] } \\
\text { Prosthodendrium (Prosthodendrium) hurkovaae Dubois, 1960 [89] }\end{array}$ \\
\hline $\begin{array}{r}\text { Myotis emarginatus } \\
\text { (Geoffroy, 1806) }\end{array}$ & $\begin{array}{l}19 \text { recognised species } \\
2 \text { innominate species }\end{array}$ & $\begin{array}{l}\text { Acari } \\
\text { Absence of Ixodes canisuga Johnston, } 1849[28] \\
\text { Absence of Pholeoixodes hexagonus (Leach, 1815) [28] } \\
\text { Argas (Secretargas) transgariepinus White, } 1846[45,189] \\
\text { Argas vespertilionis (Latreille, 1796) [28] } \\
\text { Dermanyssus } \text { sp. [86] } \\
\text { Eyndhovenia euryalis (G. Canestrini, 1885) [63, 121] } \\
\text { Ixodes (Eschatocephalus) vespertilionis C.L. Koch, } 1844 \text { [28, 32, 38, 136, 137, 177] } \\
\text { Spinturnix emarginatus (Kolenati, 1856) [7, 76, 78] } \\
\text { Cestoda } \\
\text { Milina grisea van Beneden, } 1873 \text { [68, 86, 167] } \\
\text { Diptera } \\
\text { Nycteribia (Achrocholidia) vexata Westwood, } 1835 ![23] \\
\text { Nycteribia (Nycteribia) pedicularia Latreille, 1805 ? [23] } \\
\text { Basilia (Basilia) italica Theodor, } 1954 \text { [4, 34] } \\
\text { Basilia (Basilia) nana Theodor \& Moscona, 1954 [43, 237] } \\
\text { Nycteribia (Nycteribia) latreillii (Leach, 1817) [22] }\end{array}$ \\
\hline
\end{tabular}


Table 1. (Continued)

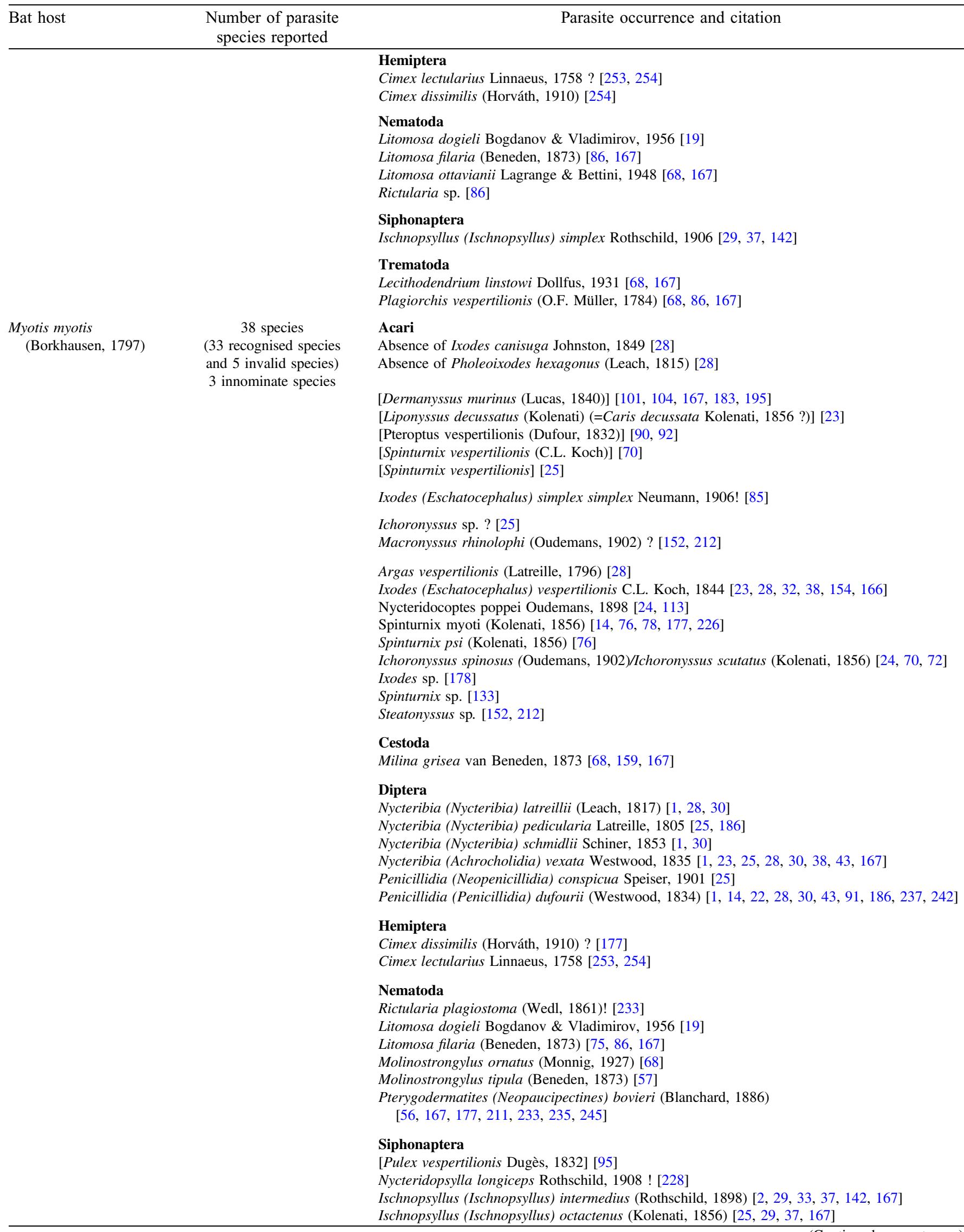


Table 1. (Continued)

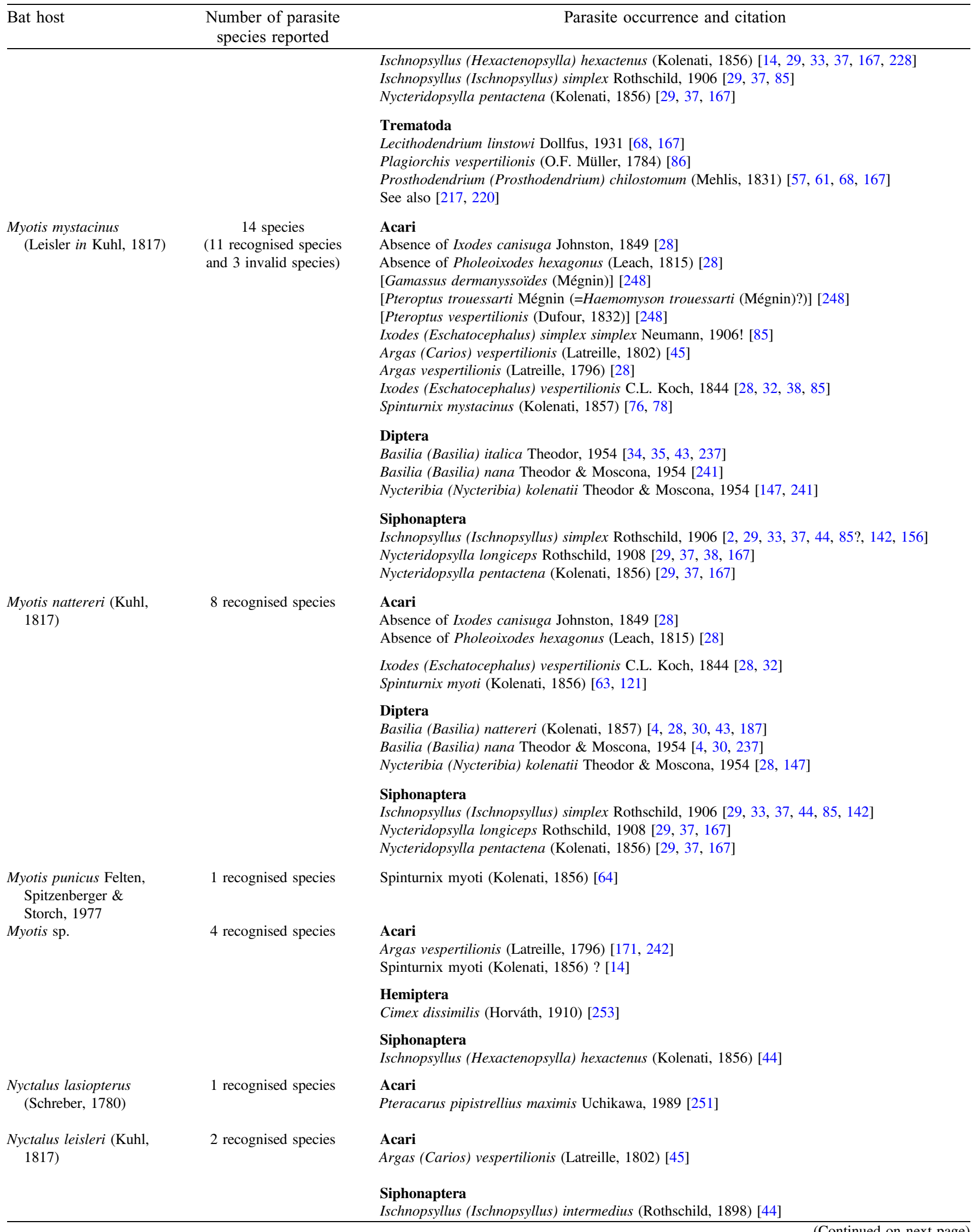


Table 1. (Continued)

\begin{tabular}{|c|c|c|}
\hline Bat host & $\begin{array}{c}\text { Number of parasite } \\
\text { species reported }\end{array}$ & Parasite occurrence and citation \\
\hline $\begin{array}{l}\text { Nyctalus noctula } \\
\quad \text { (Schreber, 1774) }\end{array}$ & $\begin{array}{l}14 \text { species ( } 8 \text { recognised } \\
\text { species and } 6 \text { invalid } \\
\text { species) }\end{array}$ & $\begin{array}{l}\text { Acari } \\
\text { [Acarus vespertilionis Hermann, } 1804 \text { - la Mite de la chauve-souris] [135] } \\
\text { [Caris vespertilionis = Argas vespertilionis (Latreille, 1796) ?] ? [168,171, 201] } \\
\text { [Dermanyssus coriaceus Gervais (=Hirstionyssus arcuatus (Koch, 1839)?)] [11,13, 103, 212, } \\
\quad 221,222,258,259] \\
\text { [Haemomyson trouessarti (Mégnin) (=Leiognathus arcuatus=Hirstionyssus arcuatus (Koch, } \\
\quad \text { 1839)? See [244]] } \\
\text { [Pteroptus vespertilionis (Dufour, 1832)] [131, 177] } \\
\text { [Pteroptus vespertilionis] [131] } \\
\text { Hirstionyssus arcuatus (Koch, 1839) [11,12,103, 124, 127, 167, 177, 212, 221, 258, 259] } \\
\text { Spinturnix helvetiae (Deunff, Aellen \& Keller, 1986) [recorded at Col de Bretolet, at the border } \\
\quad \text { between France and Switzerland] [79] } \\
\text { Spinturnix acuminatus (C.L. Koch, 1836) [recorded at Col de Bretolet, at the border between } \\
\quad \text { France and Switzerland] [79] } \\
\text { Diptera } \\
\text { Nycteribia (Nycteribia) pedicularia Latreille, 1805![85] } \\
\text { Siphonaptera } \\
\text { Ischnopsyllus (Ischnopsyllus) variabilis (Wagner, 1898) ? [37] } \\
\text { Ischnopsyllus (Ischnopsyllus) elongatus (Curtis, 1832) [37, 42, 44, 167, 204, 228!, 231!] } \\
\text { Ischnopsyllus (Ischnopsyllus) intermedius (Rothschild, 1898) [3, 44] } \\
\text { Nycteridopsylla eusarca Dampf, 1908 [37, 42] }\end{array}$ \\
\hline $\begin{array}{l}\text { Pipistrellus kuhlii (Kuhl, } \\
\text { 1817) }\end{array}$ & 9 recognised species & $\begin{array}{l}\text { Acari } \\
\text { Absence of Ixodes canisuga Johnston, } 1849 \text { [28] } \\
\text { Absence of Ixodes (Eschatocephalus) simplex simplex Neumann, 1906 [32] } \\
\text { Absence of Pholeoixodes hexagonus (Leach, 1815) [28] } \\
\text { Argas (Carios) vespertilionis (Latreille, 1802) [45] } \\
\text { Argas vespertilionis (Latreille, 1796) [28, 166] } \\
\text { Ixodes (Eschatocephalus) vespertilionis C.L. Koch, } 1844 \text { [28] } \\
\text { Siphonaptera } \\
\text { Ischnopsyllus (Hexactenopsylla) hexactenus (Kolenati, 1856) [29] } \\
\text { Ischnopsyllus (Ischnopsyllus) octactenus (Kolenati, 1856) [29, 33, 37, 167] } \\
\text { Ischnopsyllus (Ischnopsyllus) variabilis (Wagner, 1898) [37, 44, 142] } \\
\text { Nycteridopsylla longiceps Rothschild, 1908 [37, 44, 156] } \\
\text { Trematoda } \\
\text { Lecithodendrium linstowi Dollfus, 1931 [86] } \\
\text { Pycnoporus heteroporus (Dujardin, 1845) [86] }\end{array}$ \\
\hline $\begin{array}{l}\text { Pipistrellus nathusii } \\
\text { (Keyserling \& Blasius, } \\
\text { 1839) }\end{array}$ & 8 recognised species & $\begin{array}{l}\text { Acari } \\
\text { Absence of Ixodes canisuga Johnston, } 1849 \text { [28] } \\
\text { Absence of Ixodes (Eschatocephalus) simplex simplex Neumann, } 1906 \text { [32] } \\
\text { Absence of Pholeoixodes hexagonus (Leach, 1815) [28] } \\
\text { Acanthophthirius (Acanthophthirius) poppei (Trouessart, 1895) [52, 102, 114, 167, 249] } \\
\text { Argas (Carios) vespertilionis (Latreille, 1802) [45] } \\
\text { Argas vespertilionis (Latreille, 1796) [28] } \\
\text { Ixodes (Eschatocephalus) vespertilionis C.L. Koch, } 1844 \text { [28] } \\
\text { Siphonaptera } \\
\text { Ischnopsyllus (Ischnopsyllus) octactenus (Kolenati, 1856) [29, 33, 37, 167] } \\
\text { Ischnopsyllus (Ischnopsyllus) variabilis (Wagner, 1898) [2, 29, 33, 37, 44] } \\
\text { Nycteridopsylla longiceps Rothschild, 1908 [37, 44, 167] } \\
\text { Nycteridopsylla pentactena (Kolenati, 1856) [44] }\end{array}$ \\
\hline $\begin{array}{l}\text { Pipistrellus pipistrellus } \\
\text { (Schreber, 1774) }\end{array}$ & $\begin{array}{c}23 \text { species } \\
\text { (17 recognised species } \\
\text { and } 6 \text { invalid species and } \\
\text { one species complex) }\end{array}$ & $\begin{array}{l}\text { Acari } \\
\text { Absence of Ixodes canisuga Johnston, } 1849 \text { [28] } \\
\text { Absence of Ixodes (Eschatocephalus) simplex simplex Neumann, } 1906 \text { [32] } \\
\text { Absence of Pholeoixodes hexagonus (Leach, 1815) [28] } \\
\text { Absence of [Pteroptus vespertilionis] [67] } \\
\text { [Argas caris] [191] } \\
\text { [Caris vespertilionis = Argas vespertilionis (Latreille, 1796) ?] ? [258] } \\
\text { [Gamasus pteroptoides (Mégnin)] [190] } \\
\text { [Haemomyson trouessarti (Mégnin) (=Leiognathus arcuatus=Hirstionyssus arcuatus (Koch, } \\
\quad \text { 1839)? See [221]] } \\
\text { [Laelaps (Iphis) agilis Koch, Berlese = Gamasus pteroptoides (Mégnin)? = Laelaps agilis C.L. } \\
\quad \text { Koch, } 1836 \text { ?] ([244], see also [190]) }\end{array}$ \\
\hline
\end{tabular}


Table 1. (Continued)

\begin{tabular}{|c|c|c|}
\hline Bat host & $\begin{array}{l}\text { Number of parasite } \\
\text { species reported }\end{array}$ & Parasite occurrence and citation \\
\hline & & [Pteroptus vespertilionis Hermann] [215] \\
\hline & & $\begin{array}{l}\text { Argas (Carios) vespertilionis (Latreille, 1802) [31, 45, 177, 239] } \\
\text { Argas vespertilionis (Latreille, 1796) [18, 28, 38, 67, 166] } \\
\text { Ixodes (Eschatocephalus) vespertilionis C.L. Koch, 1844 [28, 86, 199] } \\
\text { See also [201] }\end{array}$ \\
\hline & & $\begin{array}{l}\text { Diptera } \\
\text { Nycteribia (Nycteribia) pedicularia Latreille, } 1805 ? \text { ? [214, 215] } \\
\text { Basilia (Basilia) mediterranea Hůrka, } 1970[40,43,167] \\
\text { Nycteribia (Nycteribia) kolenatii Theodor \& Moscona, 1954 [241] } \\
\text { Nycteribia (Nycteribia) schmidlii Schiner, } 1853 \text { [147] } \\
\text { Nycteribia sp. - Complex of species Nycteribia kolenatiillatreillii/pedicularia }[177,214,215]\end{array}$ \\
\hline & & $\begin{array}{l}\text { Siphonaptera } \\
\text { Ischnopsyllus (Ischnopsyllus) octactenus (Kolenati, 1856) [2, 29, 33, 36, 37, 44, 142, 164, 167, } \\
\quad 177,227] \\
\text { Ischnopsyllus (Ischnopsyllus) variabilis (Wagner, 1898) [33, 37, 85] } \\
\text { Nycteridopsylla ancyluris ancyluris Jordan, 1942 [33,37, 44, 142, 157, 164] } \\
\text { Nycteridopsylla longiceps Rothschild, } 1908[2,29,33,37,85,167] \\
\text { Nycteridopsylla pentactena (Kolenati, 1856) }[29,37,38,167]\end{array}$ \\
\hline & & $\begin{array}{l}\text { Trematoda } \\
\text { Lecithodendrium linstowi Dollfus, } 1931 \text { [68, 167] } \\
\text { Parabascus semisquamosus (Braun, 1900) [60, 68, 167] } \\
\text { Plagiorchis vespertilionis (O.F. Müller, 1784) [68, 86, 167] } \\
\text { Pycnoporus heteroporus (Dujardin, 1845) [57, 61, 68, 97, 163, 167, 235] }\end{array}$ \\
\hline $\begin{array}{l}\text { Pipistrellus sp. } \\
\text { Nota bene: according to } \\
\text { Lord } \text { et al., for studies } \\
\text { published before the } \\
\text { 1990's, Pipistrellus } \\
\text { pipistrellus and } \\
\text { Pipistrellus sp. } \\
\text { specimens should be } \\
\text { treated as potentially } \\
\text { also including } \\
\text { P. pygmaeus. }\end{array}$ & 3 recognised species & $\begin{array}{l}\text { Acari } \\
\text { Argas (Carios) vespertilionis (Latreille, 1802) [45] } \\
\text { Diptera } \\
\text { Basilia (Basilia) mediterranea Hůrka, } 1970 \text { [41] } \\
\text { Penicillidia (Penicillidia) dufourii (Westwood, 1834) [53] }\end{array}$ \\
\hline \multirow[t]{6}{*}{$\begin{array}{l}\text { Plecotus auritus (Linné, } \\
\text { 1758) }\end{array}$} & $\begin{array}{l}16 \text { recognised species } \\
(15 \text { recognised species } \\
\text { and } 1 \text { invalid species) } \\
1 \text { innominate species }\end{array}$ & $\begin{array}{l}\text { Acari } \\
\text { Absence of Ixodes canisuga Johnston, } 1849 \text { [28] } \\
\text { Absence of Ixodes (Eschatocephalus) simplex simplex Neumann, 1906 [32] } \\
\text { Absence of Pholeoixodes hexagonus (Leach, 1815) [28] } \\
\text { [Spinturnix vespertilionis] [70] } \\
\text { Argas (Carios) vespertilionis (Latreille, 1802) [45] } \\
\text { Argas vespertilionis (Latreille, 1796) [28] } \\
\text { Ixodes (Eschatocephalus) vespertilionis C.L. Koch, } 1844 \text { [28, 199] } \\
\text { Spinturnix plecotina (C.L. Koch, 1839) [63, 76, 167] } \\
\text { See also [201] }\end{array}$ \\
\hline & & $\begin{array}{l}\text { Cestoda } \\
\text { Vampirolepis sp./Milina sp. [86] }\end{array}$ \\
\hline & & $\begin{array}{l}\text { Diptera } \\
\text { Basilia (Basilia) nana Theodor \& Moscona, } 1954 \text { [28, 237] } \\
\text { Basilia (Basilia) nattereri (Kolenati, 1857) [28, 187] } \\
\text { Phthiridium biarticulatum Hermann, } 1804 \text { [85, 86, 237] }\end{array}$ \\
\hline & & $\begin{array}{l}\text { Nematoda } \\
\text { Litomosa filaria (Beneden, 1873) [19, 167] } \\
\text { Seuratum mucronatum (Rudolphi, 1809) [54, 86, 194] }\end{array}$ \\
\hline & & $\begin{array}{l}\text { Siphonaptera } \\
\text { Ischnopsyllus (Ischnopsyllus) simplex Rothschild, } 1906 ? \text { ? [29, 37] } \\
\text { Ischnopsyllus (Ischnopsyllus) variabilis! (Wagner, 1898) [85] } \\
\text { Ischnopsyllus (Hexactenopsylla) hexactenus (Kolenati, 1856) [2, 33, 37, 44, 142, 177, 227, 228] } \\
\text { Nycteridopsylla longiceps Rothschild, } 1908[33,37,85,167,227] \\
\text { Nycteridopsylla pentactena (Kolenati, 1856) [29, 33, 37, 167] }\end{array}$ \\
\hline & & $\begin{array}{l}\text { Trematoda } \\
\text { Plagiorchis vespertilionis (O.F. Müller, 1784) [86, 167] } \\
\text { Prosthodendrium } \text { sp. [86] }\end{array}$ \\
\hline
\end{tabular}


Table 1. (Continued)

\begin{tabular}{|c|c|c|}
\hline Bat host & $\begin{array}{c}\text { Number of parasite } \\
\text { species reported }\end{array}$ & Parasite occurrence and citation \\
\hline $\begin{array}{l}\text { Plecotus austriacus } \\
\text { Fischer, } 1829\end{array}$ & 7 recognised species & $\begin{array}{l}\text { Acari } \\
\text { Absence of Ixodes (Eschatocephalus) simplex simplex Neumann, } 1906 \text { [32] } \\
\text { Argas (Carios) vespertilionis (Latreille, 1802) [31] } \\
\text { Argas (Secretargas) transgariepinus White, 1846 [45, 189] } \\
\text { Spinturnix plecotina (C.L. Koch, 1839) [63, 76, 167] } \\
\text { See also [128] } \\
\text { Siphonaptera } \\
\text { Ischnopsyllus (Hexactenopsylla) hexactenus (Kolenati, 1856) [33, 37, 44] } \\
\text { Nycteridopsylla pentactena (Kolenati, 1856) [33, 37, 167] } \\
\text { Trematoda } \\
\text { Lecithodendrium linstowi Dollfus, 1931 [68, 167] } \\
\text { Parabascus lepidotus Looss, } 1907 \text { [68, 167] }\end{array}$ \\
\hline Plecotus sp. & 1 recognised species & $\begin{array}{l}\text { Nematoda } \\
\text { Seuratum mucronatum (Rudolphi, 1809) [97] }\end{array}$ \\
\hline $\begin{array}{l}\text { Rhinolophus euryale } \\
\quad \text { Blasius, } 1853\end{array}$ & 21 recognised species & 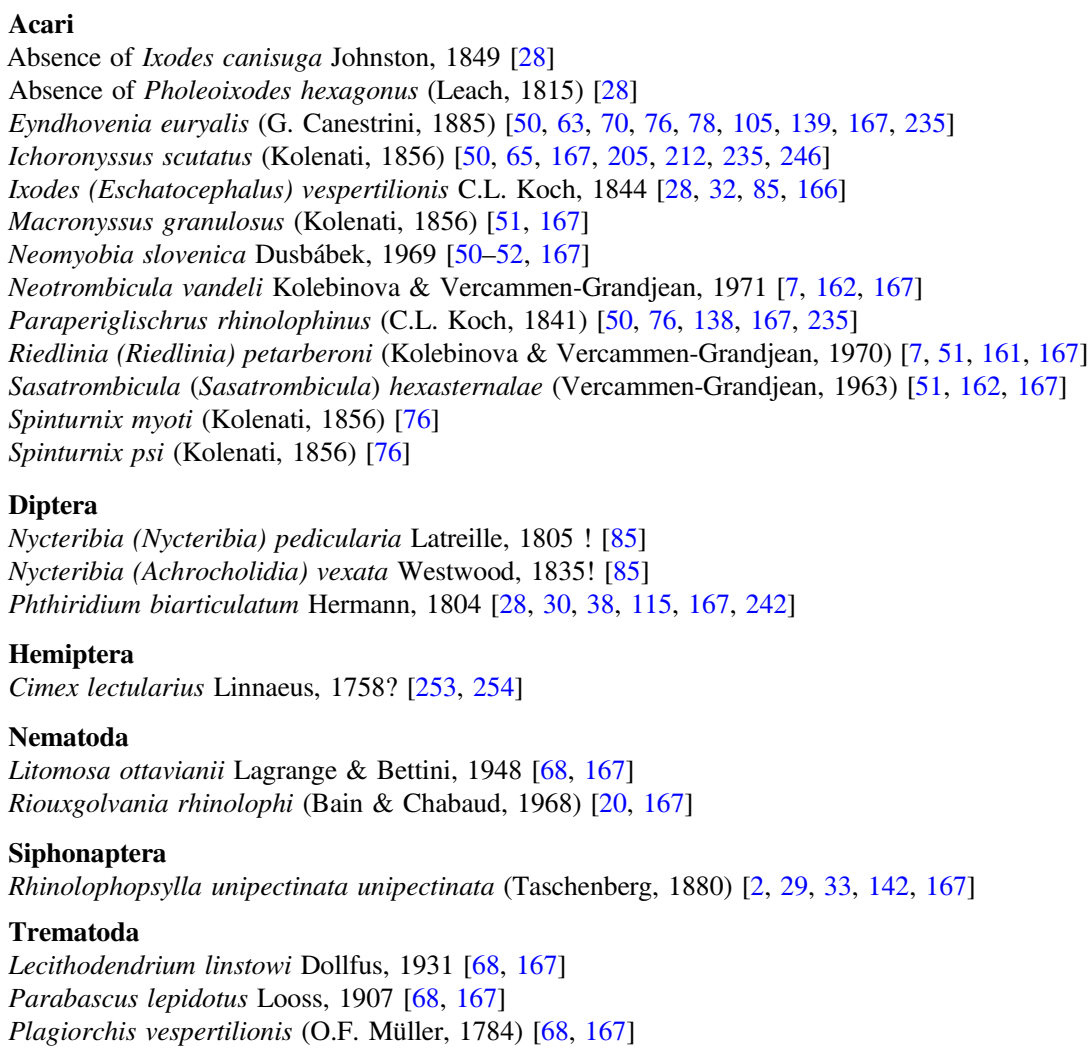 \\
\hline $\begin{array}{l}\text { Rhinolophus } \\
\quad \text { ferrumequinum } \\
\text { (Schreber, 1774) }\end{array}$ & $\begin{array}{c}50 \text { species } \\
\text { (44 recognised species } \\
\text { and } 6 \text { invalid species) } \\
2 \text { innominate species }\end{array}$ & $\begin{array}{l}\text { Acari } \\
\text { Absence of Ixodes canisuga Johnston, } 1849 \text { [28] } \\
\text { Absence of Pholeoixodes hexagonus (Leach, 1815) [28] } \\
\text { [Haemogamasus setosus (Kolenati)] [5] } \\
\text { [Liponyssus arcuatus Koch (=Hirstionyssus arcuatus (C.L. Koch, 1839) (pro parte) et } \\
\quad \text { Steatonyssus murinus (Lucas, 1840) (pro parte)] [200] } \\
\text { [Pteroptus vespertilionis Hermann] ([215], see also [177]) } \\
\text { [Pteroptus vespertilionis (Dufour, 1832)] [200] } \\
\text { Macronyssus rhinolophi (Oudemans, 1902) ? [152, 212] } \\
\text { Ixodes (Eschatocephalus) simplex simplex Neumann, 1906! [85] } \\
\text { Macronyssus ellipticus (Kolenati, 1856) ! [152, 177, 212] } \\
\text { According to Radovsky [212], this observation published by Husson \& Daum [152] is dubious. } \\
\text { Macronyssus longimanus (Kolenati)! [152, 177, 212]: according to Radovsky [212], this } \\
\quad \text { observation published by Husson \& Daum [152] is dubious. }\end{array}$ \\
\hline
\end{tabular}


Table 1. (Continued)

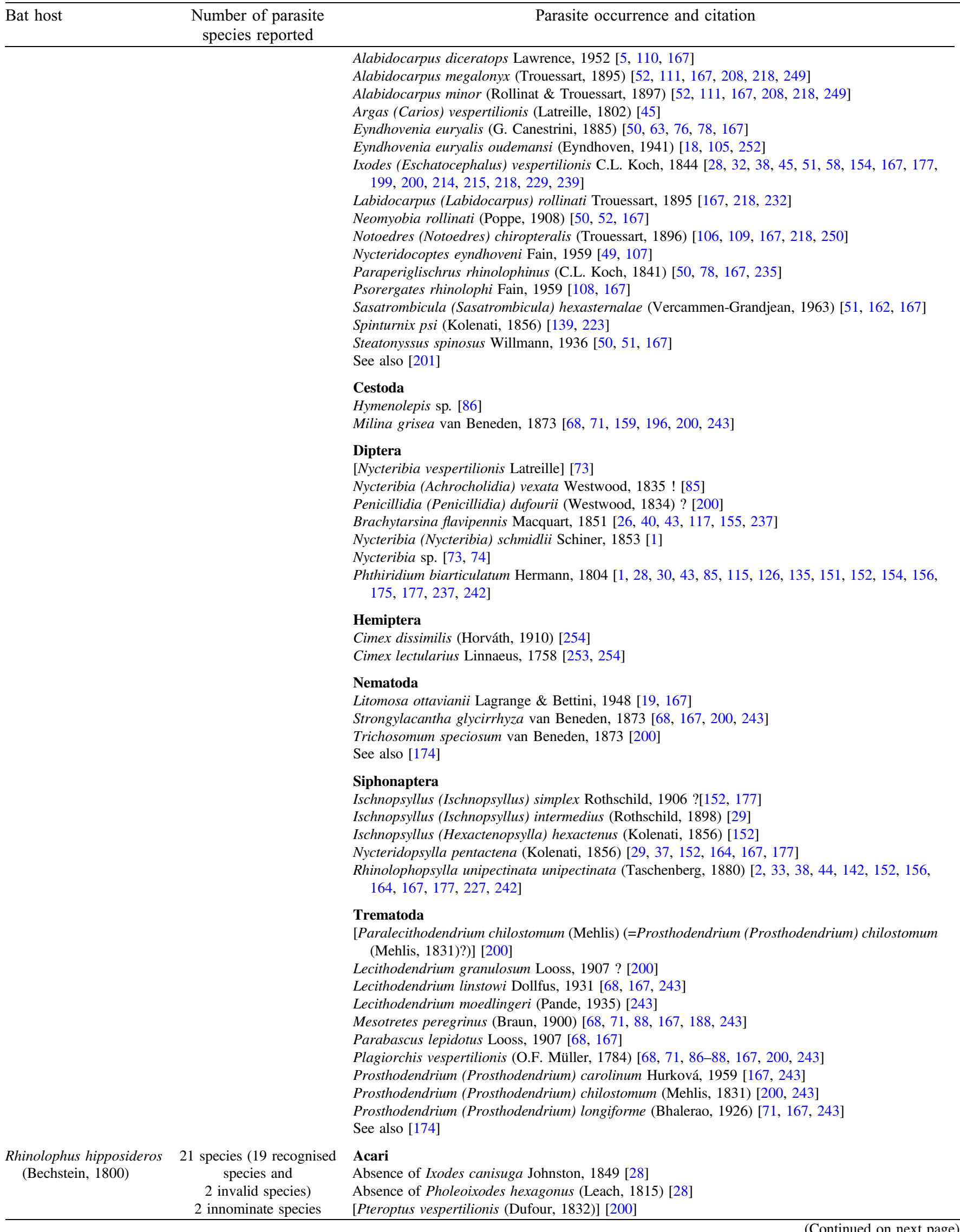


Table 1. (Continued)

\begin{tabular}{|c|c|c|}
\hline Bat host & $\begin{array}{l}\text { Number of parasite } \\
\text { species reported }\end{array}$ & Parasite occurrence and citation \\
\hline & & 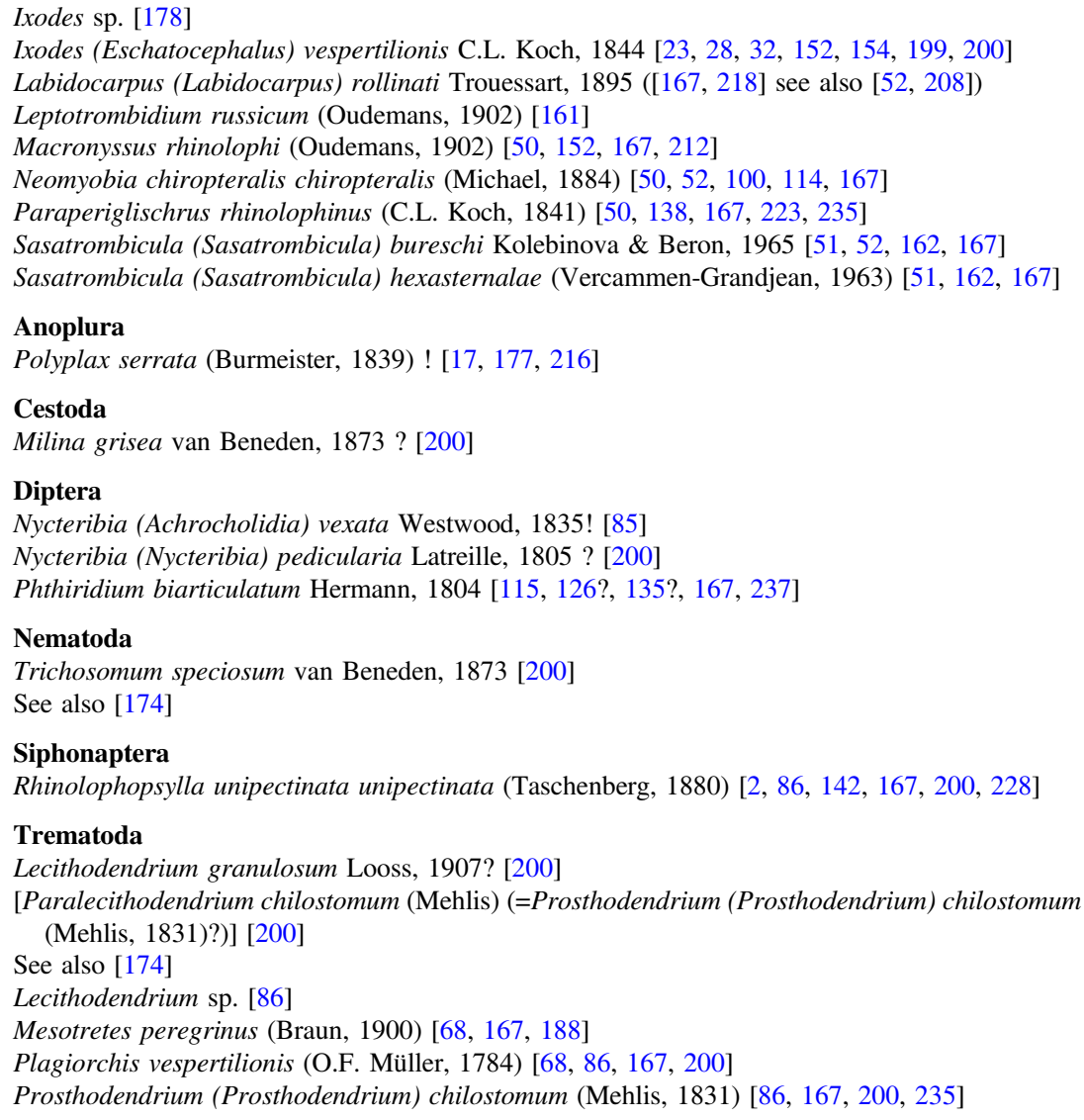 \\
\hline $\begin{array}{l}\text { Rhinolophus mehelyi } \\
\quad \text { Matschie, } 1901\end{array}$ & 1 recognised species & $\begin{array}{l}\text { Diptera } \\
\text { Nycteribia (Nycteribia) schmidlii Schiner, } 1853 \text { [147] }\end{array}$ \\
\hline Rhinolophus sp. & $\begin{array}{c}5 \text { species }(4 \text { recognised } \\
\text { species and } 1 \text { invalid } \\
\text { species) } 1 \text { innominate } \\
\text { species }\end{array}$ & $\begin{array}{l}\text { Acari } \\
\text { Eyndhovenia euryalis (G. Canestrini, 1885) [51, 76] } \\
\text { Ixodes (Eschatocephalus) vespertilionis C.L. Koch, } 1844 \text { [5, 58, 126, 197] } \\
\text { [Liponyssus setosus (Kolenati)] [72] } \\
\text { Neomyobia } \text { sp. [126] } \\
\text { Nematoda } \\
\text { Litomosa ottavianii Lagrange \& Bettini, } 1948[19,167] \\
\text { Siphonaptera } \\
\text { Rhinolophopsylla unipectinata unipectinata (Taschenberg, 1880) [44] }\end{array}$ \\
\hline $\begin{array}{l}\text { Tadarida teniotis } \\
\quad \text { (Rafinesque, 1814) }\end{array}$ & 1 recognised species & $\begin{array}{l}\text { Siphonaptera } \\
\text { Araeopsylla gestroi (Rothschild, 1906) }[3,37,44,85]\end{array}$ \\
\hline
\end{tabular}

France, namely Ixodes canisuga, I. ricinus, and Pholeoixodes hexagonus [28]. Finally, 22 invalid taxa reported from France were found in the analysed papers. Examples for this category are Dermanyssus murinus (Lucas, 1840) [101, 104, 167, 183, 195], D. vespertilionis Dugès, 1834 [94], Pteroptus vespertilionis, and Spinturnix vespertilionis (C.L. Koch)] [70].

A large host group comprising the following taxa was identified with Acari infections: Barbastella barbastellus, Eptesicus serotinus, Eptesicus sp., Hypsugo savii, Miniopterus schreibersii, Myotis bechsteinii, M. blythii, M. blythii oxygnathus, M. capaccinii, $M$. dasycneme, $M$. daubentonii, M. emarginatus, M. myotis, M. mystacinus, M. nattereri, M. punicus, Myotis sp.,
Nyctalus lasiopterus, N. leisleri, N. noctula, Pipistrellus kuhlii, P. nathusii, P. pipistrellus, Pipistrellus sp., Plecotus auritus, $P$. austriacus, Rhinolophus euryale, $R$. ferrumequinum, $R$. hipposideros and Rhinolophus sp. (see Tables 1 and 2). The oldest works dealing with Acari parasitising bats in France are Geoffroy's Histoire abrégée des insectes [123] and Latreille's Précis des caractères génériques des insectes, disposés dans un ordre naturel [168], dated 1762 and 1797. Geoffroy mentioned the tick (found on an unidentified bat) as Acarus fuscus ovatus, pedibulus pallidis, vespertilionis, a taxon treated as Caris vespertilionis by Lamarck in the Histoire naturelle des animaux sans vertèbres (1839). The book of 
Table 2. List of bat parasites (Acari, Anoplura, Cestoda, Diptera, Hemiptera, Nematoda, Siphonaptera, Trematoda) and their hosts in France (including Corsica), based on the published literature, with reported synonyms. Authors are listed in the bibliography. See also the work entitled Les parasites métazoaires des Chiroptères de France (Acari, Anoplura, Cestoda, Diptera, Hemiptera, Nematoda, Siphonaptera, Trematoda) : contribution à un état des lieux bibliographique (1762-2018) et à l'établissement d'une liste nationale (2019). Invalid species are listed in brackets. Records marked with an exclamation mark (!) are invalid. Records marked with a question mark (?) are dubious. They may require further clarification.

\begin{tabular}{lll}
\hline Parasite species & $\begin{array}{c}\text { Number of } \\
\text { reported hosts }\end{array}$ & Bat species and citation \\
\hline
\end{tabular}

Acari

Acari, part 1/3: generally recognised taxa $(n=53)$, with their reported synonyms, and records without identification to species level $(n=6)$

Acanthophthirius (Myotimyobia) pantopus (Poppe et Trouessart, 1895)

Acanthophthirius (Acanthophthirius) poppei (Trouessart, 1895)

Alabidocarpus diceratops Lawrence, 1952

Alabidocarpus megalonyx (Trouessart, 1895)

Alabidocarpus minor (Rollinat \& Trouessart, 1897)

Argas pipistrellae (Audouin, 1832) = Argas vespertilionis (Latreille, 1796)

Argas transgariepinus White, $1846=$ Argas (Secretargas) transgariepinus White, 1846

Argas vespertilionis (Latreille, 1796)

Argas (Carios) vespertilionis (Latreille, 1802)

Argas (Secretargas) transgariepinus White, 1846

Chiroptella muscae (Oudemans, 1906) = Oudemansidium musca (Oudemans, 1906)

Calcarmyobia dusbabeki Uchikawa, 1985?

Calcarmyobia rhinolophia (Radford, 1940)!
1

1

1

1

1

11

(

Barbastella barbastellus (Schreber, 1774) [28]

Eptesicus serotinus (Schreber, 1774) [28, 38]

Miniopterus schreibersii (Natterer in Kuhl, 1817)? [85]

Myotis emarginatus (Geoffroy, 1806) [28]

Myotis myotis (Borkhausen, 1797) [28]

Myotis mystacinus (Leisler in Kuhl, 1817) [28]

Myotis sp. [171?, 242]

Pipistrellus kuhlii (Kuhl, 1817) [28, 166]

Pipistrellus nathusii (Keyserling \& Blasius, 1839) [28]

Pipistrellus pipistrellus (Schreber, 1774) [18, 28, 38, 67, 166]

Plecotus auritus (Linné, 1758) [28]

12 Eptesicus serotinus (Schreber, 1774) [31, 45, 86]

Hypsugo savii (Bonaparte, 1837) [45]

Myotis dasycneme (Boie, 1825) [31]

Myotis mystacinus (Leisler in Kuhl, 1817) [45]

Nyctalus leisleri (Kuhl, 1817) [45]

Pipistrellus kuhlii (Kuhl, 1817) [45]

Pipistrellus nathusii (Keyserling \& Blasius, 1839) [45]

Pipistrellus pipistrellus (Schreber, 1774) [31, 45, 177, 239]

Pipistrellus sp. [45]

Plecotus auritus (Linné, 1758) [45]

Plecotus austriacus Fischer, 1829 [31]

Rhinolophus ferrumequinum (Schreber, 1774) [45]

See also [5, 167-169]

Chiroptera gen. sp. [189]

Eptesicus serotinus (Schreber, 1774) [45, 189]

Hypsugo savii (Bonaparte, 1837) [45, 189]

Myotis emarginatus (Geoffroy, 1806) [45, 189]

Plecotus austriacus Fischer, 1829 [45, 189]

See also $[31,167]$
Miniopterus schreibersii (Natterer in Kuhl, 1817) [50, 51, 167] There is only one case. According to Lanza (1999) [publication $\mathrm{n}^{\circ} 167$ ], this field data published by Beron (1971) [publication $\left.\mathrm{n}^{\circ} 50\right]$ is doubtful. It could be Calcarmyobia dusbabeki Uchikawa, 1985 / Calcarmyobia rhinolophia (Radford, 1940). Miniopterus schreibersii (Natterer in Kuhl, 1817) [50, 51, 167] There is only one case. According to Lanza (1999) [publication $\mathrm{n}^{\circ} 167$ ], this field data published by Beron (1971) [publication $\left.\mathrm{n}^{\circ} 50\right]$ is doubtful. It could be Calcarmyobia dusbabeki Uchikawa, 1985 / Calcarmyobia rhinolophia (Radford, 1940). 
Table 2. (Continued)

\author{
Parasite species \\ Dermanyssus coriaceus $=$ Hirstionyssus arcuatus $($ Koch \\ 1839). See [11, 13, 221, 222] \\ Dermanyssus sp. \\ Dermanyssus pipistrellae (Gervais, 1841) = Hirstionyssus \\ arcuatus (Koch, 1839) \\ Diplostaspis daubentonii Kolenati, 1857 = Spinturnix \\ andegavinus (Deunff, 1977) according to Deunff (1977) \\ [76] \\ Diplostaspis stellata Kolenati, 1859 = Spinturnix \\ andegavinus (Deunff, 1977) according to Deunff (1977) \\ [76] \\ Eschatocephalus flavipes $($ Koch) = Ixodes \\ (Eschatocephalus) vespertilionis C.L. Koch, 1844 \\ Eschatocephalus vespertilionis C.L. Koch $=$ Ixodes \\ (Eschatocephalus) vespertilionis C.L. Koch, 1844 \\ Eschatocephalus vespertilionis $($ Koch 1844) = Ixodes \\ (Eschatocephalus) vespertilionis C.L. Koch, 1844
}

Eyndhovenia euryalis (G. Canestrini, 1885)

Eyndhovenia euryalis oudemansi (Eyndhoven, 1941)

Haemalastor vespertilionis $=$ Ixodes (Eschatocephalus) vespertilionis C.L. Koch, 1844

Hirstionyssus arcuatus (Koch, 1839)

Ichoronyssus diversipilis Vitzthum, 1920

Ichoronyssus scutatus (Kolenati, 1856)

Ichoronyssus spinosus (Oudemans) 1902)

Ichoronyssus spinosus (Oudemans, 1902) / Ichoronyssus scutatus (Kolenati, 1856)

Ichoronyssus $\mathrm{sp}$.

Ixodes chiropterum Babos et Janisch, 1958 = Ixodes (Eschatocephalus) simplex simplex Neumann, 1906

Ixodes gracilipes $=$ Ixodes (Eschatocephalus) vespertilionis C.L. Koch, 1844 ?

Ixodes hexagonus Leach, $1815=$ Pholeoixodes hexagonus (Leach, 1815)

Ixodes longipes (Lucas, 1872) = Ixodes (Eschatocephalus $)$ vespertilionis C.L. Koch, 1844

Ixodes (Pomerantzevella) simplex Neumann $1906=$ Ixodes (Eschatocephalus) simplex simplex Neumann, 1906

Ixodes pospelovae Emtchuck, 1955 = Ixodes

(Eschatocephalus) simplex simplex Neumann, 1906

Ixodes siculifer Mégnin, $1880=$ Ixodes (Eschatocephalus) vespertilionis C.L. Koch, 1844

Ixodes reduvius Geer $=$ Ixodes $($ Ixodes $)$ ricinus $($ Linnaeus, 1758)

Ixodes (Eschatocephalus) vespertilionis C.L. Koch, 1844
Number of

reported hosts

Bat species and citation

Myotis emarginatus (Geoffroy, 1806) [86]
Miniopterus schreibersii (Natterer in Kuhl, 1817) [76]

Myotis emarginatus (Geoffroy, 1806) [63, 121]

Rhinolophus euryale Blasius, 1853 [50, 63, 70, 76, 78, 105, 139, 167, 235]

Rhinolophus ferrumequinum (Schreber, 1774) [50, 63, 76, 78, 167]

Rhinolophus sp. [51, 76]

Rhinolophus ferrumequinum (Schreber, 1774) [18?, 105, 252]

Nyctalus noctula (Schreber, 1774) [11, 12, 103, 124, 127, 167, 177, 212, 221, 258, 259]

Miniopterus schreibersii (Natterer in Kuhl, 1817) [24]

Rhinolophus euryale Blasius, 1853 [50, 65, 167, 205, 212, 235, 246]

Miniopterus schreibersii (Natterer in Kuhl, 1817) [24, 70, 72]

Myotis myotis (Borkhausen, 1797)? [24, 70, 72]

Myotis myotis (Borkhausen, 1797)? [25]
Barbastella barbastellus (Schreber, 1774) [28, 32, 200]

Chiroptera gen. sp. [45, 69, 160, 177, 197-199, 248]

Eptesicus serotinus (Schreber, 1774) [28, 32, 166]

Miniopterus schreibersii (Natterer in Kuhl, 1817) ([6, 28, 176!] see

Beaucournu, 1966, p. 498; paper n`30 [200, 242])

Myotis bechsteinii (Leisler in Kuhl, 1817) [28, 32, 38

Myotis daubentonii (Leisler in Kuhl, 1817) [28, 32]

Myotis emarginatus (Geoffroy, 1806) [28, 32, 38, 136, 137, 177]

Myotis myotis (Borkhausen, 1797) [23, 28, 32, 38, 154, 166]

Myotis mystacinus (Leisler in Kuhl, 1817) [28, 32, 38, 85]

Myotis nattereri (Kuhl, 1817) [28, 32]

Pipistrellus kuhlii (Kuhl, 1817) [28] 
Table 2. (Continued)

\begin{tabular}{ll}
\hline Parasite species & $\begin{array}{c}\text { Number of } \\
\text { reported hosts }\end{array}$ \\
\hline
\end{tabular}

Pipistrellus nathusii (Keyserling \& Blasius, 1839) [28]

Pipistrellus pipistrellus (Schreber, 1774) [28, 86, 199]

Plecotus auritus (Linné, 1758) [28, 199]

Rhinolophus euryale Blasius, 1853 [28, 32, 85, 166]

Rhinolophus ferrumequinum (Schreber, 1774) [28, 32, 38, 45, 51, 58, 154, 177, 199, 200, 214, 215, 218, 229, 239]

Rhinolophus hipposideros (Bechstein, 1800) [23, 28, 32, 152, 154, 199, 200]

Rhinolophus sp. [5, 58, 126, 197]

Ixodes (Eschatocephalus) simplex simplex Neumann, 1906

See also [14, 31, 59, 143, 167, 201, 213]

Absence in Myotis blythii (Tomes, 1857) and M. blythii oxygnathus Monticelli, 1885 [32]

Absence in Pipistrellus kuhlii (Kuhl, 1817) [32]

Absence in Pipistrellus nathusii (Keyserling \& Blasius, 1839) [32]

Absence in Pipistrellus pipistrellus (Schreber, 1774) [32]

Absence in Plecotus auritus (Linné, 1758) [32]

Absence in Plecotus austriacus Fischer, 1829 [32]

Rhinolophus ferrumequinum (Schreber, 1774) ! [85]

Myotis mystacinus (Leisler in Kuhl, 1817) ! [85]

Myotis myotis (Borkhausen, 1797) ! [85]

Miniopterus schreibersii (Natterer in Kuhl, 1817) ([15, 31, 32, 45, 51, 143?, 166, 167, 176] see Beaucournu [1966; p. 498] paper n³0)

Labidocarpus megalonyx $($ Trouessart, 1895) = Alabidocarpus megalonyx (Trouessart, 1895)

Labidocarpus minor $($ Rollinat $\&$ Trouessart, 1897) $=$ Alabidocarpus minor (Rollinat \& Trouessart, 1897)

Labidocarpus (Labidocarpus) rollinati Trouessart, 1895

See also [213]

Myotis myotis (Borkhausen, 1797) [178]

Rhinolophus hipposideros (Bechstein, 1800) [178]

Leiognathus arcuatus $=$ Hirstionyssus arcuatus (Koch, 1839)

Leiognathus uncinatus = Macronyssus uncinatus (Canestrini, 1885)

Leptotrombidium (Leptotrombidium) russicum (Oudemans, 1902) = Leptotrombidium russicum (Oudemans, 1902)

Leptotrombidium russicum (Oudemans, 1902)

Rhinolophus ferrumequinum (Schreber, 1774) [167, 218, 232]

Rhinolophus hipposideros (Bechstein, 1800) [167, 218] see also [52, 191]

Leptus autumnalis $=$ Neotrombicula autumnalis (Shaw, 1790)

Liponyssus ellipticus = Macronyssus ellipticus (Kolenati, 1856)

Liponyssus euryale Canestrini $=$ Macronyssus rhinolophi (Oudemans, 1902)

Liponyssus longimanus $=$ Macronyssus longimanus (Kolenati)

Liponyssus spinosus Oudemans, $1902=$ Ichoronyssus scutatus (Kolenati, 1856)

Macronyssus ellipticus (Kolenati, 1856) !

Myotis daubentonii (Leisler in Kuhl, 1817) [122, 167, 177, 256] see also [10]

Rhinolophus hipposideros (Bechstein, 1800) [161] 
Table 2. (Continued)

\begin{tabular}{|c|c|c|}
\hline Parasite species & $\begin{array}{l}\text { Number of } \\
\text { reported hosts }\end{array}$ & Bat species and citation \\
\hline \multicolumn{3}{|l|}{$\begin{array}{l}\text { According to Radovsky [212], this observation published by } \\
\text { Husson \& Daum [152] is dubious. }\end{array}$} \\
\hline Macronyssus granulosus (Kolenati, 1856) & 2 & Miniopterus schreibersii (Natterer in Kuhl, 1817) [50, 167] \\
\hline \multicolumn{3}{|l|}{ Rhinolophus euryale Blasius, $1853[51,167]$} \\
\hline Macronyssus longimanus (Kolenati)! & 1 & Rhinolophus ferrumequinum (Schreber, 1774) ! [152, 177, 212] \\
\hline \multicolumn{3}{|l|}{$\begin{array}{l}\text { According to Radovsky [212], this observation published by } \\
\text { Husson \& Daum [152] is dubious. }\end{array}$} \\
\hline Macronyssus uncinatus (Canestrini, 1885) & 1 & Chiroptera gen. sp. $[160,177]$ \\
\hline \multirow[t]{2}{*}{ Macronyssus rhinolophi (Oudemans, 1902) } & 3 & Myotis myotis (Borkhausen, 1797)? [152?, 212?] \\
\hline & & $\begin{array}{l}\text { Rhinolophus ferrumequinum (Schreber, 1774)? [152?, 212?] } \\
\text { Rhinolophus hipposideros (Bechstein, 1800) [50, 152?, 167, 212?] }\end{array}$ \\
\hline \multicolumn{3}{|l|}{$\begin{array}{l}\text { Macronyssus spinosus }(\text { Oudemans, 1902) }=\text { Ichoronyssus } \\
\text { scutatus }(\text { Kolenati, 1856) }\end{array}$} \\
\hline \multicolumn{3}{|l|}{$\begin{array}{l}\text { Myobia pantopus }=\text { Acanthophthirius }(\text { Myotimyobia }) \\
\text { pantopus (Poppe et Trouessart, 1895) }\end{array}$} \\
\hline \multicolumn{3}{|l|}{$\begin{array}{l}\text { Myobia poppei }=\text { Acanthophthirius (Acanthophthirius) } \\
\text { poppei (Trouessart, 1895) }\end{array}$} \\
\hline \multirow[t]{2}{*}{ Neomyobia chiropteralis chiropteralis (Michael, 1884) } & 1 & $\begin{array}{l}\text { Rhinolophus hipposideros (Bechstein, 1800) [50, 52, 100, 167], see also } \\
\text { [114] }\end{array}$ \\
\hline & & See also [112] \\
\hline Neomyobia rollinati (Poppe, 1908) & 1 & Rhinolophus ferrumequinum (Schreber, 1774) [50, 52, 167] \\
\hline Neomyobia slovenica Dusbábek, 1969 & 1 & Rhinolophus euryale Blasius, 1853 [50-52, 167] \\
\hline Neomyobia sp. & 1 & Rhinolophus sp. [126] \\
\hline \multirow[t]{2}{*}{ Neotrombicula autumnalis (Shaw, 1790) } & 2 & Chiroptera gen. sp. [62] \\
\hline & & Myotis daubentonii (Leisler in Kuhl, 1817) $[8-10,122,167,177,256]$ \\
\hline Neotrombicula racovitzai Feider, 1970 & 1 & Eptesicus sp. $[5,118,167]$ \\
\hline $\begin{array}{l}\text { Neotrombicula vandeli Kolebinova \& Vercammen- } \\
\text { Grandjean, } 1971\end{array}$ & 1 & Rhinolophus euryale Blasius, 1853 [7, 162, 167] \\
\hline Notoedres (Notoedres) chiropteralis (Trouessart, 1896) & 2 & $\begin{array}{l}\text { Rhinolophus ferrumequinum (Schreber, 1774) }[106,109,167,218,250] \\
\text { Eptesicus serotinus (Schreber, 1774) }[48,106,167,250]\end{array}$ \\
\hline Nycteridocoptes eyndhoveni Fain, 1959 & 1 & Rhinolophus ferrumequinum (Schreber, 1774) [49, 107] \\
\hline Nycteridocoptes poppei Oudemans, 1898 & 3 & $\begin{array}{l}\text { Myotis blythii (Tomes, 1857) and M. blythii oxygnathus Monticelli, } \\
\quad 1885 \text { [113] } \\
\text { Myotis daubentonii (Leisler in Kuhl, 1817) [113] } \\
\text { Myotis myotis (Borkhausen, 1797) [24] }\end{array}$ \\
\hline Oudemansidium musca (Oudemans, 1906) & 1 & Myotis daubentonii (Leisler in Kuhl, 1817) $[122,167,177]$ \\
\hline \multicolumn{3}{|l|}{$\begin{array}{l}\text { Paraperiglischrus rhinolophinus (C.L. Koch) }= \\
\quad \text { Paraperiglischrus rhinolophinus (C.L. Koch, 1841) }\end{array}$} \\
\hline \multicolumn{3}{|l|}{$\begin{array}{l}\text { Paraperiglischrus rhinolophinus Koch, } 1841= \\
\quad \text { Paraperiglischrus rhinolophinus (C.L. Koch, 1841) }\end{array}$} \\
\hline Paraperiglischrus rhinolophinus (C.L. Koch, 1841) & 3 & $\begin{array}{l}\text { Rhinolophus euryale Blasius, } 1853 \text { [50, 76, 138, 167, 235] } \\
\text { Rhinolophus ferrumequinum (Schreber, 1774) [50, 78, 167, 235] } \\
\text { Rhinolophus hipposideros (Bechstein, 1800) [50, 138, 167, 223?, 235] }\end{array}$ \\
\hline \multicolumn{3}{|l|}{$\begin{array}{l}\text { Periglischrus interruptus (Kolenati, 1856) }= \\
\quad \text { Paraperiglischrus rhinolophinus (C.L. Koch, 1841) }\end{array}$} \\
\hline \multicolumn{3}{|l|}{$\begin{array}{l}\text { Prosopodectes chiropteralis }(\text { Trouessart, 1896) }=\text { Notoedres } \\
\quad \text { (Notoedres) chiropteralis }(\text { Trouessart, 1896) }\end{array}$} \\
\hline Psorergates rhinolophi Fain, 1959 & 1 & Rhinolophus ferrumequinum (Schreber, 1774) $[108,167]$ \\
\hline $\begin{array}{l}\text { Riedlinia (Riedlinia) petarberoni (Kolebinova \& } \\
\quad \text { Vercammen-Grandjean, 1970) }\end{array}$ & 1 & Rhinolophus euryale Blasius, 1853 [7, 51, 161, 167] \\
\hline \multicolumn{3}{|l|}{$\begin{array}{l}\text { Sarconissus vespertilionis }=\text { Ixodes (Eschatocephalus) } \\
\quad \text { vespertilionis } \text { C.L. Koch, } 1844\end{array}$} \\
\hline \multicolumn{3}{|l|}{$\begin{array}{l}\text { Sarcoptes chiropteralis Trouessart, } 1896=\text { Notoedres } \\
\quad(\text { Notoedres) chiropteralis (Trouessart, 1896) }\end{array}$} \\
\hline $\begin{array}{l}\text { Sasatrombicula (Sasatrombicula) bureschi Kolebinova \& } \\
\quad \text { Beron, } 1965\end{array}$ & 1 & Rhinolophus hipposideros (Bechstein, 1800) $[51,52,162,167]$ \\
\hline
\end{tabular}


Table 2. (Continued)

\begin{tabular}{lcc}
\hline Parasite species & $\begin{array}{c}\text { Number of } \\
\text { reported hosts }\end{array}$ & Bat species and citation \\
$\begin{array}{l}\text { Sasatrombicula (Sasatrombicula) hexasternalae } \\
\text { (Vercammen-Grandjean, 1963) }\end{array}$ & 4 & $\begin{array}{l}\text { Chiroptera gen. sp. [7] } \\
\text { Rhinolophus euryale Blasius, 1853 [51, 162, 167] } \\
\text { Rhinolophus ferrumequinum (Schreber, 1774) [51, 162, 167] } \\
\text { Rhinolophus hipposideros (Bechstein, 1800) [51, 162, 167] } \\
\text { Spyctalus noctula (Schreber, 1774) [recorded at Col de Bretolet, at the } \\
\text { border between France and Switzerland] [79] }\end{array}$ \\
Spinturnix andegavinus (Deunff, 1977) & 1 & Myotis daubentonii (Leisler in Kuhl, 1817) [76], see also [167]
\end{tabular}

This species is treated as Spinturnix andegavina Deunff, 1977 by some authors [167, 209, 225]. According to Sachanowicz et al. (publication $\mathrm{n}^{\circ} 223$; p. 49), this species "is a member of the myoti species group, which is actually recognised as also containing": $S$. dasycnemi (Kolenati, 1856), S. myoti (Kolenati, 1856), S. bechsteini Deunff et al. 2004, S. emarginata (Kolenati, 1856), and S. mystacina (Kolenati, 1857)". According to Lanza [167], this taxon is actually a synonym of Spinturnix daubentonii (Kolenati, 1857).

Spinturnix bechsteini (Deunff, Walter, Bellido et Volleth, 2004)

Spinturnix daubentonii (Kolenati, 1857). This species is treated as a synonym of Spinturnix andegavinus (Deunff, 1977 ) by some authors [167, 209, 225].

Spinturnix helvetiae (Deunff, Aellen \& Keller, 1986).

In the opinion of Uchikawa et al. (1994), this taxon is actually a subspecies of $S$. acuminata (C.L. Koch, 1836). See the article entitled "Contribution to the taxonomy of the genus Spinturnix (Acari: Spinturnicidae), with the erection of a new genus, Emballonuria" (Folia Parasitologica, 41 (4), p. 295).

Spinturnix emarginatus (Kolenati, 1856)

Spinturnix euryalis (G. Canestrini) = Eyndhovenia euryalis (G. Canestrini, 1885)

Spinturnix murinus $($ Walckenaer, 1847) = Spinturnix myoti (Kolenati, 1856)

Nyctalus noctula (Schreber, 1774) [recorded at Col de Bretolet, at the border between France and Switzerland] [79]

See also [77]

Spinturnix oudemansi van Eyndhoven, 1941 = Eyndhovenia euryalis (G. Canestrini, 1885)

See also Eyndhovenia euryalis oudemansi (Eyndhoven, 1941). 
Table 2. (Continued)

\begin{tabular}{|c|c|c|}
\hline Parasite species & $\begin{array}{l}\text { Number of } \\
\text { reported hosts }\end{array}$ & Bat species and citation \\
\hline Spinturnix plecotina (C.L. Koch, 1839) & 3 & $\begin{array}{l}\text { Chiroptera gen. sp. [7] } \\
\text { Plecotus auritus (Linné, 1758) [63, 76, 167] } \\
\text { Plecotus austriacus Fischer, } 1829[63,76,167]\end{array}$ \\
\hline \multicolumn{3}{|c|}{$\begin{array}{l}\text { Spinturnix plecotinus Koch, } 1839=\text { Spinturnix plecotina }(\mathrm{C} . \\
\quad \text { L. Koch, 1839) }\end{array}$} \\
\hline Spinturnix punctata (Sundevall, 1833) & 1 & Barbastella barbastellus (Schreber, 1774) [80, 167] \\
\hline Spinturnix psi (Kolenati, 1856) & 7 & $\begin{array}{l}\text { Chiroptera gen. sp. [7, 167, 210?, 223] } \\
\text { Eptesicus serotinus (Schreber, 1774) [48] } \\
\text { Miniopterus schreibersii (Natterer in Kuhl, 1817) [50, 63, 76, 139] } \\
\text { Myotis capaccinii (Bonaparte, 1837) [63, 76, 121, 139] } \\
\text { Myotis myotis (Borkhausen, 1797) [76] } \\
\text { Rhinolophus euryale Blasius, 1853 [76] } \\
\text { Rhinolophus ferrumequinum (Schreber, 1774) [139, 223?] }\end{array}$ \\
\hline & & See also [210] \\
\hline Spinturnix sp. & 1 & Myotis myotis (Borkhausen, 1797) [133] \\
\hline Steatonyssus sp. & 1 & Myotis myotis (Borkhausen, 1797) $[152,212]$ \\
\hline Steatonyssus spinosus Willmann, 1936 & 1 & Rhinolophus ferrumequinum (Schreber, 1774) [50, 51, 167] \\
\hline
\end{tabular}

Thrombicula autumnalis Shaw = Neotrombicula autumnalis (Shaw, 1790)

Thrombicula russica Oudemans = Leptotrombidium russicum (Oudemans, 1902)

Thrombicula russicum $=$ Leptotrombidium russicum (Oudemans, 1902)

Trombicula muscae = Oudemansidium musca (Oudemans, 1906)

Trombicula russicum = Leptotrombidium russicum (Oudemans, 1902)

Acari, part 2/3: invalid taxa $(n=22)$

[Acarus vespertilionis Hermann]

[Acarus vespertilionis Hermann, 1804 - la Mite de la chauve-souris]

[Argas caris]

$[$ Caris vespertilionis $=$ Argas pipistrellae $($ Audouin $) ?=$ Argas vespertilionis (Latreille, 1796)?]

[Dermanyssus coriaceus Gervais (=Hirstionyssus arcuatus (Koch, 1839)?)]

[Dermanyssus murinus (Lucas, 1840)]

[Dermanyssus vespertilionis Dugès, 1834]

[Gamassus dermanyssö̈des (Mégnin)]

[Gamasus pteroptoides (Mégnin)]

The species is recognised by Da Fonseca [72], Lanza [167] and Neumann (see A treatise on the parasites and parasitic diseases of the domesticated animals. Translation George Fleming, 2nd edition. Baillière Tindall, London, 1892, 574 p.; p. 99). Kramer treated the species as a synonym of Laelaps pteroptoides Mégnin (see the article entitled 'Ueber Milben', published in Archiv für Naturgeschichte, volume 52, issue 1, 1886, p. 251). Tiraboschi [244] treated the species as a synonym of Haemomyson musculi and Laelaps (Iphis) agilis Koch, Berlese. According to Rudnick [223], Gamasus pteroptoides (Mégnin) is not a species of the Spinturnicidae family.
Chiroptera gen. sp. [257, 258]

Nyctalus noctula (Schreber, 1774)? [90, 135]

Pipistrellus pipistrellus (Schreber, 1774) [191]

Nyctalus noctula (Schreber, 1774)? [168?, 171, 201]

Pipistrellus pipistrellus (Schreber, 1774)? [258]

See also [90]

Nyctalus noctula (Schreber, 1774) [11, 13, 103, 212, 222, 258, 259]

Myotis myotis (Borkhausen, 1797) [101, 104, 167, 183, 195]

Chiroptera gen. sp. [94]

Myotis mystacinus (Leisler in Kuhl, 1817) [248]

Pipistrellus pipistrellus (Schreber, 1774) [190] 
Table 2. (Continued)

\begin{tabular}{|c|c|c|}
\hline Parasite species & $\begin{array}{l}\text { Number of } \\
\text { reported hosts }\end{array}$ & Bat species and citation \\
\hline $\begin{array}{l}\text { [Haemomyson trouessarti (Mégnin) (=Leiognathus } \\
\text { arcuatus }=\text { Hirstionyssus arcuatus (Koch, 1839)? (See } \\
{[167,244], \text { p. 119) }}\end{array}$ & 3 & $\begin{array}{l}\text { Pipistrellus pipistrellus (Schreber, 1774) [191, 244] } \\
\text { Nyctalus noctula (Schreber, 1774) [191, 244] } \\
\text { [Vesperugo nystagmus] [191, 244]. This synonym is not mentioned by } \\
\quad \text { Didier and Rode in Les Mammifères de France }\end{array}$ \\
\hline $\begin{array}{l}\text { [Laelaps (Iphis) agilis Koch, Berlese = Gamasus } \\
\text { pteroptoides (Mégnin)? = Laelaps agilis C.L. Koch, } \\
\text { 1836?] }\end{array}$ & 1 & Pipistrellus pipistrellus (Schreber, 1774) ([244], see also [190]) \\
\hline $\begin{array}{l}\text { [Leiognathus armatus (=Hirstionyssus arcuatus (C.L. Koch, } \\
\text { 1839)?)] }\end{array}$ & 1 & Chiroptera gen. sp. [160, 177] \\
\hline $\begin{array}{l}\text { [Liponyssus arcuatus Koch (=Hirstionyssus arcuatus (C.L. } \\
\text { Koch, 1839) (pro parte) and Steatonyssus murinus } \\
\text { (Lucas, 1840) (pro parte)] }\end{array}$ & 1 & Rhinolophus ferrumequinum (Schreber, 1774) [200] \\
\hline $\begin{array}{l}\text { [Liponyssus decussatus (Kolenati) (=Caris decussata } \\
\quad \text { Kolenati, 1856?)] }\end{array}$ & 1 & Myotis myotis (Borkhausen, 1797) [23] \\
\hline [Liponyssus setosus (Kolenati)] & $2 ?$ & $\begin{array}{l}\text { Chiroptera gen. sp. [72] } \\
\text { Rhinolophus sp. [72] }\end{array}$ \\
\hline $\begin{array}{l}\text { [Pteroptus trouessarti Mégnin (=Haemomyson trouessarti } \\
\text { (Mégnin)?)] }\end{array}$ & 2 & $\begin{array}{l}\text { Myotis daubentonii (Leisler in Kuhl, 1817) }[177,248] \\
\text { Myotis mystacinus (Leisler in Kuhl, 1817) }[177,248]\end{array}$ \\
\hline [Pteroptus vespertilionis] & 3 & $\begin{array}{l}\text { Absence in Pipistrellus pipistrellus (Schreber, 1774) [67] } \\
\text { Barbastella barbastellus (Schreber, 1774) [67] } \\
\text { Chiroptera gen. sp. [160] } \\
\text { Nyctalus noctula (Schreber, 1774) [131] }\end{array}$ \\
\hline [Pteroptus vespertilionis Hermann] & 2 & $\begin{array}{l}\text { Rhinolophus ferrumequinum (Schreber, 1774) [215] } \\
\text { Pipistrellus pipistrellus (Schreber, 1774) [215] }\end{array}$ \\
\hline [Pteroptus vespertilionis (Dufour, 1832)] & 9 & $\begin{array}{l}\text { Barbastella barbastellus (Schreber, 1774) [127, 177] } \\
\text { Chiroptera gen. sp. [120, 127] } \\
\text { Eptesicus serotinus (Schreber, 1774) [190] } \\
\text { Miniopterus schreibersii (Natterer in Kuhl, 1817) [186] } \\
\text { Myotis myotis (Borkhausen, 1797) [90?, 92] } \\
\text { Myotis mystacinus (Leisler in Kuhl, 1817) [248] } \\
\text { Nyctalus noctula (Schreber, 1774) [131?, 177] } \\
\text { Rhinolophus ferrumequinum (Schreber, 1774) [200] } \\
\text { Rhinolophus hipposideros (Bechstein, 1800) [200] } \\
\text { See also [134, 193, 235] }\end{array}$ \\
\hline $\begin{array}{l}{[\text { Spinturnix vespertilionis] }} \\
\text { Myotis myotis (Borkhausen, 1797) [25] }\end{array}$ & 2 & Miniopterus schreibersii (Natterer in Kuhl, 1817) [24] \\
\hline [Spinturnix vespertilionis (C.L. Koch)] & 3 & $\begin{array}{l}\text { Miniopterus schreibersii (Natterer in Kuhl, 1817) [70] } \\
\text { Myotis myotis (Borkhausen, 1797) [70] } \\
\text { Plecotus auritus (Linné, 1758) [70] }\end{array}$ \\
\hline
\end{tabular}

Acari, part 3/3: taxa only noted as absent $(n=3)$

Ixodes canisuga Johnston, 1849

[Ixodes ricinus (Linné, 1746)]
Absence in Barbastella barbastellus (Schreber, 1774) [28] Absence in Eptesicus serotinus (Schreber, 1774) [28] Absence in Myotis bechsteinii (Leisler in Kuhl, 1817) [28] Absence in Myotis daubentonii (Leisler in Kuhl, 1817) [28] Absence in Myotis emarginatus (Geoffroy, 1806) [28] Absence in Myotis myotis (Borkhausen, 1797) [28]

Absence in Myotis mystacinus (Leisler in Kuhl, 1817) [28] Absence in Myotis nattereri (Kuhl, 1817) [28] Absence in Pipistrellus kuhlii (Kuhl, 1817) [28] Absence in Pipistrellus nathusii (Keyserling \& Blasius, 1839) [28] Absence in Pipistrellus pipistrellus (Schreber, 1774) [28] Absence in Plecotus auritus (Linné, 1758) [28] Absence in Rhinolophus euryale Blasius, 1853 [28] Absence in Rhinolophus ferrumequinum (Schreber, 1774) [28] Absence in Rhinolophus hipposideros (Bechstein, 1800) [28]

Absence in Barbastella barbastellus (Schreber, 1774) [28] Absence in Eptesicus serotinus (Schreber, 1774) [28] Absence in Myotis bechsteinii (Leisler in Kuhl, 1817) [28] Absence in Myotis daubentonii (Leisler in Kuhl, 1817) [28] Absence in Myotis emarginatus (Geoffroy, 1806) [28] 
Table 2. (Continued)

\begin{tabular}{lc}
\hline Parasite species & $\begin{array}{c}\text { Number of } \\
\text { reported hosts }\end{array}$ \\
\hline
\end{tabular}

Pholeoixodes hexagonus (Leach, 1815)

Araeopsylla gestroi (Rothschild, 1906)

Ceratopsyllus hexactenus $=$ Ischnopsyllus (Hexactenopsylla) hexactenus (Kolenati, 1856)

Ceratopsyllus octactenus = Ischnopsyllus (Ischnopsyllus) octactenus (Kolenati, 1856)

Ceratopsyllus pentactenus $=$ Nycteridopsylla pentactena (Kolenati, 1856)

Ceratopsyllus unipectinatus Taschenberg $=$ Rhinolophopsylla unipectinata unipectinata (Taschenberg, 1880)

Ischnopsyllus (Ischnopsyllus) elongatus (Curtis, 1832) Ischnopsyllus (Hexactenopsylla) hexactenus (Kolenati) $=$ Ischnopsyllus (Hexactenopsylla) hexactenus (Kolenati, 1856)

Ischnopsyllus (Hexactenopsylla) hexactenus (Kolenati, 1856)

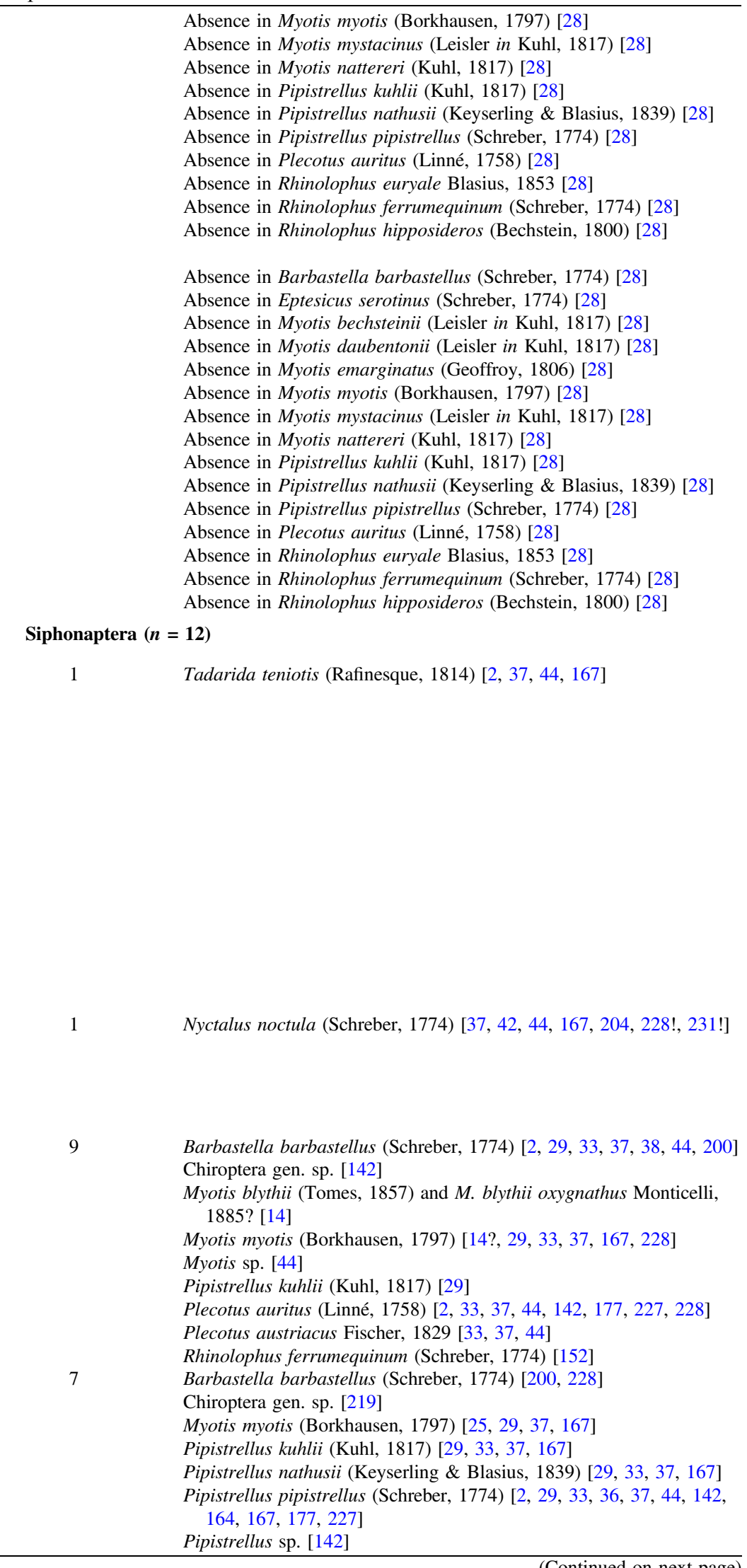

Absence in Myotis myotis (Borkhausen, 1797) [28]

Absence in Myotis mystacinus (Leisler in Kuhl, 1817) [28]

Absence in Myotis nattereri (Kuhl, 1817) [28]

Absence in Pipistrellus nathusii (Keyserling \& Blasius, 1839) [28

Absence in Pipistrellus pipistrellus (Schreber, 1774) [28]

(b)

Absence in Rhinolophus ferrumequinum (Schreber, 1774) [28]

ince in Rhinolophus hipposideros (Bechstein, 1800) [28]

(28]

Absence in Myotis emarginatus (Geoffroy, 1806) [28]

Absence in Myotis myotis (Borkhausen, 1797) [28]

Absence in Myotis nattereri (Kuhl, 1817) [28]

Absence in Pipistrellus kuhlii (Kuhl, 1817) [28

Absence in Plecotus auritus (Linné, 1758) [28]

Absence in Rhinolophus euryale Blasius, 1853 [28]

Absence in Rhinolophus ferrumequinum (Schreber, 1774) [28]

Absence in Rhinolophus hipposideros (Bechstein, 1800) [28]

Shonaptera $(n=12)$

Tadarida teniotis (Rafinesque, 1814) [2, 37, 44, 167]

Barbastella barbastellus (Schreber, 1774) [2, 29, 33, 37, 38, 44, 200] Chiroptera gen. sp. [142]

Myotis sp. [44]

Pipistrellus kuhlii (Kuhl, 1817) [29]

Myotis myotis (Borkhausen, 1797) [25, 29, 37, 167]

Pipistrellus kuhlii (Kuhl, 1817) [29, 33, 37, 167]

Pipistrellus nathusii (Keyserling \& Blasius, 1839)

164, 167, 177, 227]

(Continued on next page) 
Table 2. (Continued)

\begin{tabular}{|c|c|c|}
\hline Parasite species & $\begin{array}{l}\text { Number of } \\
\text { reported hosts }\end{array}$ & Bat species and citation \\
\hline \multicolumn{3}{|l|}{$\begin{array}{l}\text { Ischnopsyllus (Ischnopsyllus) intermedius (Rothschild) }= \\
\text { Ischnopsyllus (Ischnopsyllus) intermedius (Rothschild, } \\
\text { 1898) }\end{array}$} \\
\hline $\begin{array}{l}\text { Ischnopsyllus (Ischnopsyllus) intermedius (Rothschild, } \\
\text { 1898) }\end{array}$ & 6 & $\begin{array}{l}\text { Chiroptera gen. sp. [44] } \\
\text { Eptesicus serotinus (Schreber, 1774) [29, 33, 37, 38, 142, 167, 227] } \\
\text { Myotis myotis (Borkhausen, 1797) [2, 29, 33, 37, 142, 167] } \\
\text { Nyctalus leisleri (Kuhl, 1817) [44] } \\
\text { Nyctalus noctula (Schreber, 1774) [3, 44] } \\
\text { Rhinolophus ferrumequinum (Schreber, 1774) [29] }\end{array}$ \\
\hline Ischnopsyllus (Ischnopsyllus) simplex Rothschild, 1906 & 8 & $\begin{array}{l}\text { Barbastella barbastellus (Schreber, 1774) [29, 37] } \\
\text { Myotis daubentonii (Leisler in Kuhl, 1817) [44] } \\
\text { Myotis emarginatus (Geoffroy, 1806) [29, 37, 142] } \\
\text { Myotis myotis (Borkhausen, 1797) [29, 37, 85?] } \\
\text { Myotis mystacinus (Leisler in Kuhl, 1817) [2, 29, 33, 37, 44, 85?, 142, } \\
\quad \text { 156] } \\
\text { Myotis nattereri (Kuhl, 1817) [29, 33, 37, 44, 85?, 142] } \\
\text { Plecotus auritus (Linné, 1758)? [29, 37] } \\
\text { Rhinolophus ferrumequinum (Schreber, 1774)? [152, 177] } \\
\text { See also [167] }\end{array}$ \\
\hline \multicolumn{3}{|l|}{$\begin{array}{l}\text { Ischnopsyllus (Ischnopsyllus) variabilis Wagner = } \\
\quad \text { Ischnopsyllus (Ischnopsyllus) variabilis (Wagner, 1898) }\end{array}$} \\
\hline Ischnopsyllus (Ischnopsyllus) variabilis (Wagner, 1898) & 6 & $\begin{array}{l}\text { Myotis daubentonii (Leisler in Kuhl, 1817) [44] } \\
\text { Nyctalus noctula (Schreber, 1774) [37] } \\
\text { Pipistrellus kuhlii (Kuhl, 1817) [37, 44, 142] } \\
\text { Pipistrellus nathusii (Keyserling \& Blasius, 1839) [2, 29, 33, 37, 44] } \\
\text { Pipistrellus pipistrellus (Schreber, 1774) [33, 37, 85] } \\
\text { Plecotus auritus (Linné, 1758) ! [85] }\end{array}$ \\
\hline Nycteridopsylla ancyluris ancyluris Jordan, 1942 & 1 & Pipistrellus pipistrellus (Schreber, 1774) $[33,37,44,142,157,164]$ \\
\hline Nycteridopsylla dictena (Kolenati, 1856)! & 1 & $\begin{array}{l}\text { Chiroptera gen. sp.! Doubtful : see Beaucournu et Launay } \\
\left.\left(1990 ; \text { p. } 314 \text { [paper } n^{\circ} 37\right]\right)[37,146,167,227]\end{array}$ \\
\hline
\end{tabular}

Nycteridopsylla dictenus (Kolenati, 1857) = Nycteridopsylla dictena (Kolenati, 1856)

Nycteridopsylla (Dinycteropsylla) dictena (Kolenati, 1856) = Nycteridopsylla dictena (Kolenati, 1856)

Nycteridopsylla eusarca Dampf, 1908

Nycteridopsylla longiceps Rothschild, 1908

Nycteridopsylla pentactena (Kolenati, 1856)
Nyctalus noctula (Schreber, 1774) [37, 42]

Barbastella barbastellus (Schreber, 1774) [29, 33, 37, 167]

Myotis capaccinii (Bonaparte, 1837) [37, 164, 167]

Myotis daubentonii (Leisler in Kuhl, 1817) [29, 37, 167]

Myotis myotis (Borkhausen, 1797) ! [228]

Myotis mystacinus (Leisler in Kuhl, 1817) [29, 37, 38, 167]

Myotis nattereri (Kuhl, 1817) [29, 37, 167]

Pipistrellus kuhlii (Kuhl, 1817) [37, 44, 156]

Pipistrellus nathusii (Keyserling \& Blasius, 1839) [37, 44, 167]

Pipistrellus pipistrellus (Schreber, 1774) [2, 29, 33, 37, 85, 167]

Plecotus auritus (Linné, 1758) [33, 37, 85, 167, 227]

Barbastella barbastellus (Schreber, 1774) [2, 29, 33, 37, 44, 167, 200]

Chiroptera gen. sp. [187]

Eptesicus serotinus (Schreber, 1774) [29, 33, 37, 164, 167]

Miniopterus schreibersii (Natterer in Kuhl, 1817) [37, 164, 167]

Myotis capaccinii (Bonaparte, 1837) [37, 164, 167]

Myotis myotis (Borkhausen, 1797) [29, 37, 167]

Myotis mystacinus (Leisler in Kuhl, 1817) [29, 37, 167]

Myotis nattereri (Kuhl, 1817) [29, 37, 167]

Pipistrellus nathusii (Keyserling \& Blasius, 1839) [44]

Pipistrellus pipistrellus (Schreber, 1774) [29, 37, 38, 167]

Plecotus auritus (Linné, 1758) [29, 33, 37, 167]

Plecotus austriacus Fischer, 1829 [33, 37, 167]

Rhinolophus ferrumequinum (Schreber, 1774) [29, 37, 152, 164, 167, 177]

See also [217] 
Table 2. (Continued)

Parasite species
Pulex pungens Walckenaer (1802) (in Faune parisienne) =
Nycteridopsylla eusarca Dampf, 1908
[Pulex vespertilionis Dugès, 1832]
See also [90]
Rhinolophopsylla unipectinata unipectinata (Taschenberg,
1880)
Basilia italica Theodor = Basilia (Basilia) italica Theodor,
1954
Basilia nana Theodor = Basilia (Basilia) nana Theodor \&
Moscona, 1954
Basilia nattereri $($ Kolenati) = Basilia (Basilia) nattereri
(Kolenati, 1857)

Basilia (Basilia) italica Theodor, 1954

Basilia (Basilia) mediterranea Hůrka, 1970

Basilia (Basilia) nana Theodor \& Moscona, 1954

Basilia (Basilia) nattereri (Kolenati, 1857)

Brachytarsina flavipennis Macquart, 1851

Celeripes biarticulata $=$ Phthiridium biarticulatum Hermann, 1804

Listropodia pedicularia $=$ Nycteribia $($ Nycteribia $)$ pedicularia Latreille, 1805

Listropodia Schmidli $=$ Nycteribia $($ Nycteribia $)$ schmidlii Schiner, 1853

Nycteribia (Achrocholidia) vexata Westwood = Nycteribia (Achrocholidia) vexata Westwood, 1835

Nycteribia (Achrocholidia) vexata Westwood, 1835
Number of reported hosts

Bat species and citation
$1 \quad$ Myotis myotis (Borkhausen, 1797) [95]

$4 \quad$ Rhinolophus euryale Blasius, 1853 [2, 29, 33, 142, 167] Rhinolophus ferrumequinum (Schreber, 1774) [2, 33, 38, 44, 142, 152, 156, 164, 167, 177, 227, 242]

Rhinolophus hipposideros (Bechstein, 1800) [2, 86, 142, 167, 200, 228] Rhinolophus sp. [44]

Diptera $(n=13)$
$2 \quad$ Myotis emarginatus (Geoffroy, 1806) [4, 34]

Myotis mystacinus (Leisler in Kuhl, 1817) [34, 35, 43, 237]

See also [28, 150, 167]

$4 \quad$ Eptesicus serotinus (Schreber, 1774) [43, 154, 237]

Hypsugo savii (Bonaparte, 1837) [41, 167, 237]

Pipistrellus pipistrellus (Schreber, 1774) [40, 43, 167]

Pipistrellus sp. [41]

Myotis bechsteinii (Leisler in Kuhl, 1817) [4, 28, 30, 38, 237]

Myotis emarginatus (Geoffroy, 1806) [43, 237]

Myotis mystacinus (Leisler in Kuhl, 1817) [241]

Myotis nattereri (Kuhl, 1817) [4, 30, 237]

Plecotus auritus (Linné, 1758) [28, 237]

See also [150, 167]

Eptesicus serotinus (Schreber, 1774) [241]

Myotis daubentonii (Leisler in Kuhl, 1817) [187]

Myotis nattereri (Kuhl, 1817) [4, 28, 30, 43, 187]

Plecotus auritus (Linné, 1758) [28, 187]

See also [150, 167, 237]

1

Rhinolophus ferrumequinum (Schreber, 1774) [26, 40, 43, 117, 155, 237]
Myotis emarginatus (Geoffroy, 1806) ! [23]

Rhinolophus euryale Blasius, 1853 ! [85]

Rhinolophus ferrumequinum (Schreber, 1774) ! [85]

Rhinolophus hipposideros (Bechstein, 1800) ! [85]

Miniopterus schreibersii (Natterer in Kuhl, 1817) [1, 85, 167]

Myotis blythii (Tomes, 1857) and M. blythii oxygnathus Monticelli, 1885 [30]

Myotis myotis (Borkhausen, 1797) [1, 23, 25, 28, 30, 38, 167]

Myotis myotis (Borkhausen, 1797)/Miniopterus schreibersii (Natterer in

Kuhl, 1817) [43]

See also $[117,150,237]$ 
Table 2. (Continued)

\author{
Parasite species \\ Nycteribia (Celeripes) biarticulata Hermann = Phthiridium \\ biarticulatum Hermann, 1804 \\ Nycteribia biarticulata $=$ Phthiridium biarticulatum \\ Hermann, 1804 \\ Nycteribia blasii $($ Kolenati) $=$ Nycteribia $($ Nycteribia $)$ \\ kolenatii Theodor \& Moscona, 1954 \\ Nycteribia Celeripes Bi-Articulata $=$ Phthiridium \\ biarticulatum Hermann, 1804 \\ Nycteribia (Celeripes) biarticulata Hermann $1804=$ \\ Phthiridium biarticulatum Hermann, 1804 \\ Nycteribia Hermanni Leach $=$ Phthiridium biarticulatum \\ Hermann, 1804 \\ Nycteribia kolenatii Theodor $=$ Nycteribia $($ Nycteribia $)$ \\ kolenatii Theodor \& Moscona, 1954 \\ Nycteribia latreilli Leach $=$ Nycteribia $($ Nycteribia $)$ latreillii \\ (Leach, 1817) \\ Nycteribia (Listropodia) Schmidti Schiner $=$ Nycteribia \\ (Nycteribia) schmidlii Schiner, 1853 \\ Nycteribia pedicularia $=$ Nycteribia $($ Nycteribia $)$ pedicularia \\ Latreille, 1805) \\ Nycteribia schmidli $=$ Nycteribia $($ Nycteribia $)$ schmidlii \\ Schiner, 1853 \\ Nycteribia vespertilionis Dufour, $1831=$ Penicillidia \\ (Penicillidia) dufourii (Westwood, 1834) \\ Nycteribia vexata $=$ Nycteribia $($ Achrocholidia) vexata \\ Westwood, 1835
}

Nycteribia (Nycteribia) kolenatii Theodor \& Moscona, 1954

Nycteribia (Nycteribia) latreillii (Leach, 1817)

Nycteribia (Nycteribia) pedicularia Latreille, 1805
Number of reported hosts
Bat species and citation 
Table 2. (Continued)

\begin{tabular}{lc}
\hline Parasite species & $\begin{array}{c}\text { Number of } \\
\text { reported hosts }\end{array}$ \\
\hline
\end{tabular}

Myotis blythii (Tomes, 1857) and M. blythii oxygnathus Monticelli, $1885[115,154,167]$

Myotis capaccinii (Bonaparte, 1837) [1, 30, 85?]

Myotis myotis (Borkhausen, 1797) [25?, 186]

See also $[28,85,117,149,150,177,189 ?, 206 ?, 237]$. About old records of Nycteribia pedicularia see Szentiványi et al., 2016; p. 102 [237]

Miniopterus schreibersii (Natterer in Kuhl, 1817) [1, 24, 28, 30, 43 , $115,149,154,167,242]$ see also $[117,150,237]$

Miniopterus schreibersii (Natterer in Kuhl, 1817)/ Myotis myotis (Borkhausen, 1797) [30]

Myotis blythii (Tomes, 1857) and M. blythii oxygnathus Monticelli, 1885 [30]

Myotis daubentonii (Leisler in Kuhl, 1817) [147]

Myotis myotis (Borkhausen, 1797) [1, 30]

Pipistrellus pipistrellus (Schreber, 1774) [147]

Rhinolophus ferrumequinum (Schreber, 1774) [1]

Rhinolophus mehelyi Matschie, 1901 [147]

Myotis daubentonii (Leisler in Kuhl, 1817) [247]

Pipistrellus pipistrellus (Schreber, 1774) [214, 215]

Rhinolophus ferrumequinum (Schreber, 1774) [73]

See also [74]

Nycteribia (Stylidia) biarticulata Hermann $=$ Phthiridium biarticulatum Hermann, 1804

Nycteribia $($ Stylidia $)$ biarticulata $=$ Phthiridium biarticulatum Hermann, 1804

[Nycteribia eparticulata]

[Nycteribia frauenfeldi (Kolenati)] = Penicillidia

(Penicillidia) dufourii (Westwood, 1834)

[Nycteribia vespertilionis Latreille]

Nyctéribie du Vespertilion

[Nycteribia vespertilionis Meig.]

Nycteribosca kollari Frauenfeld, 1855 = Brachytarsina flavipennis Macquart, 1851

Complex of species Nycteribia kolenatiil latreilliilpedicularia

Penicillidia conspicua Speiser $=$ Penicillidia (Neopenicillidia) conspicua Speiser, 1901

Penicillidia d. dufourii $($ Westwood, 1834) = Penicillidia (Penicillidia) dufourii (Westwood, 1834)

Penicillidia dufouri Westwood $=$ Penicillidia $($ Penicillidia $)$ dufourii (Westwood, 1834)

Penicillidia (Neopenicillidia) conspicua Speiser, 1901

Miniopterus schreibersii (Natterer in Kuhl, 1817) [1, 28, 30, 115, 154, $167,242]$, see also [117, 150, 237]

Myotis myotis (Borkhausen, 1797) [25]

Chiroptera gen. sp. [43]

Miniopterus schreibersii (Natterer in Kuhl, 1817) [1, 24, 30, 237]

Myotis blythii (Tomes, 1857) and M. blythii oxygnathus Monticelli, 1885 [14?, 43, 115?, 154?, 237]

Myotis capaccinii (Bonaparte, 1837) [1, 237]

Myotis myotis (Borkhausen, 1797) [1, 14?, 22, 28, 30, 43, 90? 91?, 186?, 237, 242?]

Pipistrellus sp. [53]

Rhinolophus ferrumequinum (Schreber, 1774)? [200]

See also [93?, 117?, 150, 167, 260?] 
Table 2. (Continued)

\begin{tabular}{lll}
\hline Parasite species & $\begin{array}{c}\text { Number of } \\
\text { reported hosts }\end{array}$ & Bat species and citation \\
\hline
\end{tabular}

Phthiridium biarticulatum Hermann, 1804

Penicillidia (Penicillidia) monoceros Speiser, 1900

Cimex dissimilis (Horváth, 1910)

Cimex lectularius Merrett $1667=$ Cimex lectularius Linnaeus, 1758

Cimex lectularius Linnaeus, 1758

Cimex pipistrelli Jenins, 1839

Cimex stadleri Horváth 1935 = Cimex dissimilis (Horváth, 1910)

Cimex sp.

Polyplax serrata (Burmeister, 1839) !

Hymenolepis acuta = Vampirolepis acuta (Rudolphi, 1819) Hymenolepis balsaci $=$ Vampirolepis balsaci $($ Joyeux \& Baer, 1934)

Hymenolepis grisea $=$ Milina grisea van Beneden, 1873

Hymenolepis grisea (P.J. Van Beneden 1873) = Milina grisea van Beneden, 1873

Hymenolepis sp. = Vampirolepis species

Milina grisea van Beneden, 1873
Chiroptera gen. sp.? [173?, 204?]

Miniopterus schreibersii (Natterer in Kuhl, 1817)? [85, 126, 237]

Plecotus auritus (Linné, 1758) [85, 86, 237]

Rhinolophus euryale Blasius, 1853 [28, 30, 38, 115, 167, 242]

Rhinolophus ferrumequinum (Schreber, 1774) [1, 28, 30, 43, 85, 115, 126?, 135?, 151, 152, 154, 156, 175?, 177, 237, 242]

Rhinolophus hipposideros (Bechstein, 1800) [115, 126?, 135?, 167, 237]

See also $[47,116,117,150]$

Absence in Barbastella barbastellus (Schreber, 1774) [28]

Absence in Eptesicus serotinus (Schreber, 1774) [28]

Absence in Myotis bechsteinii (Leisler in Kuhl, 1817) [28]

Absence in Myotis daubentonii (Leisler in Kuhl, 1817) [28]

Absence in Myotis emarginatus (Geoffroy, 1806) [28]

Absence in Myotis myotis (Borkhausen, 1797) [28]

Absence in Myotis mystacinus (Leisler in Kuhl, 1817) [28]

Absence in Myotis nattereri (Kuhl, 1817) [28]

Absence in Pipistrellus kuhlii (Kuhl, 1817) [28]

Absence in Pipistrellus nathusii (Keyserling \& Blasius, 1839) [28]

Absence in Pipistrellus pipistrellus (Schreber, 1774) [28]

Absence in Plecotus auritus (Linné, 1758) [28]

Absence in Rhinolophus euryale Blasius, 1853 [28]

Absence in Rhinolophus ferrumequinum (Schreber, 1774) [28]

Absence in Rhinolophus hipposideros (Bechstein, 1800) [28]

Hemiptera $(n=3)$

4 (5?) Chiroptera gen. sp. [207]

Myotis emarginatus (Geoffroy, 1806) [254]

Myotis myotis (Borkhausen, 1797)? [177]

Myotis sp. [253]

Rhinolophus ferrumequinum (Schreber, 1774) [254]

See also [167, 236]

1 (5?) Chiroptera gen. sp. [28, 35]

Myotis emarginatus (Geoffroy, 1806)? [253, 254]

Myotis myotis (Borkhausen, 1797)? [253, 254]

Rhinolophus euryale Blasius, 1853? [253, 254]

Rhinolophus ferrumequinum (Schreber, 1774)? [253, 254]

See also [167, 236]

1 Chiroptera gen. sp. [28, 236]

$1 \quad$ Chiroptera gen. sp. [28, 177]

Anoplura $(n=1)$

1 ! Rhinolophus hipposideros (Bechstein, 1800) ! [17, 177, 216]

Cestoda $(n=3)$

4 (5?) Miniopterus schreibersii (Natterer in Kuhl, 1817) [68, 86, 167]

Myotis emarginatus (Geoffroy, 1806) [68, 86, 167]

Myotis myotis (Borkhausen, 1797) [68, 159, 167]

Rhinolophus ferrumequinum (Schreber, 1774) [68, 71, 159, 196, 243]

Rhinolophus ferrumequinum or R. hipposideros or M. schreibersii [200] 
Table 2. (Continued)

\begin{tabular}{lcc}
\hline Parasite species & $\begin{array}{c}\text { Number of } \\
\text { reported hosts }\end{array}$ & Bat species and citation \\
\hline $\begin{array}{l}\text { Vampirolepis acuta (Rudolphi, 1819) } \\
\text { Vampirolepis balsaci (Joyeux \& Baer, 1934) }\end{array}$ & 1 & $\begin{array}{c}\text { Eptesicus serotinus } \text { (Schreber, 1774) [158, 159, 177, 196, 255] } \\
\text { Eptesicus serotinus (Schreber, 1774) [158, 159, 167, 177, 180, 196, } \\
\text { 255] } \\
\text { Myotis bechsteinii (Leisler in Kuhl, 1817) [159, 167, 177, 180, 196, } \\
\text { 255] }\end{array}$ \\
$\begin{array}{l}\text { Vampirolepis sp. / Milina sp. } \\
\text { Eptesicus serotinus } \text { (Schreber, 1774) [86] } \\
\text { Plecotus auritus (Linné, 1758) [86] }\end{array}$ & 2 & Trematoda (n = 15) \\
Allassogonoporus amphoraeformis (Mödlinger, 1930) & Myotis daubentonii (Leisler in Kuhl, 1817) [89, 167]
\end{tabular}

Distoma ascidoüdes Van Beneden, 1873 = Prosthodendrium (Prosthodendrium) chilostomum (Mehlis, 1831)

Distoma heteroptum (Dujardin, 1845) = Pycnoporus heteroporus (Dujardin, 1845)

Distoma lagena Brandes, 1888 = Lecithodendrium granulosum Looss, 1907

Lecithodendrium granulosum Looss, 1907

Lecithodendrium linstowi $\mathrm{R} . \mathrm{Ph}$ Dollfus $1931=$ Lecithodendrium linstowi Dollfus, 1931

Eptesicus serotinus (Schreber, 1774) [68, 88, 167] Miniopterus schreibersii (Natterer in Kuhl, 1817) [68, 167]

Myotis capaccinii (Bonaparte, 1837) [68, 167]

Myotis emarginatus (Geoffroy, 1806) [68, 167]

Myotis myotis (Borkhausen, 1797) [68, 167]

Pipistrellus kuhlii (Kuhl, 1817) [86, 167]

Pipistrellus pipistrellus (Schreber, 1774) [68, 167]

Plecotus austriacus Fischer, 1829 [68, 167]

Rhinolophus euryale Blasius, 1853 [68, 167]

Rhinolophus ferrumequinum (Schreber, 1774) [68, 167, 243]

Lecithodendrium modlingeri $($ Pande, 1935) =

Lecithodendrium moedlingeri (Pande, 1935)

Lecithodendrium moedlingeri (Pande, 1935)

Lecithodendrium sp.

Lepoderma vespertilionis (Müller) = Plagiorchis vespertilionis (O.F. Müller, 1784)

Limatulum duboisi Hurkova 1961 = Parabascus duboisi (Hurkova, 1961)

Miniopterus schreibersii (Natterer in Kuhl, 1817) [68, 167, 188] Rhinolophus ferrumequinum (Schreber, 1774) [68, 71, 88, 167, 188, 243]

Rhinolophus hipposideros (Bechstein, 1800) [68, 167, 188]

See also [202]

Myotis daubentonii (Leisler in Kuhl, 1817) [89, 167]

Miniopterus schreibersii (Natterer in Kuhl, 1817) [68, 167]

Plecotus austriacus Fischer, 1829 [68, 167]

Rhinolophus euryale Blasius, 1853 [68, 167]

Rhinolophus ferrumequinum (Schreber, 1774) [68, 167]

Pipistrellus pipistrellus (Schreber, 1774) [60, 68, 167]

Miniopterus schreibersii (Natterer in Kuhl, 1817) [200]

Rhinolophus ferrumequinum (Schreber, 1774) [200] Rhinolophus hipposideros (Bechstein, 1800)? [200] 
Table 2. (Continued)

\begin{tabular}{lcc}
\hline Parasite species & $\begin{array}{c}\text { Number of } \\
\text { reported hosts }\end{array}$ & Bat species and citation \\
\hline
\end{tabular}

Plagiorchis vespertilionis (O.F. Müller, 1784)

Eptesicus serotinus (Schreber, 1774) [68, 167]

Miniopterus schreibersii (Natterer in Kuhl, 1817) [68, 167, 200, 235]

Myotis capaccinii (Bonaparte, 1837) [68, 167]

Myotis daubentonii (Leisler in Kuhl, 1817) [89, 167]

Myotis emarginatus (Geoffroy, 1806) [68, 86, 167]

Myotis myotis (Borkhausen, 1797) [86]

Pipistrellus pipistrellus (Schreber, 1774) [68, 86, 167]

Plecotus auritus (Linné, 1758) [86, 167]

Rhinolophus euryale Blasius, $1853[68,167]$

Rhinolophus ferrumequinum (Schreber, 1774) [68, 71, 86-88, 167, 200, 243]

Prosthodendrium carolinum J. Hurkova, $1959=$

Rhinolophus hipposideros (Bechstein, 1800) [68, 86, 167, 200]

Prosthodendrium (Prosthodendrium) carolinum

Hurková, 1959

Prosthodendrium chilostoma $($ Mehlis, 1831) $=$

Prosthodendrium (Prosthodendrium) chilostomum

(Mehlis, 1831)

Prosthodendrium chilostomum $($ Mehlis $)=$ Prosthodendrium

(Prosthodendrium) chilostomum (Mehlis, 1831)

Prosthodendrium longiforme $($ Bhalerao, 1926) $=$

Prosthodendrium (Prosthodendrium) longiforme

(Bhalerao, 1926)

Prosthodendrium (Prosthodendrium) carolinum Hurková, 1959

Prosthodendrium (Prosthodendrium) chilostomum (Mehlis, 1831)

Prosthodendrium (Prosthodendrium) hurkovaae Dubois, 1960

Prosthodendrium (Prosthodendrium) longiforme (Bhalerao, 1926)

Prosthodendrium parvouterus (Bhalerao, 1926)

Prosthodendrium sp.

Pycnoporus heteroporus (Dujardin, 1845)

Histiostrongylus tipula (van Beneden, 1873) = Molinostrongylus tipula (Beneden, 1873)

Litomosa beaucournui Bain, 1967 = Litomosa ottavianii Lagrange \& Bettini, 1948

Litomosa desportesi Bain, 1967 = Litomosa dogieli

Bogdanov \& Vladimirov, 1956

Litomosa dogieli Bogdanov \& Vladimirov, 1956

Litomosa filaria P.J. Van Beneden 1873 = Litomosa filaria (Beneden, 1873)

Litomosa filaria $($ van Beneden $)=$ Litomosa filaria $($ Beneden, 1873)

Litomosa filaria (Beneden, 1873)
$2 \quad$ Myotis emarginatus (Geoffroy, 1806) [19] Myotis myotis (Borkhausen, 1797) [19]
Nematoda $(n=13)$

Rhinolophus ferrumequinum (Schreber, 1774) [167, 243]

Miniopterus schreibersii (Natterer in Kuhl, 1817) [68, 167, 200, 235] Myotis myotis (Borkhausen, 1797) [57, 61, 167]

Rhinolophus ferrumequinum (Schreber, 1774) [200, 243]

Rhinolophus hipposideros (Bechstein, 1800) [86, 167, 200, 235]

Myotis daubentonii (Leisler in Kuhl, 1817) [89]

Eptesicus serotinus (Schreber, 1774) [88, 167]

Rhinolophus ferrumequinum (Schreber, 1774) [167, 243]

Miniopterus schreibersii (Natterer in Kuhl, 1817) [68, 167]

Plecotus auritus (Linné, 1758) [86]

Pipistrellus kuhlii (Kuhl, 1817) [86]

Pipistrellus pipistrellus (Schreber, 1774) [57, 61, 68, 97, 163, 167, 235]
Myotis emarginatus (Geoffroy, 1806) [86, 167]

Myotis myotis (Borkhausen, 1797) [75, 86, 167] Plecotus auritus (Linné, 1758) [19, 167] 
Table 2. (Continued)

\begin{tabular}{lcl}
\hline Parasite species & $\begin{array}{c}\text { Number of } \\
\text { reported hosts }\end{array}$ & \multicolumn{1}{c}{ Bat species and citation } \\
\hline $\begin{array}{l}\text { Litomosa ottavianii Lagrange \& Bettini, 1948 } \\
\text { Molinostrongylus alatus (Ortlepp, 1932)? }\end{array}$ & 5 & $\begin{array}{l}\text { Miniopterus schreibersii (Natterer in Kuhl, 1817) [19, 167] } \\
\text { Myotis emarginatus (Geoffroy, 1806) [68, 167] } \\
\text { Rhinolophus euryale Blasius, 1853 [68, 167] } \\
\text { Rhinolophus ferrumequinum (Schreber, 1774) [19, 167] } \\
\text { Rhinolophus sp. [19, 167] }\end{array}$ \\
$\begin{array}{l}\text { Molinostrongylus tipula (Beneden, 1873) } \\
\text { Molinostrongylus ornatus (Monnig, 1927) }\end{array}$ & 1 167] \\
$\begin{array}{l}\text { Molinostrongylus panousei Dollfus, 1954 } \\
\text { Ophiostoma mucronatum (Rudolphi, 1809) }=\text { Seuratum }\end{array}$ & 2 & $\begin{array}{l}\text { Miniopterus schreibersii (Natterer in Kuhl, 1817) [200] } \\
\text { Myotis myotis (Borkhausen, 1797) [56] } \\
\text { Myotis myotis (Borkhausen, 1797) [68] } \\
\text { Miniopterus schreibersii (Natterer in Kuhl, 1817) [99, 167] }\end{array}$
\end{tabular}

Ophiostoma mucronatum (Rudolphi, 1809) = Seuratum mucronatum (Rudolphi, 1809)

Pterygodermatites (Neopaucipectines) bovieri (Blanchard, 1886)

Rictularia bovieri $=$ Pterygodermatites (Neopaucipectines) bovieri (Blanchard, 1886)

Rictularia plagiostoma (Wedl, 1861)!

Rictularia sp.

Riouxgolvania nyctali (Bain \& Chabaud, 1979)

Riouxgolvania rhinolophi (Bain \& Chabaud, 1968)

Riouxgolvania sp.

Seuratum mucronatum (Rudolphi, 1809)

Strongylacantha glycirrhyza van Beneden, 1873

Strongylus tipula P.J. Van Beneden, $1873=$

Molinostrongylus tipula (Beneden, 1873)

Trichosomum speciosum van Beneden, 1873

Uncinaria glycirrhiza $($ van Beneden, 1873$)=$

Strongylacantha glycirrhyza van Beneden, 1873
Myotis myotis (Borkhausen, 1797) ! [233]

Myotis emarginatus (Geoffroy, 1806) [86]

Myotis blythii (Tomes, 1857) and M. blythii oxygnathus Monticelli, 1885 [21, 167]

Miniopterus schreibersii (Natterer in Kuhl, 1817) [21, 167]

Rhinolophus euryale Blasius, 1853 [20, 167]

Myotis blythii (Tomes, 1857) and M. blythii oxygnathus Monticelli, 1885 [86]

Plecotus auritus (Linné, 1758) [54, 86, 194]

Plecotus sp. [97]

Miniopterus schreibersii (Natterer in Kuhl, 1817) [99, 167, 200]

Rhinolophus ferrumequinum (Schreber, 1774) [68, 167, 200, 243]

3

Miniopterus schreibersii (Natterer in Kuhl, 1817) [200]

Rhinolophus ferrumequinum (Schreber, 1774) [200]

Rhinolophus hipposideros (Bechstein, 1800) [200]
Latreille contains an observation of Carios on "la ChauveSouris noctule" (=Nyctalus noctula or Nyctalus sp.). This taxon is very likely Argas (Carios) vespertilionis (Latreille, 1802). Descriptions of some species and subspecies are based on type material from France (e.g. Spinturnix nobleti [81], S. bechsteini [82], Pteracarus pipistrellius maximis [251], Myobia poppei (= Acanthophthirius (Acanthophthirius) poppei) [249] and Spinturnix andegavinus [76]). The majority of published data on parasites of chiropteran populations in France deal with Arachnida and similar findings were noted by Lanza [167], Krištofík \& Danko [165] and Frank et al. [121] in Italy, Slovakia and other European countries.

\subsection{Subphylum Hexapoda Latreille, 1825}

\subsubsection{Suborder Anoplura Leach, 1815} (order Phthiraptera Haeckel, 1896)

Only one species has been reported as a bat parasite in France, i.e. Polyplax serrata. The scientist Paul Rémy (18941962) published in 1948 the only record; this was from
R. hipposideros (Borkhausen, 1797) in north-eastern France at Trémont-sur-Saulx (Meuse area [177, 216]). The mentioned locality in the original paper, "Frémont-sur-Saulx" [216], contains a typographical error. These field data, dated 19251926, and published in the journal La Feuille des Naturalistes, are surprising and dubious [Beaucournu, in litt.]. In fact, this species is more likely to be an ectoparasite of mammals of the Rodentia order (e.g. Apodemus, Clethrionomys and Mus genera) and Eulipotyphla (Crocidura leucodon) [98, 234]. It should be noted, however, that Polyplax sp. was also reported on Rhinolophus mehelyi by Gadžiev et al. in 1990 in Eastern Europe. These are the only data on Polyplax sp. in Lanza's analysis [167] and hence they may be unreliable. Rémy's observation is not mentioned in the works of Durden \& Musser [98], Ferris [119] and Hopkins [140].

\subsubsection{Order Diptera Linnaeus, 1758}

According to Szentiványi et al., 17 species of bat flies are currently known in Europe [237]. Thirteen species of bat flies 


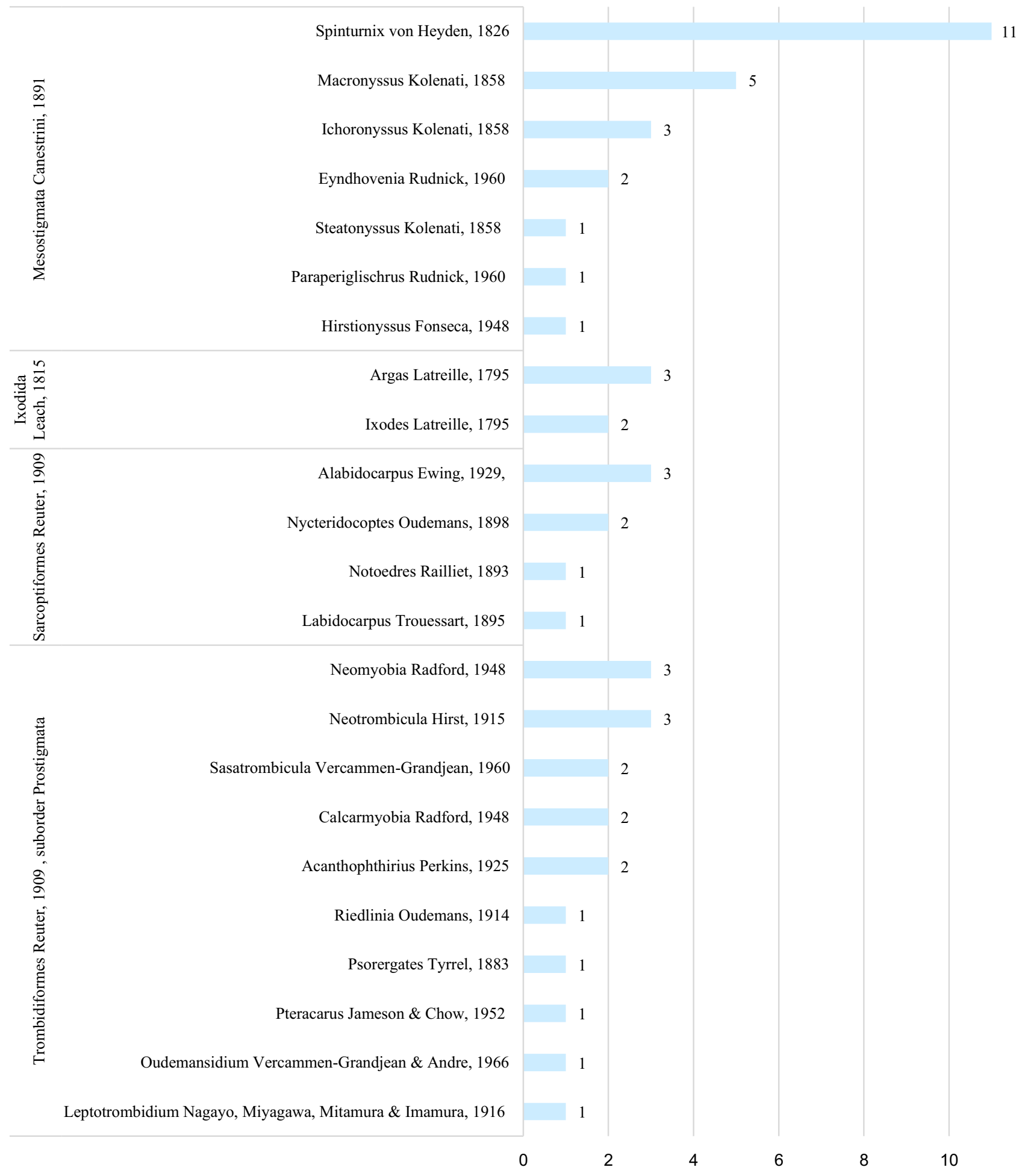

Figure 3. Number of recognised species of Acari $(n=53)$, per host order $(n=4)$ and genus $(n=23)$. Invalid species $(n=22$ Acari) recorded in the literature $(n=237$ papers) and species only noted as absent $(n=3$ Acari) are not included here.

have been reported from France and two more records without identification to species level have been found. These are Basilia (Basilia) italica, B. (Basilia) mediterranea, B. (Basilia) nana, 1954, B. (Basilia) nattereri, Brachytarsina flavipennis, Nycteribia (Achrocholidia) vexata, N. (Nycteribia) kolenatii, N. (Nycteribia) latreillii, N. (Nycteribia) pedicularia,
N. (Nycteribia) schmidlii, Penicillidia (Neopenicillidia) conspicua, P. (Penicillidia) dufourii, Phthiridium biarticulatum, Nycteribia sp. and Nycteribia kolenatii/latreillii/pedicularia $[1,4,12,22-30,34,35,38-41,43,45,47,53,73,74,86$, $91,93,115-117,126,135,146-152,154,156,167,170$, $172,173,175,177,179,186,187,200,203,204,206,214$, 


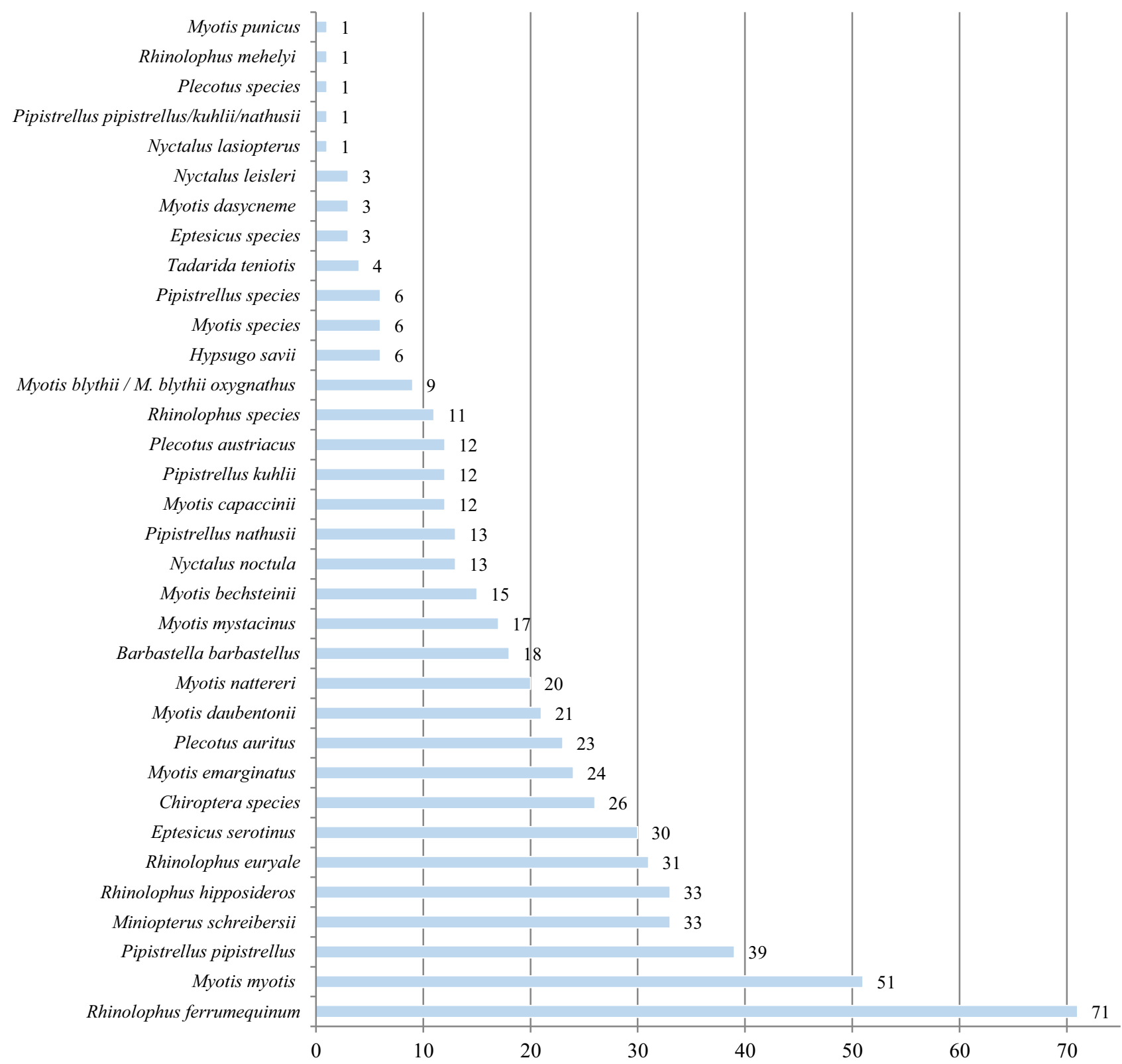

Figure 4. Histogram showing the number of studies $(n=237)$ per host taxon $(n=34$; species: 27 ; complex: 1 ; genera: 6$)$ during the period 1762-2018.

215, 237, 240-242, 247, 248, 260]. Three invalid taxa reported from France were found in the analysed papers: Nycteribia eparticulata, N. vespertilionis Meig., and N. vespertilionis Latreille. Penicillidia (Penicillidia) monoceros is noted as absent in western France [28]. Another species, B. (Basilia) daganiae could be distributed in France [28, 34]. As far as hosts of the bat flies (Diptera: Nycteribiidae and Streblidae) are concerned, E. serotinus, H. savii, M. schreibersii, M. bechsteinii, M. blythii, M. capaccinii, M. daubentonii, M. emarginatus, M. myotis, M. mystacinus, $M$. nattereri, $M$. species, $P$. pipistrellus, Pipistrellus sp., P. auritus, $R$. euryale, R. ferrumequinum, $R$. hipposideros, and $R$. mehelyi have been recorded in the literature. Jean-Frédéric Hermann's record of Phthiridium vespertilionis, dated 1804, is the oldest French record of a dipteran as a bat parasite [135]. The bibliographical survey of the published data ( $n=111$ papers) written by Szentiványi et al. has shown that ten bat fly species are known to be associated with bats in Albania, Romania, and Italy [237]. Europe's most species-rich communities have been reported in Spain (11 species), Switzerland (11 species), Hungary (11 species) and France (13 species). As such, France has the most diverse community reported in the literature.

\subsubsection{Order Hemiptera Linnaeus, 1758}

The literature provides well-documented cases for Cimex dissimilis, C. lectularius, and C. pipistrelli on M. myotis, $M$. emarginatus, $R$. euryale, and $R$. ferrumequinum [28, 167, $177,207,236,253,254]$. In addition to these species, records 


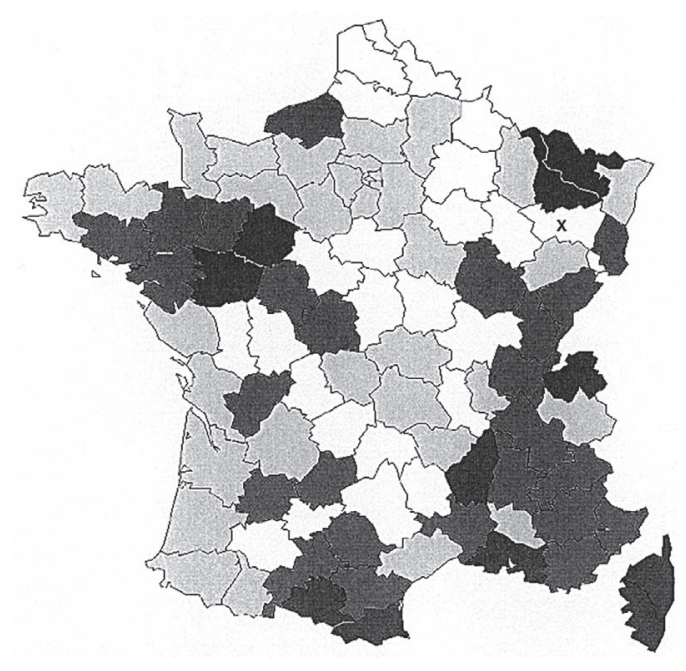

\begin{tabular}{|l|l|l|}
\hline $\begin{array}{l}>10 \quad \text { papers } \\
(\max =24)\end{array}$ & & $\begin{array}{l}\text { Department not } \\
\text { mentioned in } \\
\text { literature }\end{array}$ \\
\hline $5-10$ papers & $\mathbf{X}$ & $\begin{array}{l}\text { Non-studied area } \\
\text { between 1762 and } \\
2018\end{array}$ \\
\hline$<5$ papers & \multicolumn{2}{|l}{} \\
\hline
\end{tabular}

Figure 5. Study area and the number of publications that include data on parasites of bats in each French administrative region (department).

reported from France without identification to species level have been found (Cimex sp. on M. myotis, M. emarginatus, $R$. euryale, and $R$. ferrumequinum) [28, 177]. The first published data in the analysed literature are dated 1961 [28] when Beaucournu published his observations on Cimex sp. in the department of Maine-et-Loire. True bugs (Hemiptera: Cimicidae) using bats as hosts have not been well studied in France. Studies on bat guano deposits could provide data on the species distribution. For instance, this method provided new records of Cimex sp. and Cimex dissimilis (Horváth, 1910) in a roosting colony of M. myotis in June 2017 and June 2018 in north-eastern France [177] (about $C$. dissimilis in roosting colony of M. myotis, see also [121] and [224]).

\subsubsection{Order Siphonaptera Latreille, 1825}

Twelve species are known to be associated with bats in France. They belong to the family Ischnopsyllidae Wahlgren, 1907. The species are Araeopsylla gestroi, Ischnopsyllus (Hexactenopsylla) hexactenus, I. (Ischnopsyllus) elongatus, I. (Ischnopsyllus) intermedius, I. (Ischnopsyllus) octactenus, I. (Ischnopsyllus) simplex, I. (Ischnopsyllus) variabilis, Nycteridopsylla ancyluris ancyluris, $N$. eusarca, N. longiceps, $N$. pentactena, and Rhinolophopsylla unipectinata unipectinata. According to Beaucournu and Launay [37], the published records of Nycteridopsylla dictena are dubious [146, 227]. Bat fleas have been observed on 20 hosts in France [3, 14, 23, 29, 33, 36-38, 42-44, 46, 85, 86, 90, 95, 141, 142, 152, 156, 157, 167, 177, 200, 204, 219, 227, 228, 231, 242, 257]. These hosts are E. serotinus, M. schreibersii, M. blythii,
M. capaccinii, M. daubentonii, M. emarginatus, M. myotis, M. mystacinus, M. nattereri, Myotis sp., N. leisleri, $N$. noctula, P. kuhlii, P. nathusii, P. pipistrellus, P. auritus, P. austriacus, $R$. euryale, $R$. ferrumequinum, $R$. hipposideros, Rhinolophus sp., and Tadarida teniotis. Studies on bat fleas have a long history in France and the book of Walckenaer on insects, entitled Faune parisienne, insectes ou Histoire abrégée des insectes des environs de Paris [257], may be among the earliest such works. According to the Inventaire National du Patrimoine Naturel, 91 autochthonous (indigenous) species of the Siphonaptera order have been reported in France. Species of the Ischnopsyllidae found in France represent almost $13 \%$ of fleas in the country [153].

\section{Phylum Nematoda Diesing, 1861}

In France, the helminth fauna of bats is varied, with more than 30 species. Nematodes recorded in bats in France are divided into three orders: Muspiceida Bain \& Chabaud, 1959, Rhabditida Chitwood, 1933, and Strongylida Molin, 1861; and six families: Onchocercidae Leiper, 1911 (three species), Molineidae Skrjabin \& Schulz, 1937 (four species), Rictulariidae Railliet, 1916 (one species), Muspiceidae Bain \& Chabaud, 1959 (two species), Seuratidae Hall, 1916 (one species), and Strongylacanthidae (Yorke \& Maplestone, 1926, subfamily) Chabaud, 1960 (one species). Thirteen species and two nematodes identified to genus-level have been reported: Litomosa dogieli, L. filaria, L. ottavianii, Molinostrongylus alatus, M. ornatus, M. panousei, M. tipula, Pterygodermatites (Neopaucipectines) bovieri, Riouxgolvania nyctali, $R$. rhinolophi, Seuratum mucronatum, Strongylacantha glycirrhyza, Rictularia sp., Riouxgolvania sp., and Trichosomum speciosum. These parasites were documented in the following bat species: $M$. schreibersii, M. blythii, M. emarginatus, M. myotis, Plecotus sp., Rhinolophus sp. and three species of the genus Rhinolophus ( $R$. euryale, $R$. ferrumequinum, and R. hipposideros) [19-21, 54, 56, 57, 68, 75, 86, 97, 99, 167, $177,194,200,211,233,235,243,245]$. The original description of the rare nematode $P$. (Neopaucipectines) bovieri is based on material from $M$. myotis in France. To my knowledge, it is the first published observation in the country (dated September 1885) [56, 66, 230]. The original descriptions of Riouxgolvania nyctali and $R$. rhinolophi are based on material from $M$. blythii and $R$. euryale in the Netherlands and the French departments of Ariège and Pyrénées-Orientales [20, 21]. A re-description of Seuratum mucronatum was based on material from Plecotus auritus (dated 1950) in the French department of Indre-etLoire [54]. As a comparison, according to Horvat et al. [144, 145], two Nematode species (associated with M. myotis and $R$. ferrumequinum) are known in Serbia to be associated with bats, whilst in Croatia, these authors noted three species.

\section{Phylum Platyhelminthes Minot, 1876}

\subsection{Class Trematoda}

Records of 15 recognised species of trematodes from bats have been found from over 17 published papers. They belong to the order Plagiorchiida La Rue, 1957 and are divided into 
three families: Lecithodendriidae Lühe, 1901 (13 species), Mesotretidae Poche, 1926 (one species), and Plagiorchiidae Lühe, 1901 (one species). These species are Allassogonoporus amphoraeformis, Lecithodendrium granulosum, L. linstowi, L. moedlingeri, Mesotretes peregrinus, Parabascus duboisi, P. lepidotus, P. semisquamosus, Plagiorchis vespertilionis, Prosthodendrium (Prosthodendrium) carolinum, P. (Prosthodendrium) hurkovaae, P. (Prosthodendrium) chilostomum, $P$. (Prosthodendrium) longiforme, $P$. parvouterus, and Pycnoporus heteroporus [1, 57, 60, 61, 68, 71, 86-89, 97, 163, 167, 177, 188, 200, 202, 243]. Two trematodes identified to genus-level have been reported (Lecithodendrium sp. and Prosthodendrium sp.) [86]. These parasites were documented in the following bat species: Eptesicus serotinus [68, 88, 167], Miniopterus schreibersii [68, 167, 188, 200, 235], Myotis daubentonii [89, 167], M. capaccinii [68, 167], M. emarginatus [68, 86, 167], M. myotis [57, 61, 68, 167], Pipistrellus kuhlii [86], P. pipistrellus [57, 60, 61, 68, 86, 97, 163, 167, 235], Plecotus auritus [86, 167], P. austriacus [68, 167], Rhinolophus euryale [68, 167], R. ferrumequinum [68, 86-88, $167,188,200,243]$, and $R$. hipposideros [68, 86, 167, 188, 200, 235].

As a comparison, in the United Kingdom, Lord et al. [181, 182] noted four trematode species and one trematode identified to genus-level in bats. In Serbia, Horvat et al. [144, 145] noted a total of seven trematode taxa associated with bats, and only one species in Croatia. According to the Inventaire National du Patrimoine Naturel, 28 species of the family Lecithodendriidae and 27 species of the family Plagiorchiidae have been reported in France. Species of the Lecithodendriidae found in bats represent almost $46 \%$ of the family [153]. The observations on Mesotretes peregrinus (Braun, 1900), published by Combes \& Clerc, Dubois and Matskási [68, 88, 188], are of particular interest since this is the only species of the family Mesotretidae reported in Europe [125, 153]. As regards Plagiorchis vespertilionis (O.F. Müller, 1784) [68, 86-89, 200, 235], it is worth noting that this species is the only member of the family Plagiorchiidae reported from bats in France. According to Lanza [167], 18 taxa of the Plagiorchiidae family have been reported worldwide. The Histoire naturelle des helminthes ou vers intestinaux (1845) by Félix Dujardin (1801-1860) appears to be the earliest French source mentioning bat-associated trematodes [93]. The first documented reports from France, P. (Prosthodendrium) chilostomum and P. heteroporus, were published in the Notices helminthologiques (deuxième série) by Raphaël Blanchard (1857-1919) [57].

\subsection{Class Cestoda}

Three recognised and one innominate species of cestodes have been reported in bats in France, which makes it the richest community reported in Europe. These species are Milina grisea, Vampirolepis acuta, and V. balsacii [68, 86, 158, 159, 167, 177, 180, 196, 200, 243, 255, 262]. They belong to one family: Hymenolepididae Ariola, 1899 (Cyclophyllidea van Beneden in Braun, 1900 order). In addition to these species, one cestode identified to genus-level has been reported (Vampirolepis sp.) [86]. The description of H. balsacii is based on material from Myotis bechsteinii and Eptesicus serotinus from north-eastern France collected by the naturalist Henri Heim de Balsac (1899-1979) at a place called Buré d'Orval in Allondrelle-la-Malmaison [158, 159]. Some authors have noted that the observation was made at "Buré" ([158] see also [142]). Joyeux \& Baer's paper on cestodes, entitled Sur quelques Cestodes de France, is the first work on the bat cestodes in France (published in 1934 in the journal Archives du Muséum national d'Histoire naturelle). Bat cestodes are not well studied, especially in France, where the most recent data from field research is almost 50 years old [68, 86, 158, $159,167,177,180,196,200,243,255,262]$. France has the most diverse community reported in the literature. According to Frank et al. [121], three species are known to be associated with bats in Poland and Hungary. These authors also reported one species in Germany and two species in Austria. It is worth noting that, according to the Inventaire National du Patrimoine Naturel, 75 species of the family Hymenolepididae have been reported in France and species of the family found in bats represent $4 \%$ of the family [153].

\section{Hosts and geographical distribution of bat-parasite associations}

Over a 256-year period, the 113 recognised taxa of bat parasites from France were collected from 27 bats species and six other bats that were not identified to species-level (five genera and the Pipistrellus species complex) (Figs. 2 and 4). The taxa are B. barbastellus, E. serotinus, H. savii, M. schreibersii, M. bechsteinii, M. blythii, M. capaccinii, M. dasycneme, M. daubentonii, M. emarginatus, M. myotis, M. mystacinus, M. nattereri, M. punicus, N. lasiopterus, N. leisleri, N. noctula, P. kuhlii, P. nathusii, P. pipistrellus, P. auritus, P. austriacus, $R$. euryale, $R$. ferrumequinum, $R$. hipposideros, $R$. mehelyi, T. teniotis, Eptesicus sp., Myotis sp., Pipistrellus sp., Plecotus sp., Rhinolophus sp., and the species complex Pipistrellus pipistrellus/kuhlii/nathusii. These species represent almost $79 \%$ of the bat fauna of France (including Corsica). The most commonly reported hosts, which are mentioned in more than 29 papers, are E. serotinus, $R$. euryale, $R$. hipposideros, M. schreibersii, P. pipistrellus, M. myotis, and R. ferrumequinum. However, the most cited species is the Greater horseshoe bat ( $R$. ferrumequinum); 30\% of the analysed publications deal with this species. Some bat species have no records of associated metazoan parasites in the analysed publications ( $n=237$ ), because the ecology of the host is poorly studied (E. nilssonii, Vespertilio murinus (Particoloured Bat), M. alcathoe, M. escalerai and P. macrobullaris). In addition to this, since $P$. pygmaeus was identified in the 1990 's, we cannot rule out that some of the records of Pipistrellus sp. or $P$. pipistrellus may refer to $P$. pygmaeus $[41,45,53,58,142]$.

Published field data originated from 72 French departments (Fig. 5). One of them is mentioned as a non-prospected area (department of Vosges). Indeed, there is no publication about bat parasites in this department. Associations with specified geographical locations were most commonly from Ardèche (11 papers), Ariège (13 papers), Bouches-du-Rhône (15 papers), Haute-Savoie (12 papers), Maine-et-Loire (19 papers), Moselle (11 papers), Meurthe-et-Moselle (23 papers), PyrénéesOrientales (24 papers), Sarthe (12 papers), Haute-Corse, and 
Corse-du-Sud (23 papers). Importantly, these distribution patterns are influenced more by biased sampling efforts than by actual geographical and ecological patterns. This distribution map only helps to point to well-studied areas. The relative prominence of the departments Ardèche, Ariège, PyrénéesOrientales, Haute-Corse and Corse-du-Sud compared to other departments is most likely a result of the attention given to karstic areas by European biospeleology (for instance [14, 23-25, $48-53,69,70,115,118,120,126,131-133,136,137,151$, $152,154,178,186,214-216,246])$. This is closely linked to the success and prevalence of $M$. schreibersii, $R$. euryale, and $R$. ferrumequinum compared to other bat species. This prevalence could be the result of their ability to roost in the summer in limestone areas and underground sites. The focus on E. serotinus, $R$. ferrumequinum, $R$. hipposideros, $P$. pipistrellus, and M. myotis is the result of their ability to exploit anthropogenic environments (i.e. farmland, urban areas). As a consequence of this, these species have more contact with human populations (about $P$. pipistrellus, see [182]) and were the first to be studied in France (for the 1762-1844 period see [11, 13, 18, 90-92, 94-97, 124, 183, 220, 258, 260]).

\section{Conflict of interest}

The author declares no conflict of interest.

Acknowledgements. One of the most pleasant parts of finishing a paper is to thank those who have contributed to the making of it. My thanks first of all to Professor Jean-Lou Justine, Editor-in-chief of Parasite. I have every reason to be grateful for the help and advice of the highly esteemed naturalist Professor Jean-Claude Beaucournu. I would like to express my warmest thanks to the Parasite journal reviewers for proofreading the manuscript and for their valuable comments and suggestions, and to Gilles Le Guillou. My thanks to my father François Léger and Jean-Baptiste Schweyer (Agence Française pour la Biodiversité), who provided me opportunities to deal with biased sampling efforts issues. I extend my warm thanks to Valeria Dragoni and to my friends and colleagues Clément Chardon and Hugo Weissbart (Imperial College London, Department of Bioengineering). I want to thank especially two librarians and colleagues: Lucien Gournay, a fine librarian at the Bibliothèque Mammifères et Oiseaux in Paris (MNHN, service Recherche, enseignement, expertise), who read and commented on the completed manuscript; Ann Qualtrough (Bibliothèque centrale du MNHN, service Collecte, traitement et flux), who took the time to read the manuscript and make numerous valuable suggestions. Of course, it needs to be added that responsibility for any remaining errors is my own.

\section{References}

1. Aellen V. 1955. Étude d'une collection de Nycteribiidae et de Streblidae (Diptera pupipara) de la région paléarctique occidentale, particulièrement de la Suisse. Bulletin de la Société Neuchâteloise des Sciences Naturelles, 78, 81-104.

2. Aellen V. 1960. Notes sur les puces des chauves-souris, principalement de la Suisse (Siphonaptera : Ischnopsyllidae). Bulletin de la Société Neuchâteloise des Sciences Naturelles, 83, 41-61.

3. Aellen V. 1962. Le baguement des chauves-souris au Col de Bretolet (Valais). Archives de Sciences, 14, 365-392.
4. Aellen V. 1963. Les nyctéribiidés de la Suisse, diptères parasites des chauves-souris. Bulletin de la Société Neuchâteloise des Sciences Naturelles, 86, 143-154.

5. Anciaux de Faveaux M. 1976. Catalogue des Acariens parasites et commensaux des Chiroptères. Septième partie : mise à jour des troisième et quatrième parties. Addendum. Documents de travail de l'Institut Royal des Sciences Naturelles de Belgique, 7, 546-638.

6. Anciaux de Faveaux M. 1987. Catalogue des Acariens parasites et commensaux des Chiroptères. Dixième partie: Index récapitulatif de tout le catalogue. Documents de travail de l'Institut Royal des Sciences Naturelles de Belgique, 7, 1028-1128.

7. Anciaux de Faveaux M. 1987. Catalogue des Acariens parasites et commensaux des Chiroptères. Neuvième partie: mise à jour des troisième et quatrième parties. Documents de travail de l'Institut Royal des Sciences Naturelles de Belgique, 7, 372-1027.

8. André M. 1930. Communications. Une espèce de Thrombicula [Acarien] non encore signalée en France. Bulletin de la Société Entomologique de France, 15, 237-239.

9. André M. 1932. Nouvelles observations sur les Thrombicula (Acariens). Archivio Zoologico Italiano, 16(3-4), 1355-1362.

10. André M. 1933. Une espèce de Thrombicula autumnalis Shaw ou T. russica Oud. ? [Acariens]. Bulletin de la Société Entomologique de France, 38, 154-156.

11. Anonyme. 1845. Acari, Reports on the Progress of Zoology and Botany. 1841-1842. The Ray Society: Edinburgh. 514 p. (pp. 267-269).

12. Anonyme. 1938. Procès-verbaux des séances d'octobre 1938. Section entomologique. Séance du 19 octobre. Bulletin Mensuel de la Société Linnéenne de Lyon, 7(9), 241-244.

13. Anonyme. 1842. Séance du 3 août 1842 . Annales de la Société Entomologique de France, 11, XXXIV-XLIX.

14. Ariagno D. 1973. Observations sur une colonie de petits et de grands murins (Myotis oxygnatus et Myotis myotis). Annales de Spéléologie, 28(1), 125-130.

15. Arthur DR. 1956. The Ixodes ticks of Chiroptera (Ixodoidea, Ixodidae). Journal of Parasitology, 4(1), 180-196.

16. Arthur L, Lemaire M. 2015. Les Chauves-souris de France, Belgique, Luxembourg et Suisse. Biotope and Muséum National d'Histoire Naturelle publishers: Mèze and Paris. 544 p.

17. Artois M, Hamon B, Léger F, Schwaab F. 1986. Contribution à l'étude du Petit rhinolophe en Lorraine Cas particulier d'une colonie de mise-bas dans le Toulois. Rapport final, Groupe d'Étude des Mammifères de Lorraine (GEML) and Parc Naturel Régional de Lorraine (PNRL) Report, $64 \mathrm{p}$.

18. Audouin JV. 1832. Lettres pour servir de matériaux à l'histoire des Insectes. Première Lettre, contenant des Recherches sur quelques Araignées parasites des genres Ptéropte, Caris, Argas et Ixode, adressée à M. Léon Dufour, correspondant de l'Institut. Annales des Sciences Naturelles, 25, 401-425.

19. Bain O. 1967. Diversité et étroite spécificité parasitaire des filaires de chauves-souris, confondues sous le nom de Litomosa filaria (van Beneden, 1872). Bulletin du Muséum National d'Histoire Naturelle, 2nd serie, 38, 928-939.

20. Bain O, Chabaud A-G. 1968. Description de Riouxgolvania rhinolophi n. g., n. sp., Nématode parasite de Rhinolophe, montrant les affinités entre Muspiceoidea et Mermithoidea. Annales de Parasitologie Humaine et Comparée, 43(1), 45-50.

21. Bain O, Chabaud A-G. 1979. Sur les Muspiceidae (NematodaDorylaimina). Annales de Parasitologie Humaine et Comparée, 54(2), 207-225.

22. Balazuc J. 1971. Notes sur les Laboulbéniales. III : Rectifications, synonymies et mises au point (suite). Bulletin Mensuel de la Société Linnéenne de Lyon, 40(7), 211-216. 
23. Balazuc J, Dresco E, Henrot H, Nègre J. 1951. Biologie des carrières souterraines de la région parisienne. Vie et Milieu, 2, 301-334.

24. Balazuc J, de Miré P, Sigwalt J. 1954. Sixième, septième et huitième campagnes biospéologiques dans le Vivarais (août 1951, mai 1952, mai 1953). Bulletin Mensuel de la Société Linnéenne de Lyon, 23(5), 138-143.

25. Balazuc J, de Miré P, Sigwalt J, Théodoridès J. 1951. Trois campagnes biospéléogiques dans le Bas-Vivarais (Avril 1949Décembre 1949, Juin-Juillet-Août 1950). Bulletin Mensuel de la Société Linnéenne de Lyon, 20(8), 187-192.

26. Balcells E. 1955. Datos para el estudio de la fauna pupípara de los quirópteros en España. Revista de Ciencias, 5(1), 283-308.

27. Beaucournu J-C, Beaucournu-Saguez F, Guiguen C. 1985. Nouvelles données sur les Diptères pupipares (Hippoboscidae et Streblidae) de la sous-région méditerranéenne occidentale. Annales de Parasitologie Humaine et Comparée, 85(3), 311-327.

28. Beaucournu J-C. 1961. Ectoparasites des Chiroptères de l'Ouest de la France $1^{\text {re }}$ partie Ixodoïdés - Cimicides et Nyctéribiidés. Bulletin de la Société Scientifique de Bretagne, 26, 315-338.

29. Beaucournu J-C. 1962. Ectoparasites des Chiroptères de l'Ouest de la France $2^{\mathrm{e}}$ partie Siphonaptères - Hôtes et biotopes. Bulletin de la Société Scientifique de Bretagne, 26, 315-338.

30. Beaucournu J-C. 1962. Nouvelles captures de Nycteribiidae (Diptera, Pupipara) en France. Annales de Parasitologie Humaine et Comparée, 37(3), 366-373.

31. Beaucournu J-C. 1966. Sur quelques Ixodoidea (Acarina) paléarctiques inféodés aux micro-Chiroptères. Annales de Parasitologie Humaine et Comparée, 41(5), 495-502.

32. Beaucournu J-C. 1967. Contribution à la connaissance de la biologie d'Ixodes (Eschatocephalus) vespertilionis Koch 1844 et d'Ixodes (Pomerantzevella) simplex Neumann 1906 (Acarina, Ixodoidea), parasites des Chiroptères. Annales de Spéléologie, 22(3), 543-580.

33. Beaucournu J-C. 1968. Catalogue provisoire des Siphonaptères de la faune française. Annales de la Société Entomologique de France, nouvelle série, 4(3), 615-635.

34. Beaucournu J-C. 1972. Seconde capture en France de Basilia italica Theodor, 1954, (Diptera, Nycteribiidae). Présence en Anjou. Bulletin de la Société Scientifique de Bretagne, 47, 119122.

35. Beaucournu J-C. 1985. Insectes parasites et mammifères ou de l'entente cordiale à la guerre totale. Arvicola, 2(2), 71-75.

36. Beaucournu J-C, Gosalbez J. 1975. Contribution à l'étude des Siphonaptères de Catalogne française et espagnole. Vie et Milieu, 35(1), série C, 69-86.

37. Beaucournu J-C, Launay H. 1990. Les puces de France et du Bassin méditerranéen occidental. Collection Faune de France, number 76. Fédération Française des Sociétés des Sciences Naturelles : Paris. p. 550.

38. Beaucournu J-C, Matile L. 1958. Contribution à l'inventaire faunistique des cavités souterraines de l'Ouest de la France. Bulletin de la Société des Sciences Naturelles de l'Ouest de la France, 54, 5-16.

39. Beaucournu J-C, Matile L. 1963. Contribution à l'inventaire faunistique des cavités souterraines de l'Ouest de la France. Annales de Spéléologie, 18(3), 343-357.

40. Beaucournu J-C, Noblet J-F. 1985. Une Nyctéribie (Diptera, Pupipara) nouvelle pour la faune française: présence de Basilia mediterranea Hůrka, 1970 en Corse. Annales de Parasitologie Humaine et Comparée, 60(5), 635-638.

41. Beaucournu J-C, Noblet J-F. 1994. Présence en France continentale de Basilia mediterranea Hůrka, 1970 (Diptera, Nycteribiidae). Bulletin de la Société Entomologique de France, 99(4), 397-400.
42. Beaucournu J-C, Noblet J-F. 1995. Confirmation de la présence en France de la puce Ischnopsyllus (I.) elongatus (Curtis, 1932) (Siphonaptera, Ischnopsyllidae). Bulletin de la Société Entomologique de France, 10, 413-414.

43. Beaucournu J-C, Noblet J-F. 1996. Les diptères pupipares parasites de chauves-souris dans les Alpes et les Préalpes françaises (Diptera, Streblidae et Nycteribiidae). Bulletin de la Société Entomologique de France, 101(3), 235-240.

44. Beaucournu J-C, Noblet J-F. 1998. Les puces de chauves-souris dans les Alpes et les Pré-Alpes françaises (Insecta Siphonaptera - Ischnopsyllidae). Le Rhinolophe, 13, 29-34.

45. Beaucournu J-C, Noblet J-F, Gilot B, Degeilh B. 1999. Les Tiques de Chauves-souris dans les Alpes et les Préalpes françaises (Acarina, Ixodidae et Argasidae). Bulletin de la Société Entomologique de France, 104(3), 299-306.

46. Beaucournu J-C, Rault B. 1962. Contribution à l'étude des Siphonaptères de Mammifères dans la moitié orientale des Pyrénées. Vie et Milieu, 13, 571-597.

47. Beaucournu J-C, Rault B. 1968. Note sur la spécificité, la localisation et la chorologie des Anoploures et de quelques autres Insectes ectoparasites de mammifères en France. Annales de Parasitologie Humaine et Comparée, 43(3), 381-386.

48. Beron P. 1965. Contribution à l'étude des Acariens parasites des Chiroptères en Hongrie (Spinturnicidae, Trombiculidae, Myobiidae et Acaridae). Vertebrata Hungarica, 7(1-2), 141-152.

49. Beron P. 1970. Sur quelques Acariens (Myobiidae, Psorergatidae, Spinturnicidae, Sarcoptidae et Listrophoroidea) de Bulgarie et de l'île de Crète. Bulletin de l'Institut de Zoologie et Musée de Sofia, 32, 143-149.

50. Beron P. 1971. Sur quelques acariens parasites des Mammifères et des Reptiles de France. Bulletin de la Société d'Histoire Naturelle de Toulouse, 107(1-2), 96-102.

51. Beron P. 1972. Aperçu sur la faune cavernicole de la Corse. Collection Série documents. Publications du Laboratoire souterrain du Centre National de la Recherche Scientifique du Moulis (Ariège - France). Number 3, $56 \mathrm{p}$.

52. Beron P. 1973. Catalogue des acariens parasites et commensaux des mammifères en Bulgarie. Bulletin de l'Institut de Zoologie et Musée de Sofia, 37, 167-199.

53. Bezzi M. 1903. Alcune notizie sui ditteri cavernicoli. Rivista Italiana di Speleologia, 1(2), 3-16.

54. Biocca E, Chabaud A-G. 1951. Redescription de Seuratum mucronatum (Rud. 1809) (Nematoda - Cucullanidae). Annales de Parasitologie Humaine et Comparée, 26(1-2), 86-92.

55. Blackwell M. 1980. Incidence, host specificity, distribution, and morphological variation in Arthrorhynchus nycteribiae and A. eucampsipodae (Laboulbeniomycetes). Mycologia, 72(1), 143-158.

56. Blanchard R. 1886. Notices helminthologiques (Première série). Bulletin de la Société Zoologique de France, 11, 294-304.

57. Blanchard R. 1891. Notices helminthologiques (deuxième série). Mémoires de la Société Zoologique de France, 4, 420-489.

58. Bonnet C. 1908. Aperçu sur l'anatomie et la classification des Ixodidés. Faune française des Ixodidés. Archives de Parasitologie, 12, 224-267.

59. Bonnet C. 1908. Eschatocephalus flavipes (Koch), nouvel Ixodidé pour la faune française. Archives de Parasitologie, 12, 325-327.

60. Botella P, Sánchez L, Esteban J-G. 1993. Helminthfauna of bats in Spain. III. Parasites of Pipistrellus pipistrellus (Schreber, 1774) (Chiroptera: Vespertilionidae). Research and Reviews in Parasitology, 53(1-2), 63-70.

61. Braun M. 1900. Trematoden der Chiroptera. Annalen des K.K. Naturhistorischen Hofmuseums, 15(3-4), 217-236 + one plate. 
62. Brumpt É. 1913. Précis de parasitologie. Masson et Cie : Paris. $1011 \mathrm{p}$.

63. Bruyndonckx N, Dubey S, Ruedi M, Christe P. 2009. Molecular cophylogenetic relationships between European bats and their ectoparasites mites (Acari, Spinturnicidae). Molecular Phylogenetics and Evolution, 51, 227-237.

64. Bruyndonckx N, Biollaz F, Dubey S, Goudet J, Christe P. 2010. Mites as biological tags of their hosts. Molecular Ecology, 19, 2770-2778.

65. Buitendijk AM. 1945. Voorloopige catalogus van de acari in de collectie-Oudemans. Zoologische Mededelingen, 24, 281-391.

66. Caspeta-Mandujano JM, Jiménez FA, Peralta-Rodríguez JL, Guerrero JA. 2013. Pterygodermatites (Pterygodermatites) mexicana n. sp. (Nematoda: Rictulariidae) a parasite of Balantiopteryx plicata (Chiroptera) in Mexico. Parasite, 20, 47-54.

67. Colas-Belcour J. 1933. Contribution à l'étude de la biologie de l'Argas vespertilionis Latreille. Bulletin de la Société de Pathologie Exotique, 26, 937-940.

68. Combes C, Clerc B. 1970. Recherches éco-parasitologiques sur l'helminthofaune des Chiroptères dans l'est des Pyrénées. Annales de Parasitologie Humaine et Comparée, 45(5), 537-561.

69. Cooreman J. 1954. Note sur quelques Acariens de la faune cavernicole. Bulletin de l'Institut Royal des Sciences Naturelles de Belgique, 30(34), 1-19.

70. Cooreman J. 1959. Note sur quelques Acariens de la faune cavernicole ( $2^{\text {ème }}$ série). Bulletin de l'Institut Royal des Sciences Naturelles de Belgique, 35(34), 1-40.

71. Cosson E, Albalat F. 2007. Résultats de l'inventaire des chiroptères des massifs Concors-Vautubière-Artigues et Sainte-Victoire. Faune de Provence : bulletin du ConservatoireÉtudes Écosystèmes de Provence-Alpes du Sud, 23, 83-89.

72. Da Fonseca F. 1948. A monograph of genera and species of Macronyssidae Oudemans, 1936 (Synom.: Lyponissidae, Vitzthum, 1931) (Acari). Proceedings of the Zoological Society London, 118, 249-334.

73. Desmarest AG. 1827. Nyctéribie, in Dictionnaire classique d'Histoire naturelle. Tome 12 : NUA-PAM. Ray et Gravier, libraires-éditeurs and Baudouin frères, libraires-éditeurs : Paris. 634 p. (pp. 21-23).

74. Desmarest E. 1867. Séance du 9 janvier 1867. Annales de la Société Entomologique de France, année 1867, I-IV.

75. Desportes C. 1946. Des filaires dans le tube digestif. Annales de Parasitologie Humaine et Comparée, 21(3-4), 138-141.

76. Deunff J. 1977. Observations on Spinturnicidae of occidental paleartic region (Acarina, Mesostigmata) - Specificity, distribution and repartition. Acarologia, 18(4), 602-617.

77. Deunff J. 1982. Observations en microscopie électronique à balayage sur la famille des Spinturnicidae (Acarina, Mesostigmata). I Morphologie générale. Acarologia, 23(2), 103-111.

78. Deunff J, Beaucournu J-C. 1981. Phénologie et variations du dermecos chez quelques espèces de Spinturnicidae (Acarina, Mesostigmata). Annales de Parasitologie Humaine et Comparée, 56(2), 203-224.

79. Deunff J, Keller A, Aellen V. 1986. Découverte en Suisse d'un parasite nouveau, Spinturnix helvetiae $\mathrm{n}$. sp. (Acarina, Mesostigmata, Spinturnicidae), spécifique de Nyctalus leisleri (Chiroptera, Vespertilionidae). Revue Suisse de Zoologie, 93(3-4), 803-812.

80. Deunff J, Keller A, Aellen V. 1997. Redescription of Spinturnix punctata (Sundevall, 1833) (Acari, Mesostigmata, Spinturnicidae), a specific parasite of Barbastella barbastellus (Chiroptera, Vespertilionidae). Revue Suisse de Zoologie, 104(1), 199-206.

81. Deunff J, Volleth M, Keller A, Aellen V. 1990. Description de Spinturnix nobleti n. sp. (Acari, Mesotigmata, Spinturnicidae), parasite spécifique de Pipistrellus (Hypsugo) savii (Chiroptera, Vespertilionidae). Revue Suisse de Zoologie, 97, 477-488.
82. Deunff J, Walter G, Bellido A, Volleth M. 2004. Description of a cryptic species, Spinturnix bechsteinii n. sp. (Acari, Mesostigmata, Spinturnicidae), parasite of Myotis bechsteinii (Kuhl, 1817) (Chiroptera, Vespertilionidae) by using ecoethology of host bats and statistical methods. Journal of Medical Entomology, 41(5), 826-832.

83. Dick CW, Dittmar K. 2014. Parasitic bat flies (Diptera: Streblidae and Nycteribiidae): Host specificity and potential as vectors, in Bats (Chiroptera) as Vectors of Diseases and Parasites. Klimpel S, Mehlhorn H, Editors. Springer: Berlin. 187 p. (pp. 131-155).

84. Dietz C, Helversen O(von), Nill D. 2009. Bats of Britain, Europe and Northwest Africa. A \& C Black: London. 400 p.

85. Didier R, Rode P. 1935. Les Mammifères de France. Collection Archives d'Histoire Naturelle. Société Nationale d'Acclimatation de France : Paris. 398 p. (pp. 111-114).

86. Dollfus RP. 1961. Chapitre premier. Liste des parasites par hôtes. Annales de Parasitologie Humaine et Comparée, 36(3), 174-261.

87. Dubois G. 1955. Les Trématodes de Chiroptères de la collection Villy Aellen. Étude suivie d'une révision du sous-genre Prosthodendrium Dollfus 1937 (Lecithodendriinae Lühe). Revue Suisse de Zoologie, 62, 469-506.

88. Dubois G. 1956. Contribution à l'étude des trématodes des Chiroptères. Revue Suisse de Zoologie, 63, 683-695.

89. Dubois G. 1963. Contribution à l'étude des Trématodes de Chiroptères. Révision du genre Allassogonoporus Olivier 1938 et note additionnelle sur le sous-genre Prosthodendrium Dollfus 1931. Revue Suisse de Zoologie, 70(6), 103-125.

90. Dufour L, Audouin JV. 1832. Extrait d'une lettre de M. Léon Dufour à M. Audouin, sur le Pteroptus vespertilionis. Annales des Sciences Naturelles, 26, 257-260.

91. Dufour L. 1831. Description et figures de la Nyctéribie du Vespertilion et observations sur les stigmates des insectes pupipares. Annales des Sciences Naturelles, 22, 372-384 + one plate.

92. Dufour L. 1832. Description et figure du Pteroptus Vespertilionis, insecte nouveau de la famille des Tiques. Annales des Sciences Naturelles, 26, 98-102 + one plate.

93. Dufour L. 1845. Études anatomiques et physiologiques sur les insectes Diptères de la famille des pupipares. Annales des Sciences Naturelles, série 3, tome 3, 49-95 + 2 plates.

94. Dugès A. 1834. Recherches sur l'ordre des Acariens en géneral et la famille des Trombidiés en particulier. Premier Mémoire. Annales des Sciences Naturelles, Zoologie, série 2, tome 1, 5-46.

95. Dugès A. 1832. Recherches sur les caractères zoologiques du genre Pulex, et sur la multiplicité des insectes qu'il renferme. Annales des Sciences Naturelles, 27, 145-165 + one plate.

96. Dujardin F. 1842. Nouveau manuel complet de l'observateur au microscope. Librairie encyclopédique de Roret: Paris. 44 p. +30 plates.

97. Dujardin F. 1845. Histoire naturelle des helminthes ou vers intestinaux, 2 volumes. Roret : Paris. 654 p. and 15 p.

98. Durden LA, Musser GG. 1994. The Mammalian hosts of the sucking lice (Anoplura) of the world: a host-parasite list. Bulletin of the Society for Vector. Ecology, 19(2), 130-168. [Nota bene : the same work has been published with this bibliographical reference : Durden LA, Musser GG. 1994. The sucking lice (Insecta, Anoplura) of the world: a taxonomic checklist with records of mammalian hosts and geographical distributions. Bulletin of the American Museum of Natural History, 218, 1-90.].

99. Durette-Desset M-C, Chabaud A-G. 1975. Nématodes Trichostrongyloidea parasites de Microchiroptères. Annales de Parasitologie Humaine et Comparée, 50(3), 303-307.

100. Dusbábek F. 1963. Parasitische Fledermausmilben der Tchechoslowakei. III. Fam. Myobiidae (Acarina, Trombidiformes). Časopis Československé Společnosti Entomologické (Acta Societatis Entomologicae Cechosloveniae), 60, 231-251. 
101. Dusbábek F. 1964. Contribution à la connaissance des Acariens (Acarina) parasites des Chiroptères de Bulgarie. Acarologia, 6(1), 5-25.

102. Dusbábek F. 1969. Generic revision of the Myobiid mites (Acarina: Myobiidae) parasitic on bats. Folia Parasitologica, $16,1-17$.

103. Erichson WF. 1843. Bericht über die wissenschaftlichen Leistungen im Gebiete der Etomologie während des Jahres 1842. Nicolai'schen Buchhandlung : Berlin. 140 p. (p. 133).

104. Evans GO, Till WM. 1968. Studies on the British Dermanyssidae (Acari: Mesostigmata). Part II. Classification. Bulletin of the British Museum (Natural History) (1966), 14(6), 107-370.

105. Eyndhoven GL(van). 1941. Über die Frage der Synonymie von Spinturnix euryalis G. Canestrini 1884 und Periglischrus interruptus Kolenati 1856, sowie über einen neuen Fledermausparasiten, Spinturnix oudemansi nov. spec. (Acar. Spint.). Tijdschrift voor Entomologie, 84, 44-67.

106. Fain A. 1959. Les Acariens psoriques parasites des Chauvessouris. Acarologia, 1(3), 324-334.

107. Fain A. 1959. Les Acariens psoriques parasites des Chauvessouris VII. - Nouvelles observations sur le genre Nycteridocoptes Oudemans 1898. Acarologia, 1(3), 335-353.

108. Fain A. 1959. Les Acariens psoriques parasites des Chauvessouris IX. Nouvelles observations sur le genre Psorergates Tyrrell. Bulletin et Annales de la Société Entomologique de Belgique, 95(7-8), 232-248.

109. Fain A. 1959. L'importance générique de la structure des épimères postérieures du mâle dans les familles Sarcoptidae Trouessart et Teinocoptidae Fain (Acarina - Sarcoptiformes). Acarologia, 1(2), 257-260.

110. Fain A. 1971. Les Listrophorides en Afrique au sud du sahara (Acarina : Sarcoptiformes). II. Familles Listrophoridae and Chirodiscidae. Acta Zoologica et Pathologica Antverpiensia, 54, 5-231 and II-IV.

111. Fain A. 1982. Notes sur les Labidocarpines (Acari, Chirodiscidae) parasites des Chiroptères. Bulletin de l'Institut Royal des Sciences Naturelles de Belgique, 54(4), 1-37.

112. Fain A. 1982. Spécificité et évolution parallèle hôtes-parasites chez les Myobiidae (Acari). In Colloque international du CNRS. Deuxième symposium sur la spécificité parasitaire des parasites des vertébrés. 13-17 avril 1981. Mémoires du Muséum National d'Histoire Naturelle, Série A, Zoologie, nouvelle série, $123,77-85$.

113. Fain A, Aellen V. 1961. Les acariens psoriques parasites des chauves-souris. XX. Un cas d'hyperparasitisme par Nycteridocoptes poppei. Nouvelles observations sur l'évolution cyclique de la gale sarcoptique chez les chiroptères. Revue Suisse de Zoologie, 68(31), 305-309.

114. Fain A, Aellen V. 1979. Les Myobiidae (Acarina, Prostigmata) parasites des Chauves-souris de Suisse I. Revue Suisse de Zoologie, 86(1), 203-220.

115. Falcoz L. 1923. Biospeologica XLIX. Pupipara (Diptères) ( $1^{\text {ère }}$ série). Archives de Zoologie expérimentale et générale : Histoire naturelle-Morphologie-Histologie-Évolution des animaux, 61, 521-552.

116. Falcoz L. 1924. Diptères pupipares du Muséum National d'Histoire Naturelle de Paris (Streblidæ et Nycteriibidæ). [Suite.]. Bulletin du Muséum National d'Histoire Naturelle, 30 (4), 309-315.

117. Falcoz L. 1926. Faune de France. 14 : Diptères pupipares. Collection Faune de France, number 14. Paul Lechevalier et fils : Paris. 64 p.

118. Feider Z. 1970. Nouvelles espèces de Trombiculides dans les grottes d'Europe, in Académie de la République Socialiste de Roumanie. Livre du Centenaire Emile Racovitza 1868-1968.
Éditions de l'Académie de la République Socialiste de Roumanie : Bucarest. 699 p. (pp. 333-350).

119. Ferris GF. 1951. The sucking lice. Collection Memoirs of the Pacific Coast Entomological Society. Pacific Coast Entomological Society: San Francisco. 330 p.

120. Florentin R. 1903-1904. La faune des grottes de Sainte Reine. Feuille des Jeunes Naturalistes, 34, 176-179.

121. Frank R, Kuhn T, Werblow A, Liston A, Kochmann J, Klimpel S. 2015. Parasite diversity of European Myotis species with special emphasis on Myotis myotis (Microchiroptera, Vespertilionidae) from a typical nursery roost. Parasite \& Vectors, 8, 101-114.

122. Fuller HS. 1952. The mite larvae of the family Trombiculidae in the Oudemans collection: taxonomy and medical importance. Zoologische verhandelingen. Rijksmuseum van Natuurlijke Historie te Leiden. E.J. Brill : Leiden. 262 p.

123. Geoffroy ÉL. 1762. Histoire abrégée des insectes: qui se trouvent aux environs de Paris: dans laquelle ces animaux sont rangés suivant un ordre méthodique, 2 volumes. Chez Durand : Paris. $523 \mathrm{p}+10$ plates and 690 p. +22 plates.

124. Gervais P. 1841. Note sur quelques espèces de l'ordre des Acariens. Annales des Sciences Naturelles, $2^{\text {nd }}$ serie, 15, 5-10.

125. Gibson DI, Bray RA, Harris EA(Compilers). 2005. HostParasite Database of the Natural History Museum, London. https://www.nhm.ac.uk/research-curation/scientific-resources/ taxonomy-systematics/host-parasites/.

126. Ginet R. 1952. La grotte de la Balme (Isère): topographie et faune. Bulletin Mensuel de la Société Linnéenne de Lyon, 21(1), 4-17.

127. Godron DA. 1862. Zoologie de la Lorraine ou Catalogue des animaux sauvages observés jusqu'ici dans cette ancienne province. Mémoires de l'Académie Stanislas, 1862, 355-643.

128. Groupe Chiroptères de la Ligue de Protection des Oiseaux Rhône-Alpes. 2014. Les chauves-souris de Rhône-Alpes. Ligue de Protection des Oiseaux Rhône-Alpes : Lyon. 480 p.

129. Haelewaters D, Pfliegler WP, Szentiványi T, Földvári M, Sándor AD, Barti L, Camacho JJ, Gort G, Estók P, Hiller T, Dick CW. 2017. Parasites of parasites of bats: Laboulbeniales (Fungi: Ascomycota) on bat flies (Diptera: Nycteribiidae) in central Europe. Parasites \& Vectors, 10(1), 96.

130. Haitlinger R, Piksa K. 2012. First record of Spinturnix bechsteini (Acari: Mesostigmata: Spinturnicidae) from Poland with remarks on the diagnostic value of some characters. Annals of Parasitology, 58(1), 15-18.

131. Hamon B. 1990. Note sur la découverte de la première colonie d'hibernage de Noctules communes (Nyctalus noctula, Schreber, 1774) en Lorraine. Bulletin de la Société d'Histoire Naturelle de la Moselle, 45, 197-207.

132. Hamon B, Morin D. 1986-1987. Les Chauves-souris de la grotte de la Baume-Noire - Frétigney (70). Bulletin de la Société d'Histoire Naturelle du Doubs, 83, 97-107.

133. Heejrdt PF(van), Sluiter JW, Balazuc J. 1959. Suite des recherches sur les Chiroptères dans les grottes de l'ArdècheCampagnes de 1957-1958. Bulletin Mensuel de la Société Linnéenne de Lyon, 28(6), 165-169.

134. Hemming F, International Commission on Zoological Nomenclature. 1957. Direction 66. Opinions and declarations rendered by the International Commission on Zoological Nomenclature, volume 1, section E, partie E, 6. pp. 87-110

135. Hermann JF. 1804. Mémoire aptérologique. Imprimerie F.G. Levrault : Strasbourg. 144 p. +9 plates.

136. Herriot F. 1965. La Grotte des Excentriques, topographie et faune. Bulletin de la Société d'Histoire Naturelle de Moselle, 39, 153-172. 
137. Herriot F, Henry J-P. 1960. Contribution à l'étude de la faune cavernicole de Lorraine. Diplôme d'Études Supérieures. Sciences Naturelles, Nancy, 20 février, 1960, 95 p.

138. Hirst S. 1923. On some new or little-known species of Acari. Proceedings of the Zoological Society of London, 1923(2), 971-1000.

139. Hirst S. 1927. Note on Acari, mainly belonging to the Genus Spinturnix von Heyden. Proceedings of the Zoological Society of London, 97(2), 323-338.

140. Hopkins GHE. 1949. The host-associations of the lice of mammals. Proceedings of the Zoological Society of London, 119(2), 387-604.

141. Hopkins GHE. 1957. Host-associations of Siphonaptera, in Premier Symposium sur la spécificité parasitaire des parasites de Vertébrés, avril 1957, Neuchâtel (Suisse). Publications de l'Union Internationale des Sciences biologiques, série B, 32 . 324 p. (pp. 64-87).

142. Hopkins GHE, Rothschild M. 1956. An illustrated Catalogue of the Rothschild Collection of Fleas (Siphonaptera) in the British Museum (Natural History). With keys and short descriptions for the identifications of families, genera, species and subspecies of the Order. Volume II. Coptopsyllidae, Vermipsyllidae, Stephanocircidae, Ischnopsyllidae, Hypsophthalmidae and Xiphiopsyllidae. Trustees of the British Museum: London, 446 p. +32 plates.

143. Hornok S, Estrada-Peña A, Kontschán J, Plantard O, Kunz B, Mihalca AD, Thabah A, Tomanović S, Burazerović J, Takács N, Görföl T, Estók P, Vuong Tan T, Szóke K, Fernández de Mera IG, de la Fuente J, Takahashi M, Yamauchi T, Takano A. 2016. High degree of mitochondrial gene heterogeneity in the bat tick species Ixodes vespertilionis, I. ariadnae and I. simplex from Eurasia. Parasites \& Vectors, 8, 457.

144. Horvat Ž, Čabrilo B, Paunović M, Karapandža B, Jovanović J, Budinski I, Bjelić Čabrilo O. 2015. The helminth fauna of the greater horseshoe bat (Rhinolophus ferrumequinum) (Chiroptera: Rhinolophidae) on the territory of Serbia. Biologia Serbica, 37(1-2), 64-67.

145. Horvat Ž, Čabrilo B, Paunović M, Karapandža B, Jovanović J, Budinski I, Bjelić Čabrilo O. 2017. Gastrointestinal digeneans (Platyhelminthes: Trematoda) of horseshoe and vesper bats (Chiroptera: Rhinolophidae and Vespertilionidae) in Serbia. Helminthologia, 54(1), 17-25.

146. Hůrka K. 1963. Bat fleas of Czekoslovakia. Acta Universitatis Carolinae Biologica, 1, 1-73.

147. Hůrka K. 1964. Distribution, bionomy and ecology of the European bat flies with special regard to the Czechoslovak fauna (Dip., Nycteribiidae). Acta Universitatis Carolinae Biologica, 1964(3), 167-234.

148. Hůrka K. 1970. Revision der Nycteribiidae und StreblidaeNycteriboscinae aus der Dipterensammlung des Zoologischen Museum in Berlin, II. Mit Beschreibung von Basilia (Basilia) mediterranea n. sp. Mitteilungen aus dem Zoologischen Museum in Berlin, 46, 239-246.

149. Hůrka K. 1984. New taxa and new records of Palearctic Nycteribiidae and Streblidae (Diptera: Pupipara). Věstník Československé Společnosti Zoologické, 48, 90-101.

150. Hůrka K, Soós A. 1986. Family Nycteribiidae, in Catalogue of Palearctic Diptera. Volume 11: Scathophagidae - Hypodermatidae. Soós A, Editor. Papp L (Assistant Editor). Elsevier: Amsterdam, Oxford, New-York and Tokyo. 346 p. (pp. 226-234).

151. Husson R. 1947. Diptères des galeries de mines de France. Notes Biospéologiques, 1, 37-52.

152. Husson R, Daum J. 1957. Beitrag zur Chiropterenfauna alter Bergwerksstollen und künstlicher Höhlen im Saarland, in Lothringen und im französischen Jura. Annales Universitatis Saraviensis-Scienta, 1957, 74-81.
153. Inventaire National du Patrimoine Naturel of the Muséum National d'Histoire Naturelle. https://inpn.mnhn.fr/accueil/index.

154. Jeannel R. 1926. Faune cavernicole de la France. Avec une étude des conditions d'existence dans le domaine souterrain. Collection Encyclopédie Entomologique, Série A, Travaux généraux, number 7. Paul Lechevalier Éditeur : Paris. 334 p.

155. Jobling FRES. 1934. A revision of the genus Nycteribosca Speiser (Diptera Pupipara, Streblidae). Parasitology, 26(1), 64-97.

156. Jolivet P. 1946. Captures de puces et de diptères. L'Entomologiste, 2(4), 160.

157. Jordan K. 1942. On four new palearctic bat-fleas in the British Museum collection. Eos, 18, 243-250.

158. Joyeux C, Baer J-G. 1934. Sur quelques Cestodes de France. Archives du Muséum National d'Histoire Naturelle, $6^{\text {ème }}$ série, 11, 157-171.

159. Joyeux C, Baer J-G. 1936. Les Cestodes. Collection Faune de France, $n^{\circ} 30$, Paul Lechevalier et fils : Paris. 613 p.

160. Kieffer J-J. 1908. Quatrième contribution à la faune et la flore de Bitche. Bulletin de la Société d'Histoire Naturelle de Metz, $25^{\text {ème }}$ cahier, $3^{\text {ème }}$ série, tome $1,9-45$.

161. Kolebinova M, Vercammen-Grandjean P-H. 1970. Neotrombicula vandeli et Riedlinia petarberoni, deux Trombiculidae larvaires nouveaux et aveugles, Acariens parasites d'une chauve-souris. Annales de Spéléologie, 25(1), 173-178.

162. Kolebinova MG. 1970. Larves des Trombiculidae (Acarina) de la Corse, des Pyrénées et de la Crète. Bulletin de l'Institut de Zoologie et Musée de Sofia, 32, 93-105.

163. Kolenati FA. 1857. Die Parasiten der Chiroptern. Verlagsbuchhandlung von Rudolf : Kuntze, Dresden. 15 p.

164. König C, König I. 1961. Zur Ökologie und Systematik südfranzösischer Fledermäuse. Bonner Zoologische Beiträge: Herausgeber: Zoologisches Forschungsinstitut und Museum Alexander Koenig, 12(3-4), 189-230.

165. Krištofík J, Danko Š. 2012. Arthropod ectoparasites (Acarina, Heteroptera, Diptera, Siphonaptera) of bats in Slovakia. Vespertilio, 16, 167-189.

166. Lamontellerie M. 1965. Les Tiques (Acarina, Ixodoidea) du Sud-Ouest de la France. Annales de Parasitologie Humaine et Comparée, 40(1), 87-100.

167. Lanza B. 1999. I parassiti dei pipistrelli (Mammalia, Chiroptera) della fauna Italiana. Monografie - Museo Regionale di Scienze Naturali, Torino, $\mathrm{n}^{\circ} 30,318 \mathrm{p}$.

168. Latreille P-A. 1797. Précis des caractères génériques des insectes, disposés dans un ordre naturel. Imprimerie de F. Bourdeaux : Brive. $201 \mathrm{p}$.

169. Latreille P-A. 1803-1804. Histoire naturelle, générale et particulière des crustacés et des insectes, Tome VIII. Imprimerie F. Dufart : Paris. 411 p.

170. Latreille P-A. 1804-1805. Histoire naturelle, générale et particulière des crustacés et des insectes, Tome XIV. Imprimerie F. Dufart : Paris. 432 p.

171. Latreille P-A. 1806. Genera crustaceorum et insectorum: secundum ordinem naturalem in familias disposita, iconibus exemplisque plurimis explicata. Tome 1. Apud Amand Konig, Bibliopolam : Parisiis et Argentorati [=Paris and Strasbourg]. $302 \mathrm{p}$.

172. Latreille P-A. 1817. Le règne animal distribué d'après son organisation: pour servir de base a l'Histoire naturelle des animaux et d'introduction a l'anatomie comparée, Tome III, contenant les Crustacés, les Arachnides, les Insectes, Chez Deterville libraire : Paris. 540 p.

173. Latreille P-A. 1818. Nyctères, in Collectif, Nouveau dictionnaire d'Histoire naturelle, appliquée aux arts, à l'agriculture, à l'économie rurale et domestique, à la médecine, etc. Tome 
XXIII. [Words NIL to ORC]. Chez Deterville libraire : Paris. 612 p. (pp. 130-134).

174. Lavier G. 1924. Eimeria hessei n. sp., coccidie intestinale de Rhinolophus hipposideros. Annales de Parasitologie Humaine et Comparée, 2(4), 335-339.

175. Leach WE. 1817. Descriptions of three species of the genus Phthiridium of Hermann. The zoological miscellany: being descriptions of new, or interesting animals, 3, 54-56 + one plate.

176. Leclerq M, Théodoridès J. 1950. Some ectoparasites of birds and mammals observed recently in France. The Entomologist's Monthly Magazine, 86, 74.

177. Léger C, Pierre C. 2018. Les parasites métazoaires des Chiroptères : état des lieux (1862-2017) en Lorraine et apport de données pour la Moselle et le Haut-Rhin. Bulletin de la Société d'Histoire Naturelle de la Moselle, 54, 119-136.

178. Lehmuller D. 1967. Baguage de Chiroptères dans quelques grottes lorraines. Union spéléologique autonome de Nancy (USAN) Report: Nancy. 19 p.

179. Leugé F. 1991. Des captures aux filets, pour quoi faire ? in: Actes des troisièmes Rencontres nationales « Chauves-souris », 22 et 23 avril 1989, Malesherbes (Loiret) organisées par Objectif nature, Dammarie-les-Lys et la SFÉPM, Nort-sur-Erdre. Société Française pour l'Étude et la Protection des Mammifères (SFÉPM) editor : Nort-sur-Erdre. 134 p. (pp. 13-17).

180. López-Neyra CR. 1942. Revisión del género Hymenolepis Weinland (Continuación). Revista Ibérica de Parasitología, 2(2), 113-256.

181. Lord JS, Brooks DR. 2014. Bat Endoparasites: A UK Perspective, in Bats (Chiroptera) as Vectors of Diseases and Parasites. Klimpel S, Mehlhorn H, Editors. Springer: Berlin. 187 p. (pp. 63-86).

182. Lord JS, Parker S, Parker F, Brooks DR. 2012. Gastrointestinal helminths of pipistrelle bats (Pipistrellus pipistrellus/Pipistrellus pygmaeus) (Chiroptera: Vespertilionidae) of England. Parasitology, 139, 366-374.

183. Lucas PH. 1840. Histoire naturelle des crustacés, des arachnides et des myriapodes. P. Duménil éditeur : Paris. 600 p.

184. Lutz HL, Hochachka WM, Engel JI, Bell JA, Tkach VV, Bates JM, Hackett SJ, Weckstein JD. 2015. Parasite prevalence corresponds to host life history in a diverse assemblage of Afrotropical birds and haemosporidian parasites. PloS One, 10(4), e0121254.

185. Maa TC. 1971. An annotated bibliography of batflies (Diptera: Streblidae; Nycteribiidae). Pacific Insects Monograph, 28, 119-211.

186. Manéval H. 1928. La grotte de la Denise, près Le Puy. Capture intéressante de deux Nycteribiides sur des chauves-souris. Bulletin Bi-Mensuel de la Société Linnéenne de Lyon, 7(18), 148-149.

187. Masson D. 1989. Sur l'infestation de Myotis nattereri (Kuhl, 1818) (Chiroptera, Vespertilionidae) par Basilia nattereri (Kolenati, 1857) (Diptera, Nycteribiidae) dans le sud-ouest de la France. Annales de Parasitologie Humaine et Comparée, 64(1), 64-71.

188. Matskási I. 1967. The systematico-faunistical survey of the trematode fauna of Hungarian bats I. Annales HistoricoNaturales Musei Nationalis Hungarici, 59, 217-238.

189. Médard P, Guiguen C, Beaucournu J-C. 1997. Nouvelles récoltes d'Argas transgariepinus White, 1846 tique de chiroptères (Acarina - Ixodoidea - Argasidae) en France et au Maroc. Travaux Scientifiques du Parc national de Port Cros, 18, 31-35.

190. Mégnin P. 1876. Mémoire sur l'organisation et la distribution zoologique des acariens de la famille des Gamasides. Journal de l'Anatomie et de la Physiologie Normales et Pathologiques de l'Homme et des Animaux, 12, 288-336.
191. Mégnin P. 1892. Les acariens parasites. Gauthier-Villars et fils and G. Masson éditeurs : Paris. $182 \mathrm{p}$.

192. Melaun C, Werblow A, Busch MW, Liston A, Klimpel S. 2014. Bats as potential reservoir hosts for vector-borne diseases, in Bats (Chiroptera) as Vectors of Diseases and Parasites. Klimpel S, Mehlhorn H, Editors. Springer: Berlin. $187 \mathrm{p}$.

193. Melville RV, Smith JDD. 1987. Official lists and indexes of names and works in zoology. The International Trust for Zoological Nomenclature et The International Commission on Zoological Nomenclature: London. 366 p.

194. Mészáros F. 1967. Seuratum mucronatum (Rud. 1808) aus Fledermäusen in Ungarn. Annales Historico-Naturales Musei Nationalis Hungarici, 59, 239-242.

195. Micherdziński W. 1980. Eine taxonomische analyse der familie Macronyssidae Oudemans, 1936. I. Subfamilie Ornithonyssinae Lange, 1958 (Acarina, Mesostigmata). Collection Praca wykonana w ramach problemu MR, number II. 3. Państwowe Wydawnictwo Naukowe: Warszawa (Warsaw) and Kraków (Krakow). 264 p.

196. Murai É. 1976. Cestodes of bats in Hungary. Parasitologia Hungarica, 9, 41-62.

197. Neumann LG. 1899. Révision de la famille des Ixodidés ( $3^{\text {ème }}$ mémoire). Mémoires de la Société Zoologique de France, 12(1), 107-294.

198. Neumann LG. 1910. Sur trois types d'Ixodinae de Kolenati appartenant au Muséum d'Histoire naturelle de Paris. Bulletin du Muséum National d'Histoire Naturelle, 4, 191-193.

199. Neumann LG. 1915-1916. Ixodidei (Acariens) première série. Biospeologica XXXVII. Archives de Zoologie Expérimentale et Générale, 55(12), 515-527 + plate X.

200. Neveu-Lemaire M, Joyeux C, Larrousse F, Isobe $M$, Lavier G. 1924. XXXII. Parasites de chauves-souris de la Côte-d'Or. Comptes-rendus du Congrès des Sociétés Savantes de Paris et des Départements. Section des Sciences, 1924, 274-280.

201. Nuttall GHF, Warburton C, Cooper WF, Robinson LE. 1911. Ticks. A monograph of the Ixodoidea. Part II. Cambridge University Press: Cambridge. 348 p.

202. Odening K. 1971. Das Exkretionssystem von Cephalogonimus retusus und Mesotretes peregrinus (Trematoda). Helminthologia, 10, 121-127.

203. Olivier E. 1882. Faune du Doubs, ou Catalogue raisonné des animaux sauvages (Mammifères, Reptiles, Batraciens, Poissons) observés jusqu'à ce jour dans ce département. Mémoires de la Société d'Émulation du Doubs, 5(7), 73-139.

204. Olivier E. 1904. Faune de l'Allier. Insectes - ordre des Diptères. Sous-ordre des Pupipares. Revue Scientifique du Bourbonnais et du Centre de la France, 17, 77-81.

205. Oudemans AC. 1902. Notes on Acari, fourth series. Tijdschrift der Nederlandsche Dierkundige Vereeniging, $2^{\text {nd }}$ serie, 2(7), 276-311.

206. Penot A. 1831. Statistique générale du département du Haut-Rhin publiée par la Société industrielle de Mulhausen. Imprimerie de Jean Risler et Compagnie : Mulhausen [=Mulhouse]. $482 \mathrm{p}$.

207. Péricart J. 1972. Hémiptères Anthocoridae, Cimicidae et Microphysidae de l'Ouest-Paléarctique. Collection Faune de l'Europe et du Bassin méditerranéen, number 7. Masson et Compagnie and Centre National de la Recherche Scientifique (CNRS) : Paris. $406 \mathrm{p}$

208. Pinichpongse S. 1963. A review of the Chirodiscinae with descriptions of new taxa (Acarina, Listrophoridae) (Part two). Acarologia, 5(2), 266-278. 
209. Pocora I, Ševčík M, Uhrin M, Bashta AT, Pocora V. 2013. Morphometric notes and nymphal stages description of mite species from the Spinturnix myoti group (Mesostigmata: Spinturnicidae) from Romania and Ukraine. International Journal of Acarology, 39(2), 153-159.

210. Prasad V. 1969. Bat Mites (Acarina: Spinturnicidae) mainly from south-east Asia and the Pacific Region. Acarologia, 11(4), 657-677.

211. Quentin JC. 1969. Essai de classification des Nématodes Rictulaires. Mémoires du Muséum National d'Histoire Naturelle, nouvelle série, série A. Zoologie, 54(2), 55-115.

212. Radovsky FJ. 1967. The Macronyssidae and Laelapidae (Acarina: Mesostigmata) parasitic on bats. University of California Publications in Entomology, 46, 1-288.

213. Rageau J. 1972. Répartition géographique et rôle pathogène des tiques (Acariens: Argasidae et Ixodidae) en France. Wladomości Parazytologiczne, 18(4-6), 707-719.

214. Rémy P. 1927. Sur la faune des grottes de Sainte-Reine, près de Pierre-la-Treiche (Meurthe-et-Moselle). Bulletin BiMensuel de la Société Linnéenne de Lyon, 6(15), 118-120.

215. Rémy P. 1932. Contribution à l'étude de la faune cavernicole en Lorraine. Les Grottes de Sainte Reine. Bulletin de la Société d'Histoire Naturelle de la Moselle, 33, 55-71.

216. Rémy P. 1948. Installation d'un Anoploure sur une chauvesouris. La Feuille des Naturalistes, 3(9-10), 97.

217. Rollinat R, Trouessart ÉL. 1896. Sur la reproduction des chauves-souris. Le Vespertilion murin. Mémoires de la Société Zoologique de France, 9, 214-240.

218. Rollinat R, Trouessart ÉL. 1897. Sur la reproduction des chauves-souris. II. Les Rhinolophes et Note sur leurs parasites épizoïques. Mémoires de la Société Zoologique de France, 10, 114-138.

219. Rothschild C. 1910. Liste des Siphonaptera du Muséum d'Histoire Naturelle de Paris, accompagnée de descriptions d'espèces nouvelles. Annales des Sciences Naturelles, Zoologie, 12, 203-216.

220. Rousseau E. 1839. Mémoire zoologique et anatomique sur la Chauve-souris commune dite Murin, ayant principalement rapport à la première et à la seconde dentition de ce Chéiroptère. Magasin de Zoologie, d'Anatomie Comparée et de Paléontologie, troisième livraison, 1839 , première section. $1-47+9$ plates.

221. Roy L, Chauve C. 2007. Historical review of the genus Dermanyssus Dugès, 1834 (Acari: Mesostigmata: Dermanyssidae). Parasite, 14, 87-100.

222. Roy L, Chauve C. 2009. The genus Dermanyssus (Mesostigmata: Dermanyssidae): History and species characterization, in Trends in Acarology: Proceedings of the 12th International Congress Held from 21-26 August 2006 in Amsterdam. Sabelis MW, Bruin J, Editors. Springer: Dordrecht. 566 p.

223. Rudnick A. 1960. A revision of the mites of the family of Spinturnicidae (Acarina). University of California Publications in Entomology, 17(2), 157-284.

224. Rupp D, Zahn A, Ludwig P. 2004. Actual records of bats ectoparasites in Bavaria (Germany). Spixiana, 27(2), 185-190.

225. Sachanowicz K, Ciechanowski M, Piksa K. 2017. Spinturnix dasycnemi (Acari: Spinturnicidae) - a poorly known Palaearctic bat mite: first records in Poland and morphometric separation from two other species of the myoti group. Annals of Parasitology, 63(1), 49-56.

226. Schaik J (van), Kerth G, Bruyndonckx N, Christe P. 2014. The effect of host social system on parasite population genetic structure: comparative population genetics of two ectoparasitic mites and their bat hosts. BMC Evolutionary Biology, 14, 18.
227. Séguy E. 1938. Les Puces de la région de Fontainebleau et de la Vallée du Loing [Aphinaptera]. Travaux des Naturalistes de la Vallée du Loing, 9, 5-36.

228. Séguy E. 1944. Insectes ectoparasites (Mallophages, Anoploures, Siphonaptères). Collection Faune de France de la Fédération Française des Sociétés de Sciences Naturelles, Office Central de Faunistique : Paris. 684 p.

229. Senevet G. 1937. Ixodidés. Collection Faune de France de la Fédération Française des Sociétés de Sciences Naturelles. Office Central de Faunistique. Paul Lechevalier et fils : Paris. 104 p.

230. Simões MB, Moreira NIB, Leite YLR. 2019. First record of Pterygodermatites (Pterygodermatites) (Nematoda: Rictulariidae) in South America, with the description of a new species from the Atlantic Forest, southeast Brazil. Zootaxa, 4629(1), 96-108.

231. Skuratowicz W. 1967. Klucze do Oznaczania owadow Polski. Czesc XXIX Pchly. Siphonaptera (Aphinaptera). Panstwowe Wydawnictwo Naukowe : Warszawa (Warsaw). 684 p.

232. Société Entomologique de France. 1895. M. le Docteur Trouessart communique (en collaboration avec M. le Professeur G. Canestrini, de Padoue) la diagnose d'une espèce nouvelle de Sarcoptide pilicole (Listrophorinae). Bulletin des Séances et Bulletin Bibliographique de la Société Entomologique de France, 1, XXXVIII-XXXIX.

233. Sonsino P. 1887-1889. Notize elmintologiche. Atti della Società Toscana di Scienze Naturali. Processi Verbali, 6, 113-131.

234. Stefka J, Hypsa V. 2008. Host specificity and genealogy of the louse Polyplax serrata on field mice, Apodemus species: a case of parasite duplication or colonization? International Journal for Parasitology, 38(6), 731-741.

235. Stiles CW, Nolan MO. 1931. Key catalogue of parasites reported for chiroptera (bats) with their Possible Public Health Importance. National Institute of Health Bulletin (December 1930), 155, 603-742.

236. Streito J-C. 2003. Collaboration. Punaises et chiroptères. L'Envol des Chiros, 7, 15.

237. Szentiványi T, Estók P, Földvári M. 2016. Checklist of host associations of European bat flies (Diptera: Nycteribiidae, Streblidae). Zootaxa, 4205(2), 101-126.

238. Szentivanyi T, Glaizot O, Christe P. 2019. Bat flies and their microparasites: current knowledge and distribution. Frontiers in Veterinary Science, 6, 115.

239. Tétry A. 1938. Contribution à l'étude de la faune de l'Est de la France (Lorraine). Thèse de Sciences, Imprimerie Georges Thomas: Nancy. 453 p.

240. Theodor O. 1954. [Part 66a.] Nycteribiidae, in: Lindner E. Die Fliegen der palaearktischen Region. Lieferung 174. E. Schweizerbart'sche Verlagsbuchhandlung (Erwin Nägele) : Stuttgart. $44 \mathrm{p}$.

241. Theodor O, Moscona A. 1954. On the bat parasites in Palestine I. Nycteribiidae, Streblidae, Hemiptera, Siphonaptera. Parasitology, 44(1-2), 157-245.

242. Théodoridès J. 1953. Première contribution à l'étude des ectoparasites de vertébrés des Pyrénées-Orientales. Vie et Milieu, 4(4), 753-756.

243. Timon-David J. 1964. Contribution à la connaissance des helminthes du Rhinolophe fer à cheval en Provence. Vie et Milieu, 15, 139-151.

244. Tiraboschi C. 1904. Les rats, les souris et leurs parasites cutanés dans leurs rapports avec la propagation de la peste bubonique. Archives de Parasitologie, 8(2), 161-349.

245. Tkach V, Swiderski Z. 1996. Scanning electron microscopy of the rare nematode species Pterygodermatites bovieri (Nematode, Rictatuliriidae) parasite of bats. Folia Parasitologica, 34, 301-304. 
246. Trägårdh I. 1911-1912. Acari (1 $1^{\text {ère }}$ série). Biospeologica XXII (avec pl. XVIII à XXIV). Archives de Zoologie et Générale, cinquième série, 8(7), 519-620.

247. Trouessart ÉL. 1880. Note sur deux espèces de ChauvesSouris nouvelles pour la faune de Maine-et-Loire. Bulletin de la Société d'Études Scientifiques d'Angers, 8-9, 206-207.

248. Trouessart ÉL. 1880. Deuxième note sur une troisième et quatrième espèce de Chauves-Souris nouvelles pour la faune de Maine-et-Loire, et sur deux espèces de parasites épizoaires, absolument nouvelles, trouvées sur ces Cheiroptères. Bulletin de la Société d'Études Scientifiques d'Angers, 8-9, 225-227.

249. Trouessart ÉL. 1895. Sur les métamorphoses du genre Myobia et diagnoses d'espèces nouvelles d'Acariens. Bulletin de la Société Entomologique de France, 1895, CCXIII-CCXVI.

250. Trouessart ÉL. 1896. Sur deux espèces et un genre pluriel nouveaux de Sarcoptides psoriques. Comptes Rendus des Séances de la Société de Biologie et de ses filiales, 10th serie, $3,747-749$.

251. Uchikawa K. 1989. Ten new taxa of chiropteran myobiids of the genus Pteracarus (Acarina, Myobiidae). Bulletin of the British Museum (Natural History). Zoology, 55(1), 97-108.

252. Uchikawa K, Dusbábek F. 1978. Studies on mesostigmatid mites parasitic on mammals and birds in Japan. VIII. Bat mites of the genus Eyndhovenia Rudnick, 1960, with redescription of Eyndhovenia euryalis euryalis (Canestrini, 1884). Bulletin of the National Science Museum. Series A (Zoology), 4(4), 245-261.

253. Usinger RL. 1966. Monograph of Cimicidae: (Hemiptera, Heteroptera). Collection. The Thomas Say Foundation, number 7, Entomological Society of America: Calvert Road (Maryland, USA). 596 p.
254. Usinger RL, Beaucournu J-C. 1967. Sur deux Cimex (Insecta, Heteroptera), nouveaux pour la faune française, parasites des chauves-souris. Annales de Parasitologie Humaine et Comparée, 42(2), 69-71.

255. Vaucher C. 1992. Revision of the genus Vampirolepis Spasskij, 1954 (Cestoda: Hymenolepididae). Memórias do Instituto Oswaldo Cruz, 87 (suppl. I), 299-304.

256. Vitzthum HG. 1932. Acarinen aus dem Karst (excl Oribatei). Zoologische Jahrbücher: Abteilung für Systematik, Ökologie und Geographie der Tiere, 63(5-6), 681-700.

257. Walckenaer CA. 1802. Faune parisienne, insectes ou Histoire abrégée des insectes des environs de Paris, classés d'après le système de Fabricius. Tome second. Dentu Imprimeur-Libraire : Paris. 438 p.

258. Walckenaer CA, Gervais P. 1844. Histoire naturelle des insectes. Tome 3. Aptères. Librairie Encyclopédique de Roret : Paris. $476 \mathrm{p}$.

259. Walckenaer CA, Gervais P. 1847. Histoire naturelle des insectes aptères. Tome 4. Librairie Encyclopédique de Roret : Paris. 476 p.

260. Westwood JO. 1834. On Nycteribia, a genus of wingles insects. Proceedings of the Zoological Society of London, 2-3, 135-140.

261. Wu Z, Yang L, Ren X, He G, Zhang J, Yang J, Qian Z, Dong J, Sun L, Zhu Y, Du J, Yang F, Zhang S, Jin Q. 2016. Deciphering the bat virome catalog to better understand the ecological diversity of bat viruses and the bat origin of emerging infectious diseases. ISME Journal, 10(3), 609-620.

262. Yamaguti S. 1959. Systema Helminthum. Volume II: the Cestodes of Vertebrates. Interscience Publishers: New-York and London. 860 p.

Cite this article as: Léger C. 2020. Bat parasites (Acari, Anoplura, Cestoda, Diptera, Hemiptera, Nematoda, Siphonaptera, Trematoda) in France (1762-2018): a literature review and contribution to a checklist. Parasite 27, 61.

\section{PARASTE}

An international open-access, peer-reviewed, online journal publishing high quality papers on all aspects of human and animal parasitology

Reviews, articles and short notes may be submitted. Fields include, but are not limited to: general, medical and veterinary parasitology; morphology, including ultrastructure; parasite systematics, including entomology, acarology, helminthology and protistology, and molecular analyses; molecular biology and biochemistry; immunology of parasitic diseases; host-parasite relationships; ecology and life history of parasites; epidemiology; therapeutics; new diagnostic tools.

All papers in Parasite are published in English. Manuscripts should have a broad interest and must not have been published or submitted elsewhere. No limit is imposed on the length of manuscripts.

Parasite (open-access) continues Parasite (print and online editions, 1994-2012) and Annales de Parasitologie Humaine et Comparée (1923-1993) and is the official journal of the Société Française de Parasitologie. 